\title{
Statistical Inferences on Uniform Distributions: The Cases of Boundary Values Being Parameters
}

\author{
Ismail Erdem \\ Baskent University Faculty of Science and Letters Department of Statistics and Computer Science Baglica, Ankara, 06530, Turkey
}

\begin{abstract}
If a continuous random variable $X$ is uniformly distributed over the interval and if any of the two boundary values is unknown, it is necessary to make inferences related to the unknown parameter. In this work, for the unknown boundary values of $\mathrm{X}$, some unbiased estimators based on certain order statistics and sample mean are suggested. These estimators are compared in terms their efficiencies. The most efficient unbiased estimator is used to provide confidence intervals and tests of hypotheses procedures for the unknown parameter (the unknown boundary value).
\end{abstract}

Keywords Uniform Distribution, Order Statistics, Unbiased Estimators, Efficiency, Confidence Intervals, Tests of Hypotheses

\section{Background}

The books and articles, listed in the reference list of this study with the reference numbers from[1] to[18], and many more not in the list, studied order statistics in such a way that every aspects of the topic has already been well explored.

However, there is no inferential study, as far as I am aware of, on the boundary values of the uniform distributions. This work aims at the determination of good estimators for the boundary values of uniform distributions. Based on the determined good estimator, construction of confidence intervals and procedures for the test of hypotheses are established.

For illustration, a simulation study is conducted and summaries of the simulation study are provided. The raw data and computations are provided in the appendix of this paper.

\section{Introduction}

A uniformly distributed continuous random variable, assuming real values in the interval $\left(\theta_{1}, \theta_{2}\right)$, has the following probability density function (pdf)

$$
f(x)=\frac{1}{\theta_{2}-\theta_{1}}, \theta_{1}<x<\theta_{2}
$$

For this distribution, the Maximum Likelihood Estimators

\footnotetext{
* Corresponding author:

iserdem@baskent.edu.tr (Ismail Erdem)

Published online at http://journal.sapub.org/ijps

Copyright $@ 2012$ Scientific \& Academic Publishing. All Rights Reserved
}

(MLE) of $\theta_{1}$ and $\theta_{2}$ will be $Y_{1}$ and $Y_{n}$, the smallest and the largest order statistics, respectfully.

$\theta_{1}$ and $\theta_{2}$ need to be estimated for that each moment of the random variable $\mathrm{X}$ is, as shown below, a function of these parameters.

$$
E\left(X^{k}\right)=\frac{1}{\theta_{2}-\theta_{1}} \int_{\theta 1}^{\theta 2} x^{k} d x=\frac{\left(\theta_{2}^{k+1}-\theta_{1}^{k+1}\right)}{(k+1)\left(\theta_{2}-\theta_{1}\right)}, k=1,2,3, \ldots
$$

Let $X_{1}, X_{2}, \ldots, X_{n}$ be a random sample of size $\mathrm{n}$ from a uniform distribution over the interval $\left(\theta_{1}, \theta_{2}\right)$, and let $Y_{i}=$ ith order statistic, $i=1,2, \ldots, n$.

The pdf of ith order statistic is obtained by the following general formula, as given in almost all mathematical statistics textbooks, like the ones with the reference numbers[1],[2],[3],[4],[6], and[7].

$$
f_{Y i}(y)=\frac{n !}{(i-1) !(n-i) !}[F(y)]^{(i-1)}[1-F(y)]^{(n-i)} f_{Y}(y)(3)
$$

Where, $\mathrm{n}=$ size of the random sample, $F(y)$ is the cumulative distribution function (cdf) of the distribution, and $f_{Y_{i}}(y)$ is the pdf of the random variable (ith order statistic) $Y_{i}$.

Specifically, if the pdf given in (1) is used we obtain the following cdf.

$$
F(y)=\int_{\theta 1}^{y} \frac{1}{\theta_{2}-\theta_{1}} d x=\frac{y-\theta_{1}}{\theta_{2}-\theta_{1}}, \theta_{1}<y<\theta_{2}
$$

Using the equations (3) and (4), we obtain the following pdfs for $Y_{1}$ and $Y_{n}$. 


$$
\begin{aligned}
& f_{Y_{1}}(y)=n\left[1-\frac{y_{1}-\theta_{1}}{\theta_{2}-\theta_{1}}\right]^{(n-1)}\left(\frac{1}{\theta_{2}-\theta_{1}}\right)=\frac{n}{\left(\theta_{2}-\theta_{1}\right)^{n}}\left(\theta_{2}-y_{1}\right)^{(n-1)}, \theta_{1}<y_{1}<\theta_{2} \\
& f_{Y_{n}}\left(y_{n}\right)=n\left[\frac{y_{n}-\theta_{1}}{\theta_{2}-\theta_{1}}\right]^{(n-1)}\left(\frac{1}{\theta_{2}-\theta_{1}}\right)=\frac{n}{\left(\theta_{2}-\theta_{1}\right)^{n}}\left(y_{n}-\theta_{1}\right)^{(n-1)}, \theta_{1}<y_{n}<\theta_{2}
\end{aligned}
$$

Use of the above pdfs will enable us to obtain the expected values and the variances of $Y_{1}$, and $Y_{n}$.

To take advantage of the computational simplicity lets introduce the following transformations.

$$
\begin{aligned}
& \text { Let } U_{1}=\theta_{2}-Y_{1} \rightarrow f_{U_{1}}\left(u_{1}\right)=\frac{n}{\left(\theta_{2}-\theta_{1}\right)^{n}} u_{1}^{n-1}, 0<u_{1}<\left(\theta_{2}-\theta_{1}\right), \\
& \text { and let } \\
& U_{n}=Y_{n}-\theta_{1} \rightarrow f_{U_{n}}\left(u_{n}\right)=\frac{n}{\left(\theta_{2}-\theta_{1}\right)^{n}} u_{n}^{n-1}, 0<u_{n}<\left(\theta_{2}-\theta_{1}\right) \\
& E\left(U_{1}\right)=\int_{0}^{\theta_{2}-\theta_{1}} u_{1} \frac{n}{\left(\theta_{2}-\theta_{1}\right)^{n}} u_{1}^{n-1}=\frac{n}{\left(\theta_{2}-\theta_{1}\right)^{n}}\left(\frac{u^{n+1}}{(n+1)}\right)_{u=0}^{\left.\theta_{2}-\theta_{1}\right)}=\frac{n\left(\theta_{2}-\theta_{1}\right)}{(n+1)} \\
& E\left(U_{1}^{2}\right)=\int_{0}^{\theta_{2}-\theta_{1}} u_{1}^{2} \frac{n}{\left(\theta_{2}-\theta_{1}\right)^{n}} u_{1}^{n-1}=\frac{n}{\left(\theta_{2}-\theta_{1}\right)^{n}}\left(\frac{u^{n+2}}{(n+2)}\right)_{u=0}^{\left(\theta_{2}-\theta_{1}\right)}=\frac{n\left(\theta_{2}-\theta_{1}\right)^{2}}{(n+2)} \\
& \operatorname{Var}\left(U_{1}\right)=\frac{n\left(\theta_{2}-\theta_{1}\right)^{2}}{(n+2)}-\frac{n^{2}\left(\theta_{2}-\theta_{1}\right)^{2}}{(n+1)^{2}}=\frac{n\left(\theta_{2}-\theta_{1}\right)^{2}}{(n+1)^{2}(n+2)}
\end{aligned}
$$

From (7) we get $Y_{1}=\theta_{2}-U_{1}$. It then follows that

$$
\begin{aligned}
& E\left(Y_{1}\right)=\theta_{2}-E\left(U_{1}\right)=\theta_{2}-\frac{n\left(\theta_{2}-\theta_{1}\right)}{(n+1)}=\frac{n \theta_{1}+\theta_{2}}{(n+1)} \\
& \operatorname{Var}\left(Y_{1}\right)=\operatorname{Var}\left(U_{1}\right)=\frac{n\left(\theta_{2}-\theta_{1}\right)^{2}}{(n+1)^{2}(n+2)}
\end{aligned}
$$

From (7) we observe that the pdfs of $U_{1}$ and $U_{n}$ are exactly the same.

Hence,

$$
\begin{gathered}
E\left(U_{n}\right)=\frac{n\left(\theta_{2}-\theta_{1}\right)}{(n+1)}, \text { and } \\
\operatorname{Var}\left(U_{n}\right)=\frac{n\left(\theta_{2}-\theta_{1}\right)^{2}}{(n+1)^{2}(n+2)}
\end{gathered}
$$

3. Statistical Inferences Related to the

Parameter of $X \sim \operatorname{Uniform}\left(\theta_{1}, b\right)$

\section{Distribution}

From (7) we get $Y_{n}=U_{n}+\theta_{1}$. It then follows that

$$
\begin{aligned}
& E\left(Y_{n}\right)=\theta_{1}+E\left(U_{n}\right) \\
& =\theta_{1}+\frac{n\left(\theta_{2}-\theta_{1}\right)}{(n+1)}=\frac{n \theta_{2}+\theta_{1}}{(n+1)} \\
& \operatorname{Var}\left(Y_{n}\right)=\operatorname{Var}\left(U_{n}\right) \\
& =\frac{n\left(\theta_{2}-\theta_{1}\right)^{2}}{(n+1)^{2}(n+2)}
\end{aligned}
$$

Here we established the distributions, expected values, and variances of the smallest (the first) and the largest (the last) ordered statistics that are to be used in subsequent sections of this study.

\subsection{Estimation of the Parameter $\theta_{1}$ of the Distribution $X \sim \operatorname{Uniform}\left(\theta_{1}, b\right)$ by the First Order Statistic $Y_{1}$}

A uniformly distributed continuous random variable $\mathrm{X}$, over the interval $\left(\theta_{1}, b\right)$, where $\mathrm{b}$ is given constant, has the following pdf

$$
f(x)=\frac{1}{b-\theta_{1}}, \theta_{1}<x<b
$$


The MLE of $\theta_{1}$ of $X \sim \operatorname{Uniform}\left(\theta_{1}, b\right)$ will be $Y_{1}$ and its expected value and variance are from Equations given in (11),

$$
\begin{aligned}
& E\left(Y_{1}\right)=\frac{n \theta_{1}+\theta_{2}}{(n+1)}=\frac{n \theta_{1}+b}{(n+1)}, \text { and } \\
& \operatorname{Var}\left(Y_{1}\right)=\frac{n\left(\theta_{2}-\theta_{1}\right)^{2}}{(n+1)^{2}(n+2)}=\frac{n\left(b-\theta_{1}\right)^{2}}{(n+1)^{2}(n+2)}
\end{aligned}
$$

An unbiased estimator of $\theta_{1}$, based on the first order statistic is

$$
\begin{gathered}
T_{1}=\frac{(n+1) Y_{1}-b}{n}, \\
\operatorname{Var}\left(T_{1}\right)=\frac{(n+1)^{2}}{n^{2}} \operatorname{Var}\left(Y_{1}\right)=\frac{\left(b-\theta_{1}\right)^{2}}{n(n+2)}
\end{gathered}
$$

\subsection{Estimation of the Parameter $\theta_{1}$ of the Distribution}

$$
X \sim \operatorname{Uniform}\left(\theta_{1}, b\right) \text { by the Last Order Statistic } Y_{n}
$$

Another estimator of $\theta_{1}$ of $X \sim \operatorname{Uniform}\left(\theta_{1}, b\right)$ will be $Y_{n}$ and its expected value and variance are from Equations given in (12),

$$
\begin{aligned}
& E\left(Y_{n}\right)=\frac{n \theta_{2}+\theta_{1}}{(n+1)}=\frac{n b+\theta_{1}}{(n+1)} \\
& \operatorname{Var}\left(Y_{n}\right)=\frac{n\left(\theta_{2}-\theta_{1}\right)^{2}}{(n+1)^{2}(n+2)}=\frac{n\left(b-\theta_{1}\right)^{2}}{(n+1)^{2}(n+2)}
\end{aligned}
$$

By the utilization of the first equation of (3.2.1) we can obtain an unbiased estimator for $\theta_{1}$ as a function of the last order statistic $Y_{n}$

$$
\begin{gathered}
T_{2}=(n+1) Y_{n}-n b \\
\operatorname{Var}\left(T_{2}\right)=(n+1)^{2} \operatorname{Var}\left(Y_{n}\right)=\frac{n\left(b-\theta_{1}\right)^{2}}{(n+2)}
\end{gathered}
$$

\subsection{Estimation of the Parameter $\theta$ of the Distribution}

$$
X \sim \operatorname{Uniform}\left(\theta_{1}, b\right) \text { by } \bar{X}
$$

If $X \sim \operatorname{Uniform}\left(\theta_{1}, b\right)$ then its pdf is $f(x)=\frac{1}{b-\theta_{1}}, \theta<x<b$. Then by equation (1), as given below:

$$
E\left(X^{k}\right)=\frac{1}{\theta_{2}-\theta_{1}} \int_{\theta 1}^{\theta 2} x^{k} d x=\frac{\left(\theta_{2}^{k+1}-\theta_{1}^{k+1}\right)}{(k+1)\left(\theta_{2}-\theta_{1}\right)}, k=1,2,3, \ldots
$$

For $\mathrm{k}=1$ and $\theta_{2}=b$

$$
E(X)=\frac{\left(b^{2}-\theta_{1}^{2}\right)}{(2)\left(b-\theta_{1}\right)}=\frac{b+\theta_{1}}{2}
$$

For $\mathrm{k}=2$ and $\theta_{2}=b$,

$$
E\left(X^{2}\right)=\frac{\left(b^{3}-\theta_{1}^{31}\right)}{(3)\left(\theta_{2}-\theta_{1}\right)}=\frac{b^{2}+b \theta_{1}+\theta_{1}^{2}}{3}
$$

From

$$
\text { (1.3.1) }
$$
and $\operatorname{Var}(X)=\sigma^{2}=E\left(X^{2}\right)-[E(X)]^{2}$ we obtain

$$
\operatorname{Var}(X)=\frac{\left(b-\theta_{1}\right)^{2}}{12} \text {. }
$$

For any distribution, if the sample mean is $\bar{X}$, for any random sample of size $\mathrm{n}$, the followings hold true.

$$
E(\bar{X})=E(X) \text {, }
$$

and

$$
\operatorname{Var}(\bar{X})=\frac{\operatorname{Var}(X)}{n}
$$

Hence, for $X \sim \operatorname{Uniform}\left(\theta_{1}, b\right)$,

$$
\begin{aligned}
& E(\bar{X})=\frac{\left(b+\theta_{1}\right)}{2} \\
& \operatorname{Var}(\bar{X})=\frac{\left(b-\theta_{1}\right)^{2}}{12 n}
\end{aligned}
$$

From (3.3.3) an unbiased estimator of $\theta_{1}$ will be

$$
T_{3}=2(\bar{X})-b, \text { and }_{\operatorname{Var}}\left(T_{3}\right)=4 \operatorname{Var}(\bar{X})=\frac{\left(b-\theta_{1}\right)^{2}}{3 n}
$$

\subsection{Estimation of the Parameter $\theta_{1}$ of the Distribution}

\section{$X \sim$ Uniform $\left(\theta_{1}, b\right)$ by the Sample Median M}

If $X \sim \operatorname{Uniform}\left(\theta_{1}, b\right)$ then $W=X-\theta_{1} \quad$ is uniformly distributed over the interval $\left(0,\left(b-\theta_{1}\right)=\eta_{1}\right)$

To take advantage of computational simplicity, the parameter $\eta_{1}$ (and in turn $\theta_{1}$ of $\mathrm{X}$ ), of the distribution $W \sim \operatorname{Uniform}\left(0, \eta_{1}\right)$, is to be estimated by the sample median.

\section{Sample Median (M):}

Let $W_{1}, W_{2}, \ldots, W_{n}$ is a random sample of size $\mathrm{n}$ from $f(w)=\frac{1}{\eta_{1}}, \quad 0<w<\eta_{1}$

The sample median $\mathrm{M}$, depending on if $\mathrm{n}$ is odd or even, is obtained as follows.

If $W_{1}, W_{2}, \ldots, W_{n}$ are ordered, in the order of their magnitude, we obtain the following order statistics. $U_{1}<U_{2}<\ldots<U_{n}$. 
Note:

If a random sample of size $\mathrm{n}$ is taken from $X \sim \operatorname{Uniform}\left(\theta_{1}, b\right)$, then the order statistics are $\left[Y_{1}, Y_{2}, \ldots, Y_{n}\right]$.

If a random sample of size $\mathrm{n}$ is taken from $W \sim \operatorname{Uniform}\left(0, \eta_{1}\right)$, then the order statistics are $\left[W_{1}, W_{2}, \ldots, W_{n}\right]=\left[\left(Y_{1}-\theta_{1}\right),\left(Y_{2}-\theta_{1}\right), \ldots\left(Y_{n}-\theta_{1}\right)\right]$

From this observation, we can see that, obtaining sample median's distribution and its mean and variance from $\left[W_{1}, W_{2}, \ldots, W_{n}\right]$ will enable us to obtain distribution of the sample median and its mean and variance of $\left[Y_{1}, Y_{2}, \ldots, Y_{n}\right]$

If $\mathrm{n}$ is odd: $M=U_{(n+1) / 2}$;

If $\mathrm{n}$ is even: $M=\frac{U_{n / 2}+U_{(n+2) / 2}}{2}$.

3.4.1. Estimation of the Parameter $\theta_{1}$ of the Distribution

$X \sim \operatorname{Uniform}\left(\theta_{1}, b\right)$ by the Sample Median $\mathrm{n}$ is

Odd

Theorem 3.4.1. If $W \sim$ Uniform $\left(0, \eta_{1}\right)$ and if an odd sized random sample is taken from this distribution then for the sample median $\mathrm{M}$,

$$
\begin{gathered}
E(M)=\frac{\eta_{1}}{2}, E\left(M^{2}\right)=\frac{\eta_{1}^{2}(n+3)}{4(n+2)} \text { and } \\
\operatorname{Var}(M)=\frac{\eta_{1}^{2}}{4(n+2)} \mathrm{dir} .
\end{gathered}
$$

Proof:

Proof will be given by induction.

$$
\text { for } \quad \mathrm{n}=1: \quad E(M)=E(W)=\frac{\eta_{1}}{2} \text {, }
$$

and

$\operatorname{Var}(M)=\operatorname{Var}(W)=\frac{\eta_{1}^{2}}{12}=\frac{\eta_{1}^{2}}{4(n+2)}$.

For $n=3$ : the sample median is $M=U_{2}$. The pdf of $M=U_{2}$ is to be obtained, by the use of (3), as follows.

$$
\begin{aligned}
& \text { Since, } \quad f(w)=\frac{1}{\eta_{1}}, 0<w<\eta_{1} \\
& F(u)=\int_{0}^{u} \frac{1}{\eta} d w=\frac{u}{\eta_{1}}, 0<u<\eta_{1} \text {, then }
\end{aligned}
$$

$$
\begin{aligned}
f_{M}(u) & =\frac{3 !}{1 ! 1 !}\left[\frac{u}{\eta_{1}}\right]^{1}\left[1-\frac{u}{\eta_{1}}\right]^{1} \frac{1}{\eta_{1}} \\
& =\frac{6}{\eta_{1}^{3}}(u)\left(\eta_{1}-u\right), 0<u<\eta_{1}
\end{aligned}
$$

$$
\begin{aligned}
E(M) & =\int_{0}^{\eta_{1}} u \frac{6}{\eta_{1}^{3}}(u)\left(\eta_{1}-u\right) d u \\
& =\frac{6}{\eta_{1}^{3}}\left[\eta_{1} \frac{u^{3}}{3}-\left.\frac{u^{4}}{4}\right|_{0} ^{\eta_{1}}\right]=\frac{6 \eta_{1}}{12}=\frac{\eta_{1}}{2},
\end{aligned}
$$

$$
\begin{aligned}
E\left(M^{2}\right) & =\int_{0}^{\eta_{1}} u^{2} \frac{6}{\eta_{1}^{3}}(u)\left(\eta_{1}-u\right) d u \\
& =\frac{6}{\eta_{1}^{3}}\left[\eta_{1} \frac{u^{4}}{4}-\frac{u^{5}}{5}\right]_{0}^{\eta_{1}}=\frac{6 \eta_{1}^{2}}{20}=\frac{\eta_{1}^{2}(n+3)}{4(n+2)} \\
\operatorname{Var}(M) & =\frac{3 \eta_{1}^{2}}{10}-\frac{\eta_{1}^{2}}{4}=\frac{\eta^{2}}{20}=\frac{\eta_{1}^{2}}{4(n+2)}
\end{aligned}
$$

Similarly, for $n=5, \quad M=U_{3}$ and from (3)

$$
\begin{gathered}
f_{M}(u)=\frac{5 !}{2 ! 2 !}\left[\frac{u}{\eta_{1}}\right]^{2}\left[1-\frac{u}{\eta_{1}}\right]^{2} \frac{1}{\eta_{1}} \\
=\frac{30}{\eta_{1}^{5}}\left(u^{2}\right)\left(\eta_{1}-u\right)^{2}, 0<u<\eta_{1} \\
E(M)=\int_{0}^{\eta_{1}} u \frac{30}{\eta_{1}{ }^{2}}\left(u^{2}\right)\left(\eta_{1}-u\right)^{2} d u \\
=\frac{30}{\eta_{1}^{5}}\left[\frac{\eta_{1}{ }^{2} u^{4}}{4}-\frac{2 \eta_{1} u^{5}}{5}+\frac{u^{6}}{6}\right]_{0}^{\eta}=\frac{\eta_{1}}{2}
\end{gathered}
$$




$$
\begin{gathered}
E\left(M^{2}\right)=\int_{0}^{\eta_{1}} u \frac{30}{\eta_{1}^{5}}\left(u^{2}\right)\left(\eta_{1}-u\right)^{2} d u=\left.\frac{30}{\eta_{1}^{5}}\left[\frac{\eta_{1}^{2} u^{5}}{5}-\frac{2 \eta_{1} u^{6}}{6}+\frac{u^{7}}{7}\right]\right|_{0} ^{\eta}=\frac{2 \eta_{1}^{2}}{7}=\frac{\eta_{1}^{2}(n+3)}{4(n+2)} \\
\operatorname{Var}(M)=\frac{2 \eta_{1}^{2}}{7}-\frac{\eta_{1}^{2}}{4}=\frac{\eta_{1}^{2}}{28}=\frac{\eta_{1}^{2}}{4(n+2)} .
\end{gathered}
$$

For $n=2 m+1$, then $M=U_{(m+1)}$.

$$
f_{M}(u)=\frac{(2 m+1) !}{(m) !(m) !}\left[\frac{u}{\eta_{1}}\right]^{(m)}\left[1-\frac{u}{\eta_{1}}\right]^{(m)} \frac{1}{\eta_{1}}=\frac{(2 m+1) !}{(m) !(m) ! \eta^{2 m+1}}\left(u^{(m)}\right)\left(\eta_{1}-u\right)^{(m)}, 0<u<\eta_{1}
$$

Now, let's assume that the following hold true for any $\mathrm{n}=2 \mathrm{~m}+1$.

$$
\begin{gathered}
E(M)=\int_{0}^{\eta_{1}} u \frac{(2 m+1) !}{(m) !(m) ! \eta_{1}^{2 m+1}}(u)^{m}\left(\eta_{1}-u\right)^{m}=\frac{\eta_{1}}{2} \\
E\left(M^{2}\right)=\int_{0}^{\eta_{1}} u^{2} \frac{(2 m+1) !}{(m) !(m) ! \eta_{1}^{2 m+1}}(u)^{m}\left(\eta_{1}-u\right)^{m} d u=\frac{\eta_{1}^{2}(2 m+4)}{4(2 m+3)}
\end{gathered}
$$

We need to show that for any $\mathrm{m}$ and for $\mathrm{n}=2 \mathrm{~m}+3$ the statements of the Theorem 3.4.1 are true.

For $\mathrm{n}=(2 \mathrm{~m}+3) \quad M=U_{(m+2)}$.

$$
\begin{gathered}
f_{M}(u)=\frac{(2 m+3) !}{(m+1) !(m+1) !}\left[\frac{u}{\eta_{1}}\right]^{(m+1)}\left[1-\frac{u}{\eta_{1}}\right]^{(m+1)} \frac{1}{\eta_{1}}=\frac{(2 m+1) !}{(m+1) !(m+1) ! \eta_{1}{ }^{2 m+2}}\left(u^{(m+1)}\right)\left(\eta_{1}-u\right)^{(m+1)}, 0<u<\eta_{1} \\
E(M)=\int_{0}^{\eta_{1}} u \frac{(2 m+3) !}{(m+1) !(m+1) ! \eta^{2 m+3}}\left(u^{(m+1)}\right)\left(\eta_{1}-u\right)^{(m+1)} d u .
\end{gathered}
$$

If we let $(m+1)=k$ then in accordance with (3.4.2) and (3.4.3) we conclude the following.

$$
\begin{gathered}
E(M)=\int_{0}^{\eta_{1}} u \frac{(2 k+1) !}{(k) !(k) ! \eta_{1}{ }^{2 k+1}}\left(u^{k}\right)\left(\eta_{1}-u\right)^{k} d u=\frac{\eta_{1}}{2} . \\
E\left(M^{2}\right)=\int_{0}^{\eta_{1}} u^{2} \frac{(2 m+3) !}{(m+1) !(m+1) ! \eta_{1}{ }^{2 m+3}}\left(u^{(m+1)}\right)\left(\eta_{1}-u\right)^{(m+1)} d u=\int_{0}^{\eta_{1}} u^{2} \frac{(2 k+1) !}{(k) !(k) ! \eta_{1}{ }^{2 k+1}}\left(u^{k}\right)\left(\eta_{1}-u\right)^{k} d u \\
\left.E\left(M^{2}\right)=\frac{\eta_{1}{ }^{2}(2 k+4)}{4(2 k+3)}=\frac{\eta_{1}{ }^{2}(2 m+6)}{4(2 m+5)}=\frac{\eta_{1}{ }^{2}(n+3)}{4(n+2)}, \quad \text { For that } \mathrm{n}=(2 \mathrm{~m}+3)\right), \text { and } \\
\operatorname{Var}(M)=\frac{\eta_{1}{ }^{2}(2 m+6)}{4(2 m+5)}-\frac{\eta_{1}{ }^{2}}{4}=\frac{\eta_{1}{ }^{2}}{4(2 m+5)}=\frac{\eta_{1}{ }^{2}}{4(n+2)} .
\end{gathered}
$$

For the case of $\mathrm{n}$ being an odd number, the proof is completed.

3.4.2. Estimation of the Parameter $\theta$ of the Distribution $X \sim \operatorname{Uniform}\left(\theta_{1}, b\right)$ by the Sample Median: $\mathrm{n}$ is even

Theorem 3.4.2 If $W \sim \operatorname{Uniform}\left(0, \eta_{1}\right)$ and if an even sized random sample is taken from this distribution then for the sample median M,

\section{Proof:}

$$
E(M)=\frac{\eta_{1}}{2}, E\left(M^{2}\right)=\frac{\left(n^{2}+4 n+2\right) \eta_{1}^{2}}{4(n+1)(n+2)}, \text { and } \operatorname{Var}(M)=\frac{n \eta_{1}^{2}}{4(n+1)(n+2)} .
$$

\section{Note:}

If $\mathrm{n}$ is even, then the sample median is $M=\frac{U_{n / 2}+U_{(n+2) / 2}}{2}$. 
To compute the expected value and the variance of M we need to have the joint pdf of $U_{n / 2}, U_{(n+2) / 2}$.

For any random sample taken from the distribution of $f(y)$, joint pdf of the ordered statistics $U_{r}$, and $U_{t},(\mathrm{r}<\mathrm{t})$ can be obtained by the use of the following general formulation as given in[6].

$$
\begin{gathered}
f_{U_{r}, U_{t}}\left(u_{r}, u_{t}\right)=\frac{n !}{(r-1) !(t-r-1) !(n-t) !}\left[F ( u _ { r } ] ^ { r - 1 } \left[F\left(u_{t}\right)-F\left(u_{r}\right]^{t-r-1}\left[F\left(u_{t}\right]^{n-t} f\left(u_{r}\right) f\left(u_{t}\right)\right.\right.\right. \\
\text { For } n=2: M=\frac{U_{1}+U_{2}}{2}=\frac{Y_{1}+Y_{2}}{2}=\bar{Y}
\end{gathered}
$$

If $W \sim \operatorname{Uniform}\left(0, \eta_{1}\right)$ then, $E(Y)=E(\bar{Y})=E(M)=\frac{\eta_{1}}{2}$, and

$$
\operatorname{Var}(\bar{Y})=\operatorname{Var}(M)=\frac{\operatorname{Var}(Y)}{n}=\frac{\eta_{1}^{2}}{12 n}=\frac{\eta_{1}^{2}}{24}=\frac{n \eta_{1}^{2}}{4(n+1)(n+2)} .
$$

For $n=4: M=\frac{U_{2}+U_{3}}{2}$. The joint $\operatorname{pdf}$ of $U_{2}$ and $U_{3}$ is obtained as given below.

Since, $f(u)=\frac{1}{\eta_{1}}$, and $F(u)=\int_{0}^{u} \frac{1}{\eta_{1}} d y=\frac{u}{\eta_{1}}, 0<u<\eta_{1}$ according to (3.4.5) the joint pdf is

$$
\begin{aligned}
& f_{U_{2}, U_{3}}\left(u_{2}, u_{3}\right)=\frac{4 !}{(1) !(0) !(1) !}\left[\frac{u_{2}}{\eta_{1}}\right]^{1}\left[1-\frac{u_{3}}{\eta_{1}}\right]^{1} \frac{1}{\eta_{1}{ }^{2}}=\frac{24}{\eta_{1}^{4}}\left(u_{2}\right)\left(\eta_{1}-u_{3}\right), 0<u_{2}<u_{3}<\eta_{1} \\
& E(M)=E\left(\frac{U_{2}+U_{3}}{2}\right)=\int_{0}^{\eta_{1}} \int_{0}^{u_{3}} \frac{u_{2}+u_{3}}{2}\left(\frac{24}{\eta_{1}^{4}}\right)\left(u_{2}\right)\left(\eta_{1}-u_{3}\right) d u_{2} d u_{3}= \\
& \frac{12}{\eta^{4}} \int_{0}^{\eta_{1}}\left(\frac{\eta_{1} u_{2}^{3}}{3}+\frac{\eta_{1} u_{3} u_{2}^{2}}{2}-\frac{u_{3} u_{2}^{3}}{3}-\frac{u_{3}^{2} u_{2}^{2}}{2}{\underset{u}{u_{2}=0}}_{u_{3}}^{u_{1}}\right) d u_{3}=\frac{12}{\eta_{1}^{4}} \int_{0}^{\eta_{1}}\left(\frac{\eta_{1} u_{3}^{3}}{3}+\frac{\eta_{1} u_{3}^{3}}{2}-\frac{u_{3}^{4}}{3}-\frac{u_{3}^{4}}{2}\right) d u_{3}= \\
& \left.\frac{12}{\eta_{1}^{4}}\left(\frac{\eta_{1} u_{3}^{4}}{12}+\frac{\eta_{1} u_{3}^{4}}{8}-\frac{u_{3}^{5}}{15}-\frac{u_{3}^{5}}{10}\right)\right|_{u_{3}=0} ^{\eta_{1}}=12 \eta_{1}\left(\frac{1}{12}+\frac{1}{8}-\frac{1}{15}-\frac{1}{10}\right)=\frac{\eta_{1}}{2} \\
& E\left(M^{2}\right)=E\left[\left(\frac{U_{2}+U_{3}}{2}\right)^{2}\right]=\int_{0}^{\eta_{1}} \int_{0}^{u_{3}}\left(\frac{u_{2}+u_{3}}{2}\right)^{2}\left(\frac{24}{\eta_{1}^{4}}\right)\left(u_{2}\right)\left(\eta_{1}-u_{3}\right) d u_{2} d u_{3}= \\
& \frac{6}{\eta_{1}^{4}} \int_{0}^{\eta_{1}}\left(\frac{\eta_{1} u_{2}^{4}}{4}+\frac{2 \eta_{1} u_{3} u_{2}^{3}}{3}+\frac{\eta_{1} u_{3}^{2} u_{2}^{2}}{2}-\frac{u_{3} u_{2}^{4}}{4}-\frac{2 u_{3}^{2} u_{2}^{3}}{3}-\frac{u_{3}^{3} u_{2}^{2}}{2}{\underset{u}{u_{2}=0}}_{\mid}^{u_{3}}\right) d u_{3} \\
& =\frac{6}{\eta_{1}^{4}} \int_{0}^{\eta_{1}}\left(\eta_{1} u_{3}^{4}\left(\frac{1}{4}+\frac{2}{3}+\frac{1}{2}\right)-u_{3}^{5}\left(\frac{1}{4}+\frac{2}{3}+\frac{1}{2}\right)\right) d u_{3}=\frac{6}{\eta_{1}^{4}}\left(\frac{17}{12}\right) \int_{0}^{\eta_{11}}\left(\eta_{1} u_{3}^{4}-u_{3}^{5}\right) d u_{3} \\
& \frac{17}{2 \eta_{1}^{4}}\left(\frac{\eta_{1} u_{3}^{5}}{5}-\frac{u_{3}^{6}}{6}\right) \underset{u_{3}=0}{\eta_{1}}=\frac{17 \eta_{1}^{2}}{2}\left(\frac{1}{5}-\frac{1}{6}\right)=\frac{17 \eta_{1}^{2}}{60} \\
& E\left(M^{2}\right)=\frac{17 \eta^{2}}{60} \rightarrow \quad E\left(M^{2}\right)=\frac{\left(n^{2}+4 n+2\right) \eta^{2}}{4(n+1)(n+2)}, \text { and } \\
& \operatorname{Var}(M)=\frac{17 \eta_{1}^{2}}{60}-\frac{\eta_{1}^{2}}{4}=\frac{\eta^{2}}{30} \rightarrow \operatorname{Var}(M)=\frac{n \eta_{1}^{2}}{4(n+1)(n+2)} .
\end{aligned}
$$

For $n=6: M=\frac{U_{3}+U_{4}}{2}$, the joint pdf of $U_{3}$ and $U_{4}$ is obtained as given below.: 


$$
\begin{aligned}
& f_{U_{3}, U_{4}}\left(u_{2}, u_{3}\right)=\frac{6 !}{(2) !(0) !(2) !}\left[\frac{u_{3}}{\eta_{1}}\right]^{2}\left[1-\frac{u_{4}}{\eta_{1}}\right]^{2} \frac{1}{\eta_{1}^{2}} \\
& =\frac{180}{\eta_{1}{ }^{6}}\left(u_{3}^{2}\right)\left(\eta_{1}-u_{4}\right)^{2}, 0<u_{3}<u_{4}<\eta_{1} \\
& E(M)=E\left(\frac{U_{3}+U_{4}}{2}\right)=\int_{0}^{\eta_{1} u_{4}} \frac{u_{3}+u_{4}}{2}\left(\frac{180}{\eta_{1}{ }^{6}}\right)\left(u_{3}^{2}\right)\left(\eta_{1}-u_{4}\right)^{2} d u_{2} d u_{3}= \\
& \frac{90}{\eta_{1}{ }^{6}} \int_{0}^{\eta_{1}}\left(\frac{\eta_{1}^{2} u_{3}^{4}}{4}+\frac{\eta_{1}^{2} u_{4} u_{3}^{3}}{3}-\frac{2 \eta_{1} u_{4} u_{3}^{4}}{4}-\frac{2 \eta_{1} u_{4}^{2} u_{3}^{3}}{3}+\frac{u_{4}^{2} u_{3}^{4}}{4}+\frac{u_{4}^{3} u_{3}^{3}}{3}\right) d u_{3}=\rightarrow \text { for } n=6, E(M)=\frac{\eta_{1}}{2} \\
& \frac{90}{\eta^{6}}\left(\frac{7}{12}\right) \int_{0}^{\eta_{1}}\left(\eta_{1}^{2} u_{4}^{4}-2 \eta u_{4}^{5}+u_{4}^{6}\right) d u_{3}=\left.\frac{105}{2 \eta_{1}^{6}}\left(\frac{\eta_{1}^{2} u_{4}^{5}}{5}-\frac{2 \eta_{1} u_{4}^{6}}{6}+\frac{u_{4}^{7}}{7}\right)\right|_{0} ^{\eta_{1}} \\
& \frac{105 \eta_{1}}{2}\left(\frac{1}{5}-\frac{1}{3}+\frac{1}{7}\right)=\frac{\eta_{1}}{2} \\
& E\left(M^{2}\right)=E\left[\left(\frac{U_{3}+U_{4}}{2}\right)^{2}\right]=\int_{0}^{\eta_{1}} \int_{0}^{u_{4}}\left(\frac{u_{3}+u_{4}}{2}\right)^{2}\left(\frac{180}{\eta_{1}{ }^{6}}\right)\left(u_{3}^{2}\right)\left(\eta_{1}-u_{4}\right)^{2} d u_{2} d u_{3}= \\
& \frac{45}{\eta^{6}} \int_{0}^{\eta_{1}}\left(\frac{\eta_{1}^{2} u_{3}^{5}}{5}+\frac{2 \eta_{1}^{2} u_{4} u_{3}^{4}}{4}+\frac{\eta_{1}^{2} u_{4}^{2} u_{3}^{3}}{3}-\frac{2 \eta_{1} u_{4} u_{3}^{5}}{5}-\frac{4 \eta_{1} u_{4}^{2} u_{3}^{4}}{4}-\frac{2 \eta_{1} u_{4}^{3} u_{3}^{3}}{3}+\frac{u_{4}^{2} u_{3}^{5}}{5}+\frac{2 u_{4}^{2} u_{3}^{4}}{4}+\left.\frac{u_{4}^{4} u_{3}^{3}}{3}\right|_{u_{3}=0} ^{u_{4}}\right) d u_{4}= \\
& \frac{45}{\eta_{1}{ }^{6}}\left(\frac{31}{30}\right)_{0}^{\eta_{1}}\left(\eta_{1}^{2} u_{4}^{5}-2 \eta_{1} u_{4}^{6}+u_{4}^{7}\right) d u_{4}=\frac{93}{2 \eta_{1}^{6}}\left(\frac{\eta_{1}^{2} u_{4}^{6}}{6}-\frac{2 \eta_{1} u_{4}^{7}}{7}+\frac{u_{4}^{8}}{8}\right)_{0}^{\eta_{1}} \\
& \frac{93 \eta_{1}^{2}}{2}\left(\frac{1}{6}-\frac{2}{7}+\frac{1}{8}\right)=\frac{31 \eta_{1}^{2}}{112} \\
& E\left(M^{2}\right)=\frac{31 \eta_{1}^{2}}{112} \rightarrow \quad E\left(M^{2}\right)=\frac{\left(n^{2}+4 n+2\right) \eta_{1}{ }^{2}}{4(n+1)(n+2)}, \text { and } \\
& \operatorname{Var}(M)=\frac{31 \eta_{1}^{2}}{112}-\frac{\eta_{1}^{2}}{4}=\frac{3 \eta_{1}^{2}}{112} \rightarrow \operatorname{Var}(M)=\frac{n \eta_{1}^{2}}{4(n+1)(n+2)} \\
& \text { For } n=2 m+2: \quad M=\frac{U_{(m+1)}+U_{(m+2)}}{2} \text {. } \\
& f_{U_{(m+1)}, U_{(m+2)}}\left(u_{m+1}, u_{m+2}\right)=\frac{(2 m+2) !}{(m) !(0) !(m) !}\left[\frac{u_{m+1}}{\eta_{1}}\right]^{m}\left[1-\frac{u_{m+2}}{\eta_{1}}\right]^{m} \frac{1}{\eta_{1}^{2}} \\
& =\frac{(2 m+2) !}{m ! m ! \eta_{1}^{2 m+2}}\left(u_{m+1}^{m}\right)\left(\eta_{1}-u_{m+2}\right)^{m}, 0<u_{m+1}<u_{m+2}<\eta_{1}
\end{aligned}
$$

Now, let's assume that the following, given in (3.4.7) and (3.48), hold true.

$$
E(M)=\int_{0}^{\eta_{1} u_{m+2}} \frac{u_{m+1}+u_{m+2}}{2} \frac{(2 m+2) !}{m ! m ! \eta_{1}^{2 m+2}}\left(u_{m+1}^{m}\right)\left(\eta_{1}-u_{m+2}\right)^{m} d u_{m+1} d u_{m+2}=\frac{\eta_{1}}{2}
$$




$$
\begin{aligned}
& E\left(M^{2}\right)=\int_{0}^{\eta_{1}} \int_{0}^{u_{m+2}}\left(\frac{u_{m+1}+u_{m+2}}{2}\right)^{2} \frac{(2 m+2) !}{m ! m ! \eta_{1}^{2 m+2}}\left(u_{m+1}^{m}\right)\left(\eta_{1}-u_{m+2}\right)^{m} d u_{m+1} d u_{m+2}= \\
& \frac{\left[(2 m+2)^{2}+4(2 m+2)+2\right] \eta_{1}^{2}}{4[(2 m+2)+1][(2 m+2)+2]}=\frac{\left[n^{2}+4(n+2)+2\right] \eta_{1}^{2}}{4(n+1)(n+2)}
\end{aligned}
$$

We need to show that the statements of the Theorem 3.4.2 hold true for $n=2 m+4$.

For $\mathrm{n}=2 \mathrm{~m}+4, \quad M=\frac{U_{(m+2)}+U_{(m+3)}}{2}$

The joint pdf of $U_{(m+2)}$ and $U_{(m+3)}$ is obtained as follows.

$$
\begin{aligned}
& f_{U_{(m+2)}, U_{(m+3)}}\left(u_{m+2}, u_{m+3}\right)=\frac{(2 m+4) !}{(m+1) !(0) !(m+) !}\left[\frac{u_{m+1}}{\eta_{1}}\right]^{m+1}\left[1-\frac{u_{m+2}}{\eta_{1}}\right]^{m+1} \frac{1}{\eta_{1}^{2}} \\
& =\frac{(2 m+4) !}{(m+1) !(m+1) ! \eta_{1}^{2 m+4}}\left(u_{m+2}^{m+1}\right)\left(\eta_{1}-u_{m+3}\right)^{m+1}, 0<u_{m+2}<u_{m+3}<\eta_{1} \\
& E(M)=\int_{0}^{\eta_{1} \int_{m+3}^{u_{m+3}}} \frac{u_{m+2}+u_{m+3}}{2} \frac{(2 m+4) !}{(m+1) !(m+1) ! \eta_{1}{ }^{2 m+4}}\left(u_{m+1}^{m+1}\right)\left(\eta_{1}-u_{m+2}\right)^{m+1} d u_{m+2} d u_{m+3}
\end{aligned}
$$

In (3.4.9 take $(m+1)=k$, observing the identity between (3.4.7) and (3.4.10) we conclude the following.

$$
E(M)=\int_{0}^{\eta_{1}} \int_{0}^{u_{k+2}} \frac{u_{k+1}+u_{k+2}}{2} \frac{(2 k+2) !}{k ! k ! \eta_{1}^{2 k+2}}\left(u_{k+1}^{k}\right)\left(\eta_{1}-u_{k+2}\right)^{k} d u_{k+1} d u_{k+2}=\frac{\eta_{1}}{2}
$$

Similarly,

$$
E\left(M^{2}\right)=\int_{0}^{\eta_{1}} \int_{0}^{u_{m+3}}\left(\frac{u_{m+2}+u_{m+3}}{2}\right)^{2} \frac{(2 m+4) !}{(m+1) !(m+1) ! \eta_{1}^{2 m+4}}\left(u_{m+1}^{m+1}\right)\left(\eta_{1}-u_{m+2}\right)^{m+1} d u_{m+2} d u_{m+3}
$$

In (3.4.11), if we let $(m+1)=k$, observing the identity between (3.4.8) and (3.4.12) we conclude the following.

$$
\begin{aligned}
& E\left(M^{2}\right)=\int_{0}^{\eta_{1}} \int_{0}^{u_{k+2}}\left(\frac{u_{k+1}+u_{k+2}}{2}\right)^{2} \frac{(2 k+2) !}{k ! k ! \eta_{1}^{2 k+2}}\left(u_{k+1}^{k}\right)\left(\eta_{1}-u_{k+2}\right)^{k} d u_{k+1} d u_{k+2}= \\
& \frac{\left[(2 k+2)^{2}+4(2 k+2)+2\right] \eta_{1}^{2}}{4[(2 k+2)+1][(2 k+2)+2]}=\frac{\left[(2 m+4)^{2}+4(2 m+4)+2\right] \eta_{1}^{2}}{4[(2 m+4)+1][(2 m+4)+2]}=\frac{\left(n^{2}+4 n+2\right) \eta_{1}^{2}}{4(n+1)(n+2)}
\end{aligned}
$$

For the case of $\mathrm{n}$ being an even number, the proof is completed.

3.4.3. Unbiased Estimators of $\theta_{1}$ for $X \sim \operatorname{Uniform}\left(\theta_{1}, b\right)$ by $\mathrm{M}$

$n$ is odd: $E(M)=\frac{\eta_{1}}{2}, E\left(M^{2}\right)=\frac{\eta_{1}^{2}(n+3)}{4(n+2)}$, and $\operatorname{Var}(M)=\frac{\eta_{1}{ }^{2}}{4(n+2)}$

Where $\eta_{1}=b-\theta_{1}$, hence an unbiased estimator of $\theta_{1}$ as function of is $\mathrm{M}$

$$
T_{4}=b-2 M, \operatorname{Var}\left(T_{4}\right)=4 \operatorname{Var}(M)=\frac{\left(b-\theta_{1}\right)^{2}}{(n+2)}
$$

$n$ is even: $E(M)=\frac{\eta_{1}}{2}, E\left(M^{2}\right)=\frac{\left(n^{2}+4 n+2\right) \eta_{1}{ }^{2}}{4(n+1)(n+2)}$, and $\operatorname{Var}(M)=\frac{n \eta_{1}{ }^{2}}{4(n+1)(n+2)}$. 
Table 1. Unbiased estimators of $\theta_{1}$ for the Distribution $X \sim \operatorname{Uniform}\left(\theta_{1}, b\right)$ and their variances

\begin{tabular}{|c|c|c|c|c|c|}
\hline & \multicolumn{5}{|c|}{ Unbiased estimators $\left(T_{i}\right)$} \\
\hline & $T_{1}=\frac{(n+1) Y_{1}-b}{n}$ & $T_{2}=(n+1) Y_{n}-n b$ & $T_{3}=2(\bar{X})-b$ & $T_{4}=b-2 M$ & $T_{5}=b-2 M$ \\
(n is odd) & (n is even) \\
\hline$E\left(T_{i}\right)$ & $\theta_{1}$ & $\theta_{1}$ & $\theta_{1}$ & $\theta_{1}$ & $\theta_{1}$ \\
\hline $\operatorname{Var}\left(T_{i}\right)$ & $\frac{\left(b-\theta_{1}\right)^{2}}{n(n+2)}$ & $\frac{n\left(b-\theta_{1}\right)^{2}}{(n+2)}$ & $\frac{\left(b-\theta_{1}\right)^{2}}{3 n}$ & $\frac{\left(b-\theta_{1}\right)^{2}}{(n+2)}$ & $\frac{n\left(b-\theta_{1}\right)^{2}}{(n+1)(n+2)}$ \\
\hline
\end{tabular}

Where $\eta_{1}=b-\theta_{1}$, hence an unbiased estimator of $\theta$ as function of is $\mathrm{M} T_{5}=a-2 M$,

$$
\operatorname{Var}\left(T_{5}\right)=4 \operatorname{Var}(M)=\frac{n\left(b-\theta_{1}\right)^{2}}{(n+1)(n+2)}
$$

If we give the above comparisons in a tabulated form, we obtain the following:

For

$\mathrm{n}>1$;

$\operatorname{Var}\left(T_{1}\right)<\operatorname{Var}\left(T_{3}\right)<\operatorname{Var}\left(T_{5}\right)<\operatorname{Var}\left(T_{4}\right)<\operatorname{Var}\left(T_{2}\right)$

We see that, for $\mathrm{n}>1$, the most efficient unbiased estimator, among the ones as given above, is $T_{1}=\frac{(n+1) Y_{1}-b}{n}$.

\section{Confidence Interval for the Parameter}

\section{$\theta_{1}$ of the Distribution $X \sim \operatorname{Uniform}\left(\theta_{1}, b\right)$}

The most efficient unbiased estimator of $\theta_{1}$ is seen to be $T_{1}=\frac{(n+1) Y_{1}-b}{n}$. By the use of the pdf of $Y_{1}$ we can construct a $100(1-\alpha) \%$ confidence interval for $\theta_{1}$. As it is shown before

$$
f_{Y_{1}}(y)=\frac{n}{\left(b-\theta_{1}\right)^{n}}(b-y)^{(n-1)}, \theta_{1}<y<b .
$$

By the use of following probability statement we can obtain a confidence interval for $\theta$.

$$
\begin{gathered}
P\left(y_{1 L}<Y_{1}<y_{1 U}\right)=(1-\alpha) \\
\int_{\theta_{1}}^{y_{1 L}} \frac{n}{(b-\theta)^{n}}(b-y)^{(n-1)} d y=\alpha / 2 \text { and }
\end{gathered}
$$

$$
\int_{\theta_{1}}^{y_{1 U}} \frac{n}{\left(b-\theta_{1}\right)^{n}}(b-y)^{(n-1)} d y=1-\alpha / 2
$$

If we let $(b-y)=w$ then the following are true:

$$
d w=-d y ; \quad y=\theta_{1} \rightarrow w=b-\theta_{1} ;
$$

$y=y_{1 L} \rightarrow w=b-y_{1 L}$, and $y=y_{1 U} \rightarrow w=b-y_{1 U}$.

$$
\begin{aligned}
& \int_{\theta_{1}}^{y_{1 L}} \frac{n}{\left(b-\theta_{1}\right)^{n}}(b-y)^{(n-1)} d y= \\
& \int_{b-y_{1 L}}^{b-\theta_{1}} \frac{n}{(b-\theta)^{n}} w^{n-1} d w=1-\left(\frac{b-y_{1 L}}{b-\theta_{1}}\right)^{n}=\alpha / 2
\end{aligned}
$$

$$
y_{1 L}=b-\left(b-\theta_{1}\right)\left(1-\frac{\alpha}{2}\right)^{1 / n}
$$

Similarly,

$$
\begin{gathered}
\int_{\theta_{1}}^{y_{1 U}} \frac{n}{\left(b-\theta_{1}\right)^{n}}(b-y)^{(n-1)} d y= \\
\int_{b-y_{1 U}}^{b-\theta_{1}} \frac{n}{\left(b-\theta_{1}\right)^{n}} w^{n-1} d w=1-\left(\frac{b-y_{1 U}}{b-\theta_{1}}\right)^{n}=1-\alpha / 2 \\
y_{1 U}=b-\left(b-\theta_{1}\right)\left(\frac{\alpha}{2}\right)^{1 / n}
\end{gathered}
$$

If the results in (4.2) and (4.3) are substituted in (4.1) 


$$
P\left(b-\left(b-\theta_{1}\right)\left(1-\frac{\alpha}{2}\right)^{1 / n}<Y_{1}<b-\left(b-\theta_{1}\right)\left(\frac{\alpha}{2}\right)^{1 / n}\right)=(1-\alpha)
$$

Solving the above inequalities for $\theta_{1}$ we obtain the following Confidence Interval.

$$
P\left(b-\frac{b-Y_{1}}{\left(\frac{\alpha}{2}\right)^{1 / n}}<\theta_{1}<b-\frac{b-Y_{1}}{\left(1-\frac{\alpha}{2}\right)^{1 / n}}\right)=(1-\alpha)
$$

A $(1-\alpha) * 100 \%$ confidence interval for $\theta_{1}$ :

$$
\left(b-\frac{b-Y_{1}}{\left(\frac{\alpha}{2}\right)^{1 / n}}, b-\frac{b-Y_{1}}{\left(1-\frac{\alpha}{2}\right)^{1 / n}}\right)
$$

\section{Tests of Hypotheses Related to the Parameter $\theta_{1}$ of the Distribution}

$$
X \sim \operatorname{Uniform}\left(\theta_{1}, b\right)
$$

Table 2. Tests of Hypotheses Related to the Parameter $\theta_{1}$ for the Distribution $X \sim \operatorname{Uniform}\left(\theta_{1}, b\right)$

\begin{tabular}{|c|c|c|}
\hline$H_{0}: \theta_{1}=\theta_{0}$ & $H_{0}: \theta_{1} \leq \theta_{0}$ & $H_{0}: \theta_{1} \geq \theta_{0}$ \\
$H_{1}: \theta_{1} \neq \theta_{0}$ & $H_{1}: \theta_{1}>\theta_{0}$ & $H_{1}: \theta_{1}<\theta_{0}$ \\
\hline If $Y_{1} \geq y_{1 U}$ or $Y_{1} \leq y_{1 L}$ & If $Y_{1} \geq y_{1 U} H_{0}$ is rejected & If $Y_{1} \leq y_{1 L} H_{0}$ is rejected \\
$H_{0}$ is rejected & Don't reject $\mathrm{H}_{0}$ otherwise & Don't reject $\mathrm{H}_{0}$ otherwise \\
\hline Don't reject $\mathrm{H}_{0}$ otherwise & & \\
\hline Where, & Where, \\
$y_{1 L}=b-\left(b-\theta_{0}\right)\left(1-\frac{\alpha}{2}\right)^{1 / n}$ & $y_{1 U}=b-\left(b-\theta_{0}\right)(\alpha)^{1 / n}$ & $y_{1 L}=b-\left(b-\theta_{0}\right)(1-\alpha)^{1 / n}$ \\
and & & Where \\
$y_{1 U}=b-\left(b-\theta_{0}\right)\left(\frac{\alpha}{2}\right)^{1 / n}$ & & \\
\hline
\end{tabular}


If $H_{0}: \theta_{1}=\theta_{0}$ is to be tested against to any proper alternative hypothesis, a plausible test statistic is to be $Y_{1}=X_{\text {Min }}$ for that the most efficient unbiased estimator is $T_{1}=\frac{(n+1) Y_{1}-b}{n}$, which is a linear function of $Y_{1}=X_{\text {Min }}$.

If the level of significance is chosen to be $\alpha$, then the decision rules, as given in the following table, are applicable.

It is concluded that the best unbiased estimator, among the ones suggested, of the parameter $\theta_{1}$ for the uniform distribution over $\left(\theta_{1}, b\right)$ is $T_{1}=\frac{(n+1) Y_{1}-b}{n}$.

Since $T_{1}$ is a linear function of the first order statistic $Y_{1}$, construction of confidence interval and tests of hypotheses procedures are related to and dependent upon the observed value of the first order statistic $Y_{1}$ and the chosen level of significance $\alpha$.

\section{Statistical Inferences Related to the Parameter of $X \sim \operatorname{Uniform}\left(a, \theta_{2}\right)$ Distribution}

\subsection{Estimation of the Parameter $\theta_{2}$ of the Distribution $X \sim \operatorname{Uniform}\left(a, \theta_{2}\right)$ by the First Order Statistic}

$$
Y_{1}=X_{(\text {Min })}
$$

A uniformly distributed continuous random variable $\mathrm{X}$, over the interval $\left(a, \theta_{2}\right)$, has the following pdf.

$$
f(x)=\frac{1}{\theta_{2}-a}, a<x<\theta_{2}
$$

If $Y=X-a$ then, $Y \sim \operatorname{Uniform}\left(0,\left(\theta_{2}-a\right)=\eta\right)$ and its pdf is as given below.

$$
f(y)=\frac{1}{\eta}, 0<y<\eta \text {. }
$$

If a random sample of size is taken from the distribution of $\mathrm{Y}$, then the ordered statistics will be denoted by $U_{1}<U_{2}<\ldots<U_{n}$.

The parameter of this distribution, $\eta$, can be estimated by $U_{1}$. The pdf of $U_{1}$ is given below.

$$
\begin{gathered}
f_{U_{1}}(y)=n\left[1-\frac{y}{\eta}\right]^{(n-1)} \frac{1}{\eta}=\frac{n}{(\eta)^{n}}(\eta-y)^{(n-1)}, 0<y<\eta \\
E\left(U_{1}\right)=\frac{n}{(\eta)^{n}} \int_{\theta}^{a} y(\eta-y)^{(n-1)} d y
\end{gathered}
$$

If we let $(\eta-y)=t$ then the following will hold true.

$$
\begin{aligned}
& d t=-d y ; \quad y=\eta-t ; \quad y=\theta \rightarrow t=\eta-\theta_{2} \quad y=a \rightarrow t=0 . \\
& E\left(U_{1}\right)=\frac{n}{(\eta)^{n}} \int_{0}^{\eta} y(\eta-y)^{(n-1)} d y=\frac{n}{(\eta)^{n}} \int_{0}^{\eta}(\eta-t) t^{(n-1)} d t= \\
& \left.\frac{n}{(\eta)^{n}}\left[\frac{\eta t^{n}}{n}-\frac{t^{n+1}}{n+1}\right]\right|_{t=0} ^{(\eta)}=n \eta\left[\frac{1}{n}-\frac{1}{n+1}\right] \\
& E\left(U_{1}\right)=\frac{\eta}{n+1}
\end{aligned}
$$

Hence, an estimator of $\theta_{2}$ of $X \sim \operatorname{Uniform}\left(a, \theta_{2}\right)$ will be $X_{(1)}$ and its expected value, from the transformation $Y=X-a$, is obtained as given below. 


$$
E\left(Y_{1}\right)=E\left(U_{1}\right)+a=\frac{\eta}{(n+1)}+a=\frac{\left(\theta_{2}-a\right)}{(n+1)}+a=\frac{n a+\theta_{2}}{(n+1)}
$$

Similarly,

$$
\begin{aligned}
& E\left(U_{1}^{2}\right)=\frac{n}{(\eta)^{n}} \int_{0}^{\eta} y^{2}(\eta-y)^{(n-1)}=\frac{n}{(\eta)^{n}} \int_{0}^{\eta}(\eta-t)^{2} t^{(n-1)} d t= \\
& \left.\frac{n}{(\eta)^{n}}\left[\frac{\eta^{2} t^{n}}{n}-\frac{2 \eta t^{n+1}}{n+1}+\frac{t^{n+2}}{n+2}\right]\right|_{t=0} ^{(\eta)}=n \eta^{2}\left[\frac{1}{n}-\frac{2}{n+1}+\frac{1}{n+2}\right] \rightarrow E\left(U_{1}^{2}\right)=\frac{2 \eta^{2}}{(n+1)(n+2)}
\end{aligned}
$$

By using (6.1.3) and (6.1.5) we obtained the variance of $U_{1}$ and $Y_{1}$.

$$
\begin{aligned}
& \operatorname{Var}\left(U_{1}\right)=E\left(U_{1}^{2}\right)-\left[E\left(U_{1}\right)\right]^{2}=\frac{2 \eta^{2}}{(n+1)(n+2)}-\frac{(\eta)^{2}}{(n+1)^{2}}= \\
& \operatorname{Var}\left(Y_{1}\right)=\frac{n(\eta)^{2}}{(n+1)^{2}(n+2)}
\end{aligned}
$$

Since, $Y=X-a$, then $\operatorname{Var}\left(Y_{1}\right)=\operatorname{Var}(Y)=\operatorname{Var}\left(U_{1}\right)$.

$$
\operatorname{Var}\left(Y_{1}\right)=\frac{n(\eta)^{2}}{(n+1)^{2}(n+2)}=\frac{n\left(\theta_{2}-a\right)^{2}}{(n+1)^{2}(n+2)}
$$

By the utilization of (6.1.3) we can obtain an unbiased estimator for $\theta$ as a function of $Y_{1}$.

$$
W_{1}=(n+1) Y_{1}-n a, \text { and } \operatorname{Var}\left(W_{1}\right)=(n+1)^{2} \operatorname{Var}\left(Y_{1}\right)=\frac{n\left(\theta_{2}-a\right)^{2}}{n(n+2)}
$$

6.2. Estimation of the Parameter $\theta$ of the Distribution $X \sim \operatorname{Uniform}\left(a, \theta_{2}\right)$ by the Last Order Statistic

$$
Y_{n}=X_{(\operatorname{Max})}
$$

If we want to estimate the parameter $\eta$ of $Y \sim \operatorname{Uniform}(0, \eta)$ by $U_{n}$,

$$
\begin{gathered}
f_{U_{n}}(y)=n\left[\frac{y}{\eta}\right]^{(n-1)} \frac{1}{\eta}=\frac{n}{(\eta)^{n}}(y)^{(n-1)}, 0<y<\eta \\
E\left(U_{n}\right)=\frac{n}{(\eta)^{n}} \int_{\theta}^{a} y(y)^{(n-1)} d y \\
E\left(U_{n}\right)=\frac{n}{(\eta)^{n}} \int_{0}^{\eta} y(y)^{(n-1)} d y=\left.\frac{n}{(\eta)^{n}}\left[\frac{y^{n+1}}{n+1}\right]\right|_{t=0} ^{(\eta)}=\left[\frac{n \eta}{n+1}\right]
\end{gathered}
$$

The Maximum Likelihood estimator for the parameter $\theta_{2}$ is $Y_{n}$.

Since, $Y=X-a$, then the following will be true.

$$
\begin{aligned}
& E\left(Y_{n}\right)=E\left(U_{n}\right)+a=\frac{n \eta}{(n+1)}+a=\frac{n(\theta-a)}{(n+1)}+a=\frac{n \theta_{2}+a}{(n+1)} \\
& E\left(U_{n}^{2}\right)=\frac{n}{(\eta)^{n}} \int_{0}^{\eta} y^{2}(y)^{(n-1)} d y=\left.\frac{n}{(\eta)^{n}}\left[\frac{y^{n+2}}{n+2}\right]\right|_{t=0} ^{(\eta)}=\left[\frac{n \eta^{2}}{n+2}\right]
\end{aligned}
$$




$$
\begin{gathered}
\operatorname{Var}\left(U_{n}\right)=E\left(U_{n}^{2}\right)-\left[E\left(U_{n}\right)\right]^{2}=\frac{n \eta^{2}}{(n+2)}-\frac{(n \eta)^{2}}{(n+1)^{2}}= \\
\operatorname{Var}\left(U_{n}\right)=\frac{n(\eta)^{2}}{(n+1)^{2}(n+2)}=\operatorname{Var}\left(Y_{n}\right) \\
\operatorname{Var}\left(Y_{n}\right)=\operatorname{Var}(Y)=\operatorname{Var}\left(U_{n}\right) .
\end{gathered}
$$

From (6.2.4) we obtain an unbiased estimator for $\theta$ as a function of $Y_{n}$.

$$
W_{2}=\frac{(n+1) Y_{n}-a}{n}, \text { and } \operatorname{Var}\left(W_{2}\right)=\frac{(n+1)^{2} \operatorname{Var}\left(X_{(n)}\right.}{n^{2}}=\frac{\left(\theta_{2}-a\right)^{2}}{n(n+2)}
$$

6.3. Estimation of the Parameter $\theta_{2}$ of the Distribution $X \sim \operatorname{Uniform}\left(a, \theta_{2}\right)$ by the Sample Mean $\bar{X}$

$$
\begin{aligned}
& \text { If } X \sim \text { Uniform }(a, \theta) \text { then the pdf is } \\
& f(x)=\frac{1}{\theta_{2}-a}, a<x<\theta_{2}, \\
& E(X)=\int_{a}^{\theta} x \frac{1}{\theta_{2}-a} d x=\frac{\left(\theta_{2}^{2}-a^{2}\right)}{2\left(\theta_{2}-a\right)}=\frac{\left(a+\theta_{2}\right)}{2}, \\
& E\left(X^{2}\right)=\int_{a}^{\theta} x^{2} \frac{1}{\theta_{2}-a} d x=\frac{\left(\theta_{2}^{3}-a^{3}\right)}{3\left(\theta_{2}-a\right)}=\frac{\left(a^{2}+a \theta_{2}+\theta_{2}^{2}\right)}{3}, \\
& \operatorname{Var}(X)=\frac{(\theta-a)^{2}}{12} .
\end{aligned}
$$

For any distribution, if the sample mean is $\bar{X}$, for any random sample of size $\mathrm{n}$, the following hold true.

$$
\begin{gathered}
E(\bar{X})=E(X), \text { and } \operatorname{Var}(\bar{X})=\frac{\operatorname{Var}(X)}{n} \\
E(\bar{X})=\frac{\left(a+\theta_{2}\right)}{2} \\
\text { And } \operatorname{Var}(\bar{X})=\frac{\left(\theta_{2}-a\right)^{2}}{12 n}
\end{gathered}
$$

From (4.3.1) we can obtain an unbiased estimator for $\theta$, as function of the sample mean $\bar{X}$.

$$
\begin{gathered}
W_{3}=2(\bar{X})-a, \text { and } \\
\operatorname{Var}\left(W_{3}\right)=4 \operatorname{Var}(\bar{X})=\frac{\left(a-\theta_{2}\right)^{2}}{3 n}
\end{gathered}
$$

\subsection{Estimation of the Parameter $\theta_{2}$ of the Distribution}

$$
X \sim \operatorname{Uniform}\left(a, \theta_{2}\right) \text { by the Median M }
$$

A uniformly distributed continuous random variable $\mathrm{X}$, over the interval $\left(a, \theta_{2}\right)$, has the following pdf

$$
f(x)=\frac{1}{\theta_{1}-a}, a<x<\theta_{2}
$$

If $Y=X-a$, then as it is stated in Note 1 , $Y \sim \operatorname{Uniform}\left(0,\left(\theta_{2}-a\right)=\eta\right)$ and its pdf is as given below.

$$
f(y)=\frac{1}{\eta}, 0<y<\eta .
$$

Estimation procedures and the findings, related to the parameter $\theta_{2}$ of $X \sim \operatorname{Uniform}\left(a, \theta_{2}\right)$, will be exactly the same as the one given in sections 1.4.1 and 1.4.2, with the exception that $\eta=\left(\theta_{2}-a\right)$

In other words, the statements of Theorem 1.4.1 and Theorem 1.4.2 hold true.

That is: if $\mathrm{n}$ is odd then, $E(M)=\frac{\eta}{2}$, $E\left(M^{2}\right)=\frac{\eta^{2}(n+3)}{4(n+2)}$, and $\operatorname{Var}(M)=\frac{\eta^{2}}{4(n+2)}$,

If $n$ is even, then

$$
\begin{gathered}
E(M)=\frac{\eta}{2}, E\left(M^{2}\right)=\frac{\left(n^{2}+4 n+2\right)}{4(n+1)(n+2)} \\
\operatorname{Var}(M)=\frac{n \eta^{2}}{4(n+1)(n+2)}, \text { where } \\
\eta=\left(\theta_{2}-a\right)
\end{gathered}
$$

The unbiased estimators of $\theta_{2}$ as functions of the sample median $\mathrm{M}$ are as follows:

$W_{4}=a+2 M$ (when $\mathrm{n}$ is odd), $W_{5}=a+2 M($ when $\mathrm{n}$ is even).

The variances of these unbiased estimators are:

$\operatorname{Var}\left(W_{4}\right)=\frac{\left(\theta_{2}-a\right)^{2}}{(n+2)}$, and $\operatorname{Var}\left(W_{5}\right)=\frac{n\left(\theta_{2}-a\right)^{2}}{(n+1)(n+2)}$

\subsection{Comparisons of Unbiased Estimators in Terms of} Their Efficiencies 
The unbiased estimators and their comparisons are given in Table 3.

We see that, for $\mathrm{n}>1$, the most efficient unbiased estimator among the ones given above, is $W_{2}=\frac{(n+1) Y_{n}-a}{n}$.

\section{Confidence Interval for the Parameter}

$\theta$ of the Distribution $X \sim \operatorname{Uniform}\left(a, \theta_{2}\right)$
The most efficient unbiased estimator of $\theta$ is seen to be $T_{2}=\frac{(n+1) Y_{n}-a}{n}$. By the use of the pdf of $Y_{n}$ we can construct a $100(1-\alpha) \%$ confidence interval for $\theta$. As it is shown before

$$
f_{Y_{n}}(y)=\frac{n}{\left(\theta_{2}-a\right)^{n}}(y-a)^{(n-1)}, a<y<\theta_{2} .
$$

By the use of following probability statement we can obtain a confidence interval for $\theta$.

$$
\begin{gathered}
P\left(y_{n L}<Y_{n}<y_{n U}\right)=(1-\alpha) \\
\int_{a}^{y_{n L}} \frac{n}{\left(\theta_{2}-a\right)^{n}}(y-a)^{(n-1)} d y=\alpha / 2 \text { And, } \int_{a}^{y_{n U}} \frac{n}{\left(\theta_{2}-a\right)^{n}}(y-a)^{(n-1)} d y=1-\alpha / 2
\end{gathered}
$$

If we let $(y-a)=w$ then the following are true:

$$
\begin{gathered}
d w=d y ; \quad y=a \rightarrow w=0 ; y=y_{n L} \rightarrow w=y_{n L}-a, \text { and } y=y_{n U} \rightarrow w=y_{n U}-a . \\
\int_{a}^{y_{n L}} \frac{n}{\left(\theta_{2}-a\right)^{n}}(y-a)^{(n-1)} d y=\int_{0}^{y_{n L-a}} \frac{n}{\left(\theta_{2}-a\right)^{n}} w^{n-1} d w=\left(\frac{y_{n L}-a}{\theta_{2}-a}\right)^{n}=\alpha / 2 \\
y_{n L}=a+\left(\theta_{2}-a\right)\left(\frac{\alpha}{2}\right)^{1 / n}
\end{gathered}
$$

Similarly,

$$
\begin{gathered}
\int_{a}^{y_{n U}} \frac{n}{\left(\theta_{2}-a\right)^{n}}(y-a)^{(n-1)} d y=\int_{0}^{y_{n U-a}} \frac{n}{\left(\theta_{2}-a\right)^{n}} w^{n-1} d w=\left(\frac{a-y_{n U}}{\theta_{2}-a}\right)^{n}=1-\alpha / 2 \\
y_{n U}=a+\left(\theta_{2}-a\right)\left(1-\frac{\alpha}{2}\right)^{1 / n}
\end{gathered}
$$

Table 3. Unbiased estimators of $\theta_{2}$ for the Distribution $X \sim \operatorname{Uniform}\left(a, \theta_{2}\right)$ and their variances

\begin{tabular}{|c|c|c|c|c|c|}
\hline & \multicolumn{5}{|c|}{ Unbiased estimators $\left(T_{i}\right)$} \\
\hline & $W_{1}=(n+1) Y_{1}-n a$ & $W_{2}=\frac{(n+1) Y_{n}-a}{n}$ & $W_{3}=2(\bar{X})-a$ & $W_{4}=a+2 M$ & $W_{5}=a+2 M$ \\
(n is odd) & (n is even) \\
\hline$E\left(W_{i}\right)$ & $\theta_{2}$ & $\theta_{2}$ & $\theta_{2}$ & $\theta_{2}$ & $\theta_{2}$ \\
\hline $\operatorname{Var}\left(W_{i}\right)$ & $\frac{n\left(\theta_{2}-a\right)^{2}}{(n+2)}$ & $\frac{\left(\theta_{2}-a\right)^{2}}{n(n+2)}$ & $\frac{\left(\theta_{2}-a\right)^{2}}{3 n}$ & $\frac{\left(\theta_{2}-a\right)^{2}}{(n+2)}$ & $\frac{n\left(\theta_{2}-a\right)^{2}}{(n+1)(n+2)}$ \\
\hline
\end{tabular}


Table 4. Tests of Hypotheses Related to the Parameter $\theta$ of $X \sim \operatorname{Uniform}\left(a, \theta_{2}\right)$

\begin{tabular}{|c|r|c|}
\hline$H_{0}: \theta=\theta_{0}$ & $H_{0}: \theta \leq \theta_{0}$ & $H_{0}: \theta \geq \theta_{0}$ \\
$H_{1}: \theta \neq \theta_{0}$ & $H_{1}: \theta>\theta_{0}$ & $H_{1}: \theta<\theta_{0}$ \\
\hline If $Y_{n} \geq y_{n U}$ or $Y_{n} \leq y_{n L}$ & If $Y_{n} \geq y_{n U} H_{0}$ is rejected & If $Y_{n} \leq y_{n L} H_{0}$ is rejected \\
$H_{0}$ is rejected & Don't reject $\mathrm{H}_{0}$ otherwise & Don't reject $\mathrm{H}_{0}$ otherwise \\
\hline Don't reject $\mathrm{H}_{0}$ otherwise & Where, & Where, \\
\hline Where, $y_{n L}=a+\left(\theta_{0}-a\right)\left(\frac{\alpha}{2}\right)^{1 / n}$ and & & $y_{n U}=a+\left(\theta_{0}-a\right)(1-\alpha)^{1 / n}$ \\
$y_{n U}=a+\left(\theta_{0}-a\right)\left(1-\frac{\alpha}{2}\right)^{1 / n}$ & & $y_{n L}=a+\left(\theta_{0}-a\right)(\alpha)^{1 / n}$ \\
\hline
\end{tabular}

If the results in (7.2) and (7.3) are substituted in (7.1)

$$
P\left(a+\left(\theta_{2}-a\right)\left(\frac{\alpha}{2}\right)^{1 / n}<Y_{n}<a+\left(\theta_{2}-a\right)\left(1-\frac{\alpha}{2}\right)^{1 / n}\right)=(1-\alpha) .
$$

Solving the above inequalities for $\theta$, the following Confidence Interval is obtained.

$$
P\left(a+\frac{Y_{n}-a}{\left(1-\frac{\alpha}{2}\right)^{1 / n}}<\theta_{2}<a+\frac{Y_{n}-a}{\left(\frac{\alpha}{2}\right)^{1 / n}}\right)=(1-\alpha)
$$

A $100(1-\alpha) \%$ confidence interval for $\theta_{2}$ is given as follows.

$$
\left(a+\frac{Y_{n}-a}{\left(1-\frac{\alpha}{2}\right)^{1 / n}}, a+\frac{Y_{n}-a}{\left(\frac{\alpha}{2}\right)^{1 / n}}\right)
$$

\section{Tests of Hypotheses Related to the}

\section{Parameter $\theta_{2}$ of the Distribution}

$$
X \sim \operatorname{Uniform}\left(a, \theta_{2}\right)
$$

If $H_{0}: \theta=\theta_{0}$ is to be tested against to any proper alternative hypothesis, a plausible test statistic is to be $Y_{n}=X_{M a x}$ for that the most efficient unbiased estimator is $W_{1}=\frac{(n+1) Y_{n}-a}{n}$, which is a linear function of $Y_{n}=X_{\text {Max }}$.

If the level of significance is chosen to be $\alpha$, then the decision rules, as given in the following table, are applicable.

\section{Conclusions}

For uniform distributions, of the types $X \sim \operatorname{Uniform}\left(\theta_{1}, b\right)$, and $X \sim \operatorname{Uniform}\left(a, \theta_{2}\right)$, some unbiased estimators for the unknown boundary values $\theta_{1}$ and $\theta_{2}$ are suggested. Among the suggest estimators, the most efficient estimator is selected. By the use the most efficient estimators, confidence interval and tests of hypotheses procedures are established. 


\section{Simulation Study}

To see the match between the established theoretical findings and the empirical results, a simulation study on a uniform distribution over the interval $\left(\theta_{1}=5, \theta_{2}=10\right)$ is carried out. 100 independent samples of size 100 are drawn from this uniform distribution. From each sample the first order statistic $Y_{1}$, the last order statistic $Y_{n}$, sample mean $\bar{X}$, sample medians $M(n$ is even) (for $\mathrm{n}=100)$, and $M(n$ is odd $)$ for $\mathrm{n}=99)$ are computed. Unbiased estimator values are computed from each sample. Summary of the simulation results are given in the following Table 5.

Table 5. Simulation summaries

\begin{tabular}{|c|c|c|}
\hline & Mean & Variance \\
\hline Sample Mean $(\bar{X})$ & 7,502283 & 0,021271 \\
\hline $\operatorname{Min}\left(Y_{1}\right)$ & 5,053689 & 0,003032 \\
\hline $\operatorname{Max}\left(Y_{n}\right)$ & 9,955695 & 0,001421 \\
\hline Sample Median, M(n even) & 7,506121 & 0,061029 \\
\hline Sample Median, M( $\mathrm{n}$ odd $)$ & 7,501759 & 0,063832 \\
\hline $\mathrm{T} 1$ & 5,004226 & 0,003093 \\
\hline $\mathrm{T} 2$ & 5,525145 & 14,49897 \\
\hline $\mathrm{T} 3$ & 5,004565 & 0,085083 \\
\hline $\mathrm{T} 4$ & 5,003519 & 0,255329 \\
\hline $\mathrm{T} 5$ & 5,012243 & 0,244115 \\
\hline W1 & 10,42261 & 30,93163 \\
\hline W2 & 10,00525 & 0,00145 \\
\hline W3 & 10,00457 & 0,085083 \\
\hline W4 & 10,00352 & 0,255329 \\
\hline W5 & 10,01224 & 0,244115 \\
\hline
\end{tabular}

Based on a simulation of 100 independent samples of size $\mathrm{n}=100$ from a uniform distribution over the interval $\left(\theta_{1}=5, \theta_{2}=10\right) \quad, \quad$ summary statistics, $\bar{X}, Y_{1}, Y_{n}, M($ neven $)$, and $M($ nodd $) \quad$ of $\quad 100$ observations are obtained.

Values of five different estimators for $\theta_{1}$, namely T1, T2, T3, T4, and T5 are computed from each sample by the use of proper summary statistics. The second and third columns of Table 5 contain the computed means and the variances of each summary statistics and of estimate values for $\theta_{1}$.

From Table 5, we observe that T1, T2, T3, and T5 are unbiased estimators of $\theta_{1}$. Among these unbiased estimators $\mathrm{T} 1$ is the best (the most efficient) estimator, for that it has the smallest variance.

In the same line, values of five different estimators for $\theta_{2}$, namely W1, W2, W3, W4, and W5 are computed from each sample by the use of proper summary statistics.

From the second and the third columns of Table 5, we observe that $\mathrm{W} 1, \mathrm{~W} 2, \mathrm{~W} 3, \mathrm{~W} 4$ and $\mathrm{W} 5$ are unbiased estimators of $\theta_{2}$. Among these unbiased estimators W2 is the best estimator, for that it has the smallest variance.

By using the formula (2.5), a 95\% confidence interval is computed for $\theta_{1}$ from each sample. Exactly 95 out of 100 confidence intervals contained the parameter value 5 of $\theta_{1}$.

Similar procedures are followed for $\theta_{2}$. By the use of the formula (5.5), 95\% confidence intervals for $\theta_{2}$ are computed. 94 out of 100 confidence intervals contained the parameter value 10 of $\theta_{2}$.

Simulation study results are seen to be in accordance with the theoretical findings.

A Simulation Study on A Uniform Ditribution Over the Interval $(5,10)$

\begin{tabular}{|c|c|c|c|c|c|c|c|c|c|}
\hline Sample 1 & Sample 2 & Sample 3 & Sample 4 & Sample 5 & Sample 6 & Sample 7 & Sample 8 & Sample 9 & $\begin{array}{c}\text { Sample } \\
10\end{array}$ \\
\hline 6.76805 & 5.54758 & 8.71645 & 7.32947 & 6.26586 & 7.69478 & 8.62869 & 5.77136 & 9.49987 & 7.23937 \\
\hline 6.64879 & 9.24694 & 5.11766 & 9.21047 & 6.51333 & 7.24894 & 9.61452 & 5.0222 & 9.40268 & 8.33267 \\
\hline 6.18835 & 8.82961 & 5.65464 & 7.76501 & 6.00813 & 7.04285 & 5.98805 & 8.67661 & 6.39686 & 6.45668 \\
\hline 8.92565 & 8.61381 & 6.33152 & 5.77686 & 5.65716 & 9.11983 & 5.47428 & 6.81726 & 8.08379 & 5.80943 \\
\hline 9.27706 & 5.19226 & 9.14137 & 8.91613 & 5.50441 & 6.1482 & 9.15237 & 5.74133 & 5.70093 & 6.37832 \\
\hline 9.69805 & 7.447 & 5.27074 & 8.73209 & 9.52611 & 9.74396 & 8.92942 & 8.69419 & 8.11978 & 7.46212 \\
\hline 9.56789 & 9.11269 & 5.96345 & 9.34895 & 9.44353 & 8.04405 & 6.18012 & 7.64807 & 9.53177 & 7.13468 \\
\hline 7.44082 & 7.77596 & 5.3617 & 6.7841 & 6.32015 & 5.99846 & 8.07746 & 8.43545 & 9.36648 & 5.17614 \\
\hline 9.72813 & 9.11189 & 7.05884 & 6.63384 & 9.70685 & 8.54253 & 9.96564 & 9.85792 & 7.12269 & 8.9224 \\
\hline 7.46514 & 7.06914 & 9.44628 & 5.96065 & 8.5456 & 6.99726 & 7.58934 & 6.05904 & 7.17869 & 5.70564 \\
\hline 8.86561 & 5.7497 & 9.80677 & 6.44889 & 5.60188 & 6.28214 & 7.08043 & 8.17612 & 9.03821 & 6.99094 \\
\hline 5.64825 & 8.37879 & 9.60301 & 9.26988 & 5.2388 & 6.00553 & 6.61076 & 9.29307 & 5.87516 & 9.03501 \\
\hline 7.75676 & 6.23922 & 7.51729 & 8.14453 & 5.49739 & 9.02683 & 5.50518 & 5.32239 & 6.76773 & 6.67583 \\
\hline 8.03505 & 5.17559 & 8.4334 & 6.97945 & 7.26849 & 9.3344 & 7.00371 & 5.67347 & 6.34599 & 9.72847 \\
\hline 7.91383 & 5.82104 & 6.11075 & 8.0797 & 7.72618 & 5.98542 & 6.14096 & 9.38147 & 9.80645 & 6.04649 \\
\hline
\end{tabular}




\begin{tabular}{|c|c|c|c|c|c|c|c|c|c|}
\hline 5.27165 & 7.36576 & 9.04968 & 7.10611 & 5.02197 & 6.07066 & 9.12631 & 6.74326 & 7.08122 & 6.08235 \\
\hline 7.5777 & 9.18829 & 5.01803 & 5.2689 & 7.79532 & 5.91644 & 6.42044 & 8.48295 & 8.37441 & 7.84362 \\
\hline 8.67416 & 7.68485 & 9.87295 & 5.12895 & 8.99236 & 7.01728 & 7.97886 & 7.58623 & 6.07359 & 7.71055 \\
\hline 5.27403 & 5.27586 & 7.36725 & 6.06629 & 7.00971 & 7.9662 & 9.61821 & 5.2543 & 6.21058 & 8.19278 \\
\hline 7.0574 & 6.73858 & 7.3245 & 9.21873 & 7.81031 & 9.38243 & 7.71026 & 8.78718 & 9.44682 & 5.20389 \\
\hline 9.87328 & 8.86696 & 9.23279 & 9.24976 & 5.99653 & 8.43504 & 8.92682 & 5.74824 & 6.78444 & 6.5277 \\
\hline 7.16373 & 6.29781 & 8.06776 & 9.20781 & 8.69283 & 8.152 & 5.33519 & 7.59396 & 9.83634 & 6.81062 \\
\hline 5.57791 & 8.07136 & 9.18987 & 7.23794 & 9.60694 & 7.59491 & 7.82591 & 6.98435 & 6.19687 & 5.69536 \\
\hline 5.60227 & 6.57299 & 8.33378 & 9.75946 & 9.20069 & 6.46122 & 6.62164 & 9.47102 & 9.08496 & 8.82302 \\
\hline 7.92689 & 8.40137 & 8.25053 & 7.68828 & 6.22544 & 7.94492 & 7.92489 & 8.54114 & 6.19162 & 7.24041 \\
\hline 5.27507 & 6.7398 & 5.5761 & 7.31552 & 9.7343 & 6.7126 & 5.60909 & 9.00667 & 5.96167 & 8.85088 \\
\hline 5.23583 & 6.70567 & 6.15485 & 8.7925 & 9.4837 & 5.44594 & 6.9962 & 6.8876 & 7.89363 & 6.62394 \\
\hline 8.41925 & 5.45209 & 5.42727 & 6.77572 & 5.70472 & 8.80198 & 8.95848 & 9.03918 & 7.95832 & 5.53407 \\
\hline 6.42925 & 7.48792 & 6.29917 & 5.4144 & 8.12612 & 8.09951 & 8.49912 & 6.17323 & 7.83436 & 9.80579 \\
\hline 7.73869 & 5.0058 & 8.84326 & 8.7735 & 5.68494 & 9.73225 & 8.84631 & 8.54916 & 5.94561 & 5.7347 \\
\hline 9.05125 & 6.25505 & 6.88755 & 5.56231 & 6.21997 & 6.3683 & 8.07337 & 9.86432 & 9.2897 & 7.34417 \\
\hline 5.17675 & 5.41977 & 7.20424 & 6.90153 & 5.44206 & 8.83918 & 9.85975 & 8.25992 & 6.71686 & 6.21734 \\
\hline 8.53267 & 9.87429 & 6.23478 & 5.78054 & 7.99312 & 8.95462 & 7.59182 & 6.21742 & 5.45427 & 7.47042 \\
\hline 5.00688 & 5.11108 & 5.7408 & 5.67876 & 7.41248 & 6.71499 & 6.45063 & 5.04365 & 8.61655 & 9.66729 \\
\hline 6.55685 & 6.49044 & 5.53936 & 6.65629 & 6.14796 & 8.12447 & 6.35537 & 5.14893 & 7.84643 & 8.22793 \\
\hline 9.36141 & 7.79091 & 9.08092 & 8.26076 & 9.67676 & 7.07278 & 8.97439 & 8.51532 & 9.32552 & 5.68898 \\
\hline 9.25425 & 9.76699 & 6.01214 & 7.98605 & 7.08992 & 8.91317 & 5.99515 & 5.83984 & 9.94706 & 6.31333 \\
\hline 5.70783 & 8.29902 & 5.9022 & 6.96476 & 6.68713 & 8.66273 & 9.94635 & 5.76362 & 6.10951 & 9.43733 \\
\hline 5.09335 & 8.38556 & 6.65674 & 7.80429 & 8.94538 & 6.10271 & 5.29402 & 5.33342 & 9.79042 & 6.1095 \\
\hline 5.13942 & 6.21019 & 5.46105 & 6.39426 & 8.47895 & 6.67935 & 7.11159 & 5.19055 & 9.01288 & 8.00078 \\
\hline 9.58752 & 6.82679 & 8.83202 & 6.98508 & 9.38232 & 7.49847 & 7.80716 & 7.75974 & 5.75598 & 5.06975 \\
\hline 5.5504 & 5.11758 & 5.56772 & 9.0693 & 7.68216 & 9.3875 & 5.40705 & 8.41356 & 6.83629 & 5.95721 \\
\hline 9.71618 & 5.02069 & 7.66213 & 9.0033 & 9.966 & 7.78309 & 5.51729 & 9.07735 & 7.51673 & 5.17899 \\
\hline 9.03292 & 8.47602 & 8.83159 & 6.20887 & 5.47519 & 7.15126 & 8.86561 & 6.3694 & 8.66828 & 7.03376 \\
\hline 9.18015 & 7.62615 & 8.6341 & 8.45812 & 9.32312 & 7.55031 & 5.57368 & 8.06073 & 7.46861 & 8.53911 \\
\hline 5.79433 & 5.94207 & 5.24463 & 6.38624 & 5.97973 & 8.57801 & 9.05113 & 6.08959 & 6.99081 & 5.73397 \\
\hline 5.71019 & 8.28413 & 9.88012 & 7.85481 & 9.80912 & 8.95461 & 6.62755 & 7.00377 & 5.91353 & 8.6948 \\
\hline 6.90311 & 7.49966 & 8.89027 & 9.93639 & 5.57294 & 7.61461 & 9.62354 & 5.84489 & 6.86733 & 7.33916 \\
\hline 6.01093 & 9.88304 & 8.05946 & 7.993 & 7.72002 & 6.92338 & 9.15678 & 7.42699 & 5.98298 & 6.47172 \\
\hline 8.29117 & 5.24312 & 5.46054 & 6.17231 & 9.17399 & 7.52364 & 9.02832 & 9.30765 & 8.3826 & 9.01742 \\
\hline 7.21463 & 9.3925 & 5.50457 & 9.61379 & 8.59673 & 9.09117 & 9.92957 & 9.79773 & 9.06485 & 9.59982 \\
\hline 5.69122 & 5.14753 & 9.94336 & 7.83586 & 9.71874 & 7.21083 & 9.225 & 6.64117 & 5.16821 & 7.76513 \\
\hline 7.39651 & 6.56979 & 8.79823 & 9.2966 & 5.71389 & 5.44684 & 9.49124 & 8.38406 & 9.94748 & 7.52972 \\
\hline 6.21596 & 6.77723 & 5.91663 & 7.29684 & 9.18548 & 8.2596 & 7.60838 & 9.81333 & 9.8305 & 9.63577 \\
\hline 9.3906 & 5.99472 & 7.03362 & 9.1611 & 8.75165 & 7.10397 & 7.34638 & 6.99033 & 7.57368 & 5.04623 \\
\hline 7.59387 & 8.75147 & 7.98545 & 5.99185 & 6.43094 & 9.14706 & 5.94997 & 6.82112 & 5.62793 & 7.73832 \\
\hline 9.52858 & 8.41175 & 6.10377 & 5.98491 & 5.12037 & 7.84236 & 6.95173 & 8.25666 & 8.2186 & 7.19077 \\
\hline 8.45732 & 7.6749 & 9.07446 & 6.99203 & 5.66462 & 8.24431 & 6.9998 & 8.93805 & 6.90706 & 7.50859 \\
\hline 8.72879 & 7.28557 & 8.83935 & 9.4945 & 8.69578 & 6.51762 & 7.88476 & 9.19904 & 5.10719 & 6.49467 \\
\hline 9.24246 & 7.91863 & 9.83302 & 9.28537 & 8.35988 & 7.37135 & 6.33597 & 6.92714 & 6.14444 & 9.00411 \\
\hline 5.69165 & 9.39004 & 8.42245 & 5.71779 & 8.40625 & 7.5988 & 9.4064 & 5.49549 & 6.13181 & 9.79494 \\
\hline 5.10031 & 6.06409 & 6.50803 & 7.26941 & 6.18215 & 8.75021 & 9.68249 & 9.29264 & 8.51029 & 5.562 \\
\hline 9.58141 & 6.30627 & 6.53334 & 5.14218 & 9.72103 & 8.15898 & 8.96704 & 9.89307 & 5.02439 & 8.57001 \\
\hline 6.91086 & 9.94315 & 6.16404 & 9.90682 & 5.80761 & 8.60099 & 9.79025 & 8.48124 & 7.23672 & 6.01227 \\
\hline 6.20842 & 5.40688 & 6.97988 & 6.79329 & 5.48799 & 5.01892 & 5.09225 & 9.47962 & 5.92262 & 5.8503 \\
\hline 6.69155 & 6.27316 & 8.22989 & 6.74831 & 7.72835 & 6.54153 & 5.78193 & 6.57418 & 6.2449 & 8.86547 \\
\hline
\end{tabular}




\begin{tabular}{|c|c|c|c|c|c|c|c|c|c|c|}
\hline & 5.95697 & 7.76551 & 7.90296 & 5.70668 & 8.64946 & 6.7981 & 7.71549 & 9.18902 & 9.51723 & 8.56753 \\
\hline & 9.93577 & 7.31901 & 8.89254 & 9.80263 & 9.92121 & 9.0717 & 5.80995 & 7.82189 & 8.43855 & 6.16525 \\
\hline & 7.16884 & 8.66276 & 8.55543 & 9.54445 & 8.06204 & 5.80005 & 5.07901 & 9.15531 & 8.90324 & 5.8136 \\
\hline & 8.46283 & 5.81383 & 9.64685 & 7.83238 & 9.79694 & 8.36708 & 6.20064 & 8.65119 & 6.50112 & 9.1347 \\
\hline & 5.85851 & 5.1967 & 7.12017 & 9.59633 & 7.43785 & 6.95533 & 5.80334 & 7.97374 & 9.75599 & 5.10726 \\
\hline & 9.18657 & 7.76747 & 5.66971 & 7.1275 & 6.84958 & 6.32708 & 5.90291 & 9.85473 & 5.03995 & 8.88636 \\
\hline & 8.7727 & 7.62816 & 5.58336 & 5.07287 & 8.3718 & 9.00943 & 8.0842 & 8.00979 & 6.97971 & 9.09677 \\
\hline & 8.48492 & 5.44725 & 6.16708 & 7.75861 & 7.20011 & 9.89235 & 7.99181 & 6.27968 & 9.17832 & 6.34832 \\
\hline & 9.12548 & 6.98984 & 8.02112 & 5.29448 & 8.65417 & 8.72267 & 9.07287 & 5.78202 & 8.90594 & 5.90646 \\
\hline & 6.20461 & 7.66837 & 9.89989 & 6.41731 & 8.89462 & 9.99651 & 6.04392 & 8.50089 & 5.3136 & 7.92458 \\
\hline & 6.72527 & 9.93648 & 7.03262 & 9.20973 & 8.26263 & 8.82813 & 8.33493 & 6.17131 & 6.74357 & 8.9929 \\
\hline & 9.16813 & 8.13906 & 6.33033 & 5.85007 & 7.30853 & 8.72447 & 9.69952 & 8.5079 & 7.59089 & 8.88475 \\
\hline & 8.4747 & 8.93317 & 7.3922 & 9.21486 & 8.73052 & 8.6101 & 9.06945 & 7.69367 & 5.82813 & 8.1123 \\
\hline & 8.65024 & 7.42468 & 8.8861 & 7.14189 & 7.27935 & 5.62626 & 9.63668 & 5.03305 & 8.24671 & 6.01152 \\
\hline & 7.71479 & 9.56845 & 8.43817 & 5.03158 & 8.19117 & 7.78087 & 5.55678 & 5.18307 & 5.49367 & 7.93077 \\
\hline & 5.14045 & 9.44141 & 6.53968 & 6.21086 & 9.56274 & 5.98351 & 9.85797 & 9.22242 & 8.19219 & 6.14648 \\
\hline & 6.30059 & 8.23071 & 9.96084 & 7.94444 & 7.33013 & 5.96955 & 7.07558 & 5.87318 & 6.35972 & 6.69438 \\
\hline & 7.39434 & 6.87728 & 8.30998 & 8.72429 & 6.93195 & 7.12342 & 6.59687 & 8.71186 & 7.14615 & 5.98767 \\
\hline & 6.29302 & 8.54591 & 7.95542 & 7.19951 & 5.92623 & 7.49941 & 9.17125 & 9.70237 & 9.77916 & 5.34305 \\
\hline & 8.41369 & 6.80944 & 5.40538 & 6.58762 & 5.38286 & 5.31034 & 5.38019 & 7.93686 & 8.39109 & 6.9979 \\
\hline & 9.34088 & 6.46698 & 5.90569 & 6.69883 & 8.84291 & 9.55467 & 7.13152 & 8.05994 & 7.17584 & 9.91563 \\
\hline & 7.91869 & 8.61218 & 5.20752 & 6.4563 & 6.38802 & 7.02478 & 8.93665 & 7.92058 & 9.49651 & 5.49833 \\
\hline & 9.95304 & 5.34705 & 8.26138 & 9.7086 & 5.40095 & 8.32756 & 7.65721 & 9.7321 & 6.30201 & 7.19636 \\
\hline & 7.68484 & 7.0938 & 7.03305 & 8.78562 & 9.55082 & 7.50063 & 7.15353 & 6.99172 & 7.11238 & 8.31738 \\
\hline & 7.18694 & 8.22582 & 6.05332 & 6.0943 & 9.18 & 8.75612 & 6.87896 & 6.50369 & 8.14279 & 5.90053 \\
\hline & 8.58824 & 9.27673 & 8.77767 & 9.74731 & 8.75946 & 5.93974 & 8.71412 & 7.07464 & 7.71726 & 9.86675 \\
\hline & 7.22256 & 5.66701 & 8.33937 & 9.3796 & 6.18925 & 8.36269 & 7.69222 & 5.78746 & 8.99914 & 8.62064 \\
\hline & 9.40729 & 7.96766 & 5.84759 & 5.77524 & 7.07049 & 5.48801 & 7.94256 & 5.11724 & 6.69397 & 9.05276 \\
\hline & 7.25673 & 5.23763 & 7.03162 & 7.41641 & 9.60502 & 7.78779 & 6.78918 & 6.69548 & 7.71054 & 5.13194 \\
\hline & 8.16415 & 8.86426 & 5.86231 & 6.5266 & 8.75165 & 6.25948 & 7.0017 & 8.10976 & 5.87843 & 7.07088 \\
\hline & 5.4353 & 6.75598 & 9.11823 & 6.54247 & 8.57984 & 8.49609 & 7.58698 & 9.86311 & 6.55117 & 8.07977 \\
\hline & 8.84267 & 5.3995 & 7.89863 & 7.53318 & 9.24741 & 9.55117 & 8.30927 & 5.85934 & 9.0229 & 9.8471 \\
\hline & 9.49711 & 5.77565 & 9.10296 & 6.01004 & 5.19007 & 6.41281 & 9.38935 & 9.98965 & 8.14817 & 6.11669 \\
\hline & 7.88351 & 9.52388 & 8.76388 & 7.64352 & 6.53911 & 6.2043 & 5.95083 & 7.04166 & 8.8628 & 6.00255 \\
\hline Mean & 7.5514061 & 7.3037618 & 7.4410716 & 7.4855762 & 7.6169842 & 7.6131248 & 7.6230981 & 7.5693427 & 7.5438337 & 7.3012481 \\
\hline Min & 5.00688 & 5.0058 & 5.01803 & 5.03158 & 5.02197 & 5.01892 & 5.07901 & 5.0222 & 5.02439 & 5.04623 \\
\hline $\operatorname{Max}$ & 9.95304 & 9.94315 & 9.96084 & 9.93639 & 9.966 & 9.99651 & 9.96564 & 9.98965 & 9.94748 & 9.91563 \\
\hline$M($ even $)$ & 7.639355 & 7.39522 & 7.379725 & 7.283125 & 7.727265 & 7.606705 & 7.674715 & 7.790815 & 7.49267 & 7.193565 \\
\hline$M($ odd $)$ & 7.639355 & 7.39522 & 7.345875 & 7.253675 & 7.761835 & 7.606705 & 7.674715 & 7.871235 & 7.352665 & 7.193565 \\
\hline$T 1$ & 4.9569488 & 4.955858 & 4.9682103 & 4.9818958 & 4.9721897 & 4.9691092 & 5.0298001 & 4.972422 & 4.9746339 & 4.9966923 \\
\hline$T 2$ & 5.25704 & 4.25815 & 6.04484 & 3.57539 & 6.566 & 9.64751 & 6.52964 & 8.95465 & 4.69548 & 1.47863 \\
\hline$T 3$ & 5.1028122 & 4.6075236 & 4.8821432 & 4.9711524 & 5.2339684 & 5.2262496 & 5.2461962 & 5.1386854 & 5.0876674 & 4.6024962 \\
\hline$T 4$ & 5.27871 & 4.79044 & 4.69175 & 4.50735 & 5.52367 & 5.21341 & 5.34943 & 5.74247 & 4.70533 & 4.38713 \\
\hline$T 5$ & 5.27871 & 4.79044 & 4.75945 & 4.56625 & 5.45453 & 5.21341 & 5.34943 & 5.58163 & 4.98534 & 4.38713 \\
\hline$W 2$ & 10.0025704 & 9.9925815 & 10.0104484 & 9.9857539 & 10.01566 & 10.0464751 & 10.0152964 & 10.0395465 & 9.9969548 & 9.9647863 \\
\hline$w 1$ & 5.69488 & 5.5858 & 6.82103 & 8.18958 & 7.21897 & 6.91092 & 12.98001 & 7.2422 & 7.46339 & 9.66923 \\
\hline W3 & 10.1028122 & 9.6075236 & 9.8821432 & 9.9711524 & 10.2339684 & 10.2262496 & 10.2461962 & 10.1386854 & 10.0876674 & 9.6024962 \\
\hline$W 4$ & 10.27871 & 9.79044 & 9.69175 & 9.50735 & 10.52367 & 10.21341 & 10.34943 & 10.74247 & 9.70533 & 9.38713 \\
\hline$w 5$ & 10.27871 & 9.79044 & 9.75945 & 9.56625 & 10.45453 & 10.21341 & 10.34943 & 10.58163 & 9.98534 & 9.38713 \\
\hline $\begin{array}{l}L C L \text { for } \\
L B\end{array}$ & 4.819250382 & 4.818129798 & 4.830819372 & 4.844878549 & 4.834907428 & 4.831742816 & 4.894090856 & 4.835146071 & 4.837418366 & 4.860079061 \\
\hline
\end{tabular}




\begin{tabular}{|c|c|c|c|c|c|c|c|c|c|c|}
\hline $\begin{array}{c}U C L \\
\text { for } L B \\
\end{array}$ & 5.005615692 & 5.004535418 & 5.016768515 & 5.030321946 & 5.020709513 & 5.01765874 & 5.077763956 & 5.020939571 & 5.023130125 & 5.044975655 \\
\hline $\begin{array}{c}L C L \text { for } \\
U B \\
\end{array}$ & 9.95429416 & 9.944401656 & 9.962096135 & 9.937639944 & 9.967257441 & 9.997775167 & 9.96689735 & 9.99091343 & 9.948732752 & 9.916874687 \\
\hline $\begin{array}{c}U C L \\
\text { for } U B\end{array}$ & 10.13916351 & 10.12890186 & 10.14725661 & 10.12188784 & 10.15261051 & 10.18426701 & 10.15223699 & 10.17714922 & 10.13339458 & 10.10034773 \\
\hline
\end{tabular}

A Simulation Study on A Uniform Ditribution Over the Interval (5,10) (continued)

\begin{tabular}{|c|c|c|c|c|c|c|c|c|c|}
\hline $\begin{array}{r}\text { Sample } \\
11\end{array}$ & Sample 12 & Sample 13 & Sample 14 & Sample 15 & Sample 16 & Sample 17 & Sample 18 & Sample 19 & Sample 20 \\
\hline 7.39785 & 6.20763 & 7.25944 & 9.16618 & 9.736 & 7.62824 & 9.22624 & 5.55963 & 5.08188 & 5.26447 \\
\hline 7.94401 & 5.69129 & 7.50581 & 8.4911 & 9.7161 & 5.81821 & 6.55312 & 6.8276 & 6.4105 & 5.84608 \\
\hline 7.06894 & 6.13494 & 8.63681 & 8.31787 & 9.2237 & 6.7997 & 8.20013 & 7.32031 & 5.91184 & 6.74863 \\
\hline 7.57285 & 9.33397 & 5.82982 & 9.98078 & 7.7035 & 8.83509 & 6.88446 & 9.09341 & 7.54447 & 6.9885 \\
\hline 6.8369 & 6.91514 & 6.94853 & 8.68123 & 7.8618 & 6.14139 & 5.85179 & 6.44702 & 9.17696 & 7.19409 \\
\hline 8.35096 & 5.78391 & 8.38171 & 5.26161 & 8.4084 & 9.44126 & 5.92579 & 9.7605 & 9.8743 & 6.69552 \\
\hline 7.50176 & 8.27563 & 9.6654 & 9.23336 & 5.0226 & 5.61973 & 7.416 & 6.37405 & 8.16673 & 9.13798 \\
\hline 8.39866 & 8.39755 & 5.27332 & 5.64921 & 7.5933 & 5.58701 & 8.18447 & 6.0201 & 9.40008 & 5.74566 \\
\hline 6.99428 & 9.86785 & 5.83636 & 7.22776 & 6.3804 & 5.97779 & 9.33531 & 9.44215 & 6.2974 & 7.6175 \\
\hline 9.99918 & 6.54547 & 9.38957 & 7.7293 & 8.943 & 5.09222 & 8.41037 & 6.13657 & 5.47198 & 6.15335 \\
\hline 7.6526 & 7.64054 & 7.09923 & 5.3689 & 6.4189 & 7.78039 & 7.83215 & 7.2793 & 5.94635 & 9.80417 \\
\hline 5.494 & 7.74346 & 8.93599 & 5.6543 & 9.0726 & 5.41968 & 8.61613 & 6.90328 & 6.73894 & 7.67689 \\
\hline 7.91504 & 5.53583 & 7.62648 & 7.04771 & 8.3337 & 6.16025 & 7.40354 & 7.1197 & 8.80171 & 8.36973 \\
\hline 5.07712 & 7.69366 & 8.5506 & 5.84612 & 8.9331 & 8.12448 & 9.4728 & 9.04545 & 5.75443 & 7.29942 \\
\hline 7.23817 & 6.42892 & 6.04477 & 7.41749 & 8.7025 & 6.53184 & 5.26031 & 6.27699 & 6.05236 & 7.35986 \\
\hline 7.85828 & 7.3348 & 6.84577 & 9.4722 & 5.6618 & 5.55453 & 9.36014 & 7.49751 & 7.64806 & 7.32806 \\
\hline 7.36382 & 7.52933 & 5.97317 & 7.69992 & 9.4438 & 9.06078 & 5.07384 & 6.3336 & 5.74785 & 8.92414 \\
\hline 8.06353 & 5.43307 & 9.85969 & 5.32654 & 7.3743 & 7.48679 & 5.44947 & 8.89453 & 6.38419 & 6.05716 \\
\hline 6.70263 & 8.26209 & 8.86738 & 6.79479 & 5.3812 & 5.87593 & 8.76083 & 5.54261 & 5.43776 & 8.72389 \\
\hline 9.04834 & 9.53827 & 7.7689 & 9.47768 & 8.397 & 6.64335 & 6.27355 & 6.19124 & 8.96379 & 6.41558 \\
\hline 6.36979 & 5.64731 & 8.53807 & 7.15427 & 6.9409 & 8.11397 & 7.56165 & 5.23458 & 6.63205 & 5.37156 \\
\hline 6.92077 & 9.54783 & 8.50996 & 7.55985 & 7.0424 & 8.24892 & 6.18634 & 6.22211 & 8.88804 & 8.69459 \\
\hline 5.53717 & 7.53904 & 7.70199 & 5.07621 & 9.8026 & 8.84672 & 6.3397 & 9.15922 & 9.13591 & 7.05403 \\
\hline 9.40802 & 6.04497 & 9.09425 & 7.09306 & 9.4289 & 5.23032 & 7.42046 & 8.00369 & 7.64426 & 6.63196 \\
\hline 5.06715 & 8.48232 & 9.98652 & 5.22271 & 8.2933 & 9.3684 & 7.02774 & 7.83541 & 8.26621 & 6.79833 \\
\hline 7.4118 & 9.02372 & 6.62458 & 7.81491 & 6.9305 & 8.16785 & 8.04377 & 7.17165 & 7.08211 & 7.78966 \\
\hline 9.99531 & 8.48427 & 8.45951 & 8.10252 & 7.6984 & 9.73513 & 7.26051 & 9.52155 & 7.63931 & 7.27974 \\
\hline 7.31508 & 6.23923 & 6.37046 & 6.29922 & 7.6716 & 7.67403 & 7.88892 & 8.02092 & 8.41438 & 9.06916 \\
\hline 6.67168 & 8.03332 & 5.23003 & 6.37034 & 5.9381 & 9.13418 & 8.22346 & 6.65097 & 9.16668 & 7.24098 \\
\hline 7.23515 & 5.9435 & 6.05655 & 8.4317 & 5.4536 & 6.23276 & 8.4368 & 9.56282 & 9.92996 & 5.52407 \\
\hline 6.51806 & 7.85592 & 5.33177 & 6.11586 & 8.1983 & 7.76704 & 8.73137 & 5.62925 & 6.65516 & 7.26843 \\
\hline 9.6771 & 9.82559 & 7.16112 & 8.128 & 9.2463 & 9.37293 & 8.17856 & 6.45708 & 5.79967 & 8.18539 \\
\hline 7.4341 & 8.74451 & 6.276 & 6.58604 & 5.6991 & 9.0135 & 6.82261 & 9.98926 & 7.97095 & 6.91902 \\
\hline 9.64631 & 8.92125 & 6.44299 & 5.29139 & 7.7926 & 8.18592 & 9.19378 & 9.35941 & 6.49807 & 9.36072 \\
\hline 5.29207 & 9.77339 & 6.03218 & 5.36048 & 5.6051 & 5.12744 & 8.19658 & 7.43825 & 5.10703 & 8.14276 \\
\hline 5.13412 & 8.19317 & 5.5289 & 7.38909 & 8.4656 & 5.3014 & 5.18899 & 7.05525 & 7.60794 & 8.6865 \\
\hline 6.75019 & 6.76985 & 5.88927 & 6.19036 & 5.6172 & 5.87572 & 5.82793 & 6.10128 & 9.87141 & 6.64686 \\
\hline 8.62868 & 7.84538 & 6.68348 & 5.76894 & 6.4758 & 9.24376 & 9.81656 & 7.8525 & 5.87902 & 8.25264 \\
\hline 9.93939 & 5.24889 & 7.7888 & 9.05191 & 6.17 & 7.16321 & 9.88333 & 6.04879 & 9.54144 & 9.32253 \\
\hline 9.28682 & 5.15208 & 5.71131 & 8.56557 & 9.2971 & 8.34593 & 5.52996 & 8.22105 & 5.72501 & 5.05149 \\
\hline 8.30137 & 8.35834 & 5.14256 & 7.73196 & 8.9883 & 5.19708 & 6.27565 & 5.27873 & 9.90038 & 9.36698 \\
\hline 9.59388 & 7.56078 & 8.47397 & 7.29452 & 8.9028 & 5.65104 & 7.5154 & 8.06941 & 6.37073 & 6.71419 \\
\hline 8.06009 & 6.38888 & 9.37852 & 6.01287 & 9.2642 & 6.26467 & 9.93527 & 5.13417 & 6.36996 & 5.92047 \\
\hline
\end{tabular}




\begin{tabular}{|c|c|c|c|c|c|c|c|c|c|}
\hline 8.51556 & 6.42493 & 6.23675 & 7.77947 & 8.5727 & 6.6845 & 5.60262 & 6.71558 & 8.53929 & 6.16471 \\
\hline 7.02528 & 5.1305 & 8.50583 & 5.33739 & 9.4496 & 8.6685 & 9.1879 & 5.15035 & 5.04674 & 6.08422 \\
\hline 8.86225 & 6.24247 & 9.80768 & 8.82819 & 6.1882 & 8.66858 & 8.57314 & 7.42402 & 6.37984 & 6.29987 \\
\hline 8.27566 & 7.0474 & 6.18524 & 7.24026 & 6.6582 & 7.32217 & 5.79064 & 9.77784 & 8.10408 & 8.81119 \\
\hline 9.93162 & 8.09323 & 8.91552 & 9.93509 & 8.6645 & 8.86669 & 6.34789 & 8.81902 & 5.46736 & 7.28297 \\
\hline 8.37572 & 5.61883 & 8.63409 & 5.99632 & 9.3585 & 8.32184 & 7.40013 & 7.18373 & 6.22218 & 7.1285 \\
\hline 8.65388 & 8.48995 & 9.34811 & 7.46079 & 5.4136 & 9.07994 & 8.38419 & 7.27919 & 8.81753 & 9.30363 \\
\hline 8.69014 & 8.20422 & 7.06179 & 5.12608 & 7.1828 & 5.56435 & 6.79376 & 7.74926 & 9.51067 & 9.80786 \\
\hline 9.53102 & 6.56193 & 7.58618 & 6.02701 & 6.6931 & 6.68482 & 5.44141 & 9.43515 & 8.79169 & 5.29862 \\
\hline 8.46926 & 5.40281 & 6.30829 & 7.31449 & 6.2906 & 9.01565 & 5.32804 & 9.7633 & 7.19075 & 8.51201 \\
\hline 5.34265 & 6.97902 & 5.36909 & 5.36397 & 5.0331 & 5.74077 & 8.18694 & 8.02474 & 6.70091 & 8.38229 \\
\hline 9.06678 & 6.98001 & 9.36352 & 5.81136 & 5.2576 & 8.11496 & 5.05098 & 9.06684 & 9.47276 & 5.63144 \\
\hline 9.23981 & 9.00532 & 7.21196 & 8.55793 & 6.5879 & 6.94823 & 9.46631 & 8.2726 & 6.20838 & 8.1244 \\
\hline 5.66355 & 6.4169 & 6.78388 & 6.58622 & 8.7434 & 9.90656 & 8.57594 & 5.19805 & 7.23249 & 7.75632 \\
\hline 7.22543 & 6.30703 & 7.30567 & 9.05109 & 9.9999 & 6.99865 & 8.70659 & 8.44717 & 9.91768 & 7.04789 \\
\hline 7.81943 & 5.0073 & 8.04645 & 5.68206 & 7.5186 & 5.87804 & 6.50219 & 7.57899 & 9.60546 & 6.60042 \\
\hline 8.93109 & 7.81565 & 7.13441 & 6.70723 & 5.4204 & 9.76292 & 8.91562 & 5.3944 & 8.32549 & 8.09718 \\
\hline 9.65457 & 8.73707 & 7.7928 & 5.64265 & 5.8887 & 6.09697 & 7.21258 & 6.42296 & 9.48355 & 5.27488 \\
\hline 8.5874 & 6.92289 & 8.07765 & 9.18122 & 7.552 & 6.21786 & 7.72036 & 5.08256 & 7.6745 & 5.29402 \\
\hline 8.29896 & 9.66623 & 6.01848 & 6.25046 & 8.7183 & 6.38391 & 5.65233 & 7.37934 & 6.69772 & 6.0828 \\
\hline 8.17236 & 5.2656 & 9.38141 & 5.34474 & 8.8397 & 5.92125 & 9.14714 & 7.48546 & 7.66778 & 8.18654 \\
\hline 8.83367 & 6.66726 & 8.27641 & 9.39273 & 8.0676 & 6.65641 & 6.95142 & 7.07142 & 6.10548 & 6.89464 \\
\hline 5.34083 & 6.6406 & 8.60935 & 7.92246 & 5.8396 & 9.05802 & 7.37061 & 8.07589 & 5.35419 & 5.64123 \\
\hline 8.0906 & 7.76244 & 9.72225 & 5.5272 & 6.9759 & 9.56182 & 5.56989 & 9.81599 & 8.53576 & 6.97032 \\
\hline 7.58749 & 9.66608 & 5.05439 & 7.15307 & 6.3469 & 9.56614 & 6.69554 & 7.47203 & 9.95491 & 9.11285 \\
\hline 6.7169 & 9.16153 & 7.13055 & 6.82499 & 7.4483 & 6.94713 & 8.70693 & 6.42977 & 8.92406 & 7.38939 \\
\hline 9.81761 & 9.68075 & 5.92115 & 9.92838 & 9.4644 & 9.09148 & 8.08565 & 5.02457 & 6.3539 & 6.19471 \\
\hline 9.01024 & 6.79721 & 9.46717 & 5.93763 & 9.9809 & 8.86075 & 8.73073 & 5.89269 & 7.81358 & 8.45515 \\
\hline 6.86641 & 8.20738 & 7.35658 & 8.13622 & 9.0001 & 7.34992 & 9.39392 & 7.37536 & 5.60076 & 8.03869 \\
\hline 7.35323 & 8.74021 & 7.61242 & 8.42627 & 9.7001 & 8.0541 & 6.56241 & 6.8519 & 7.36086 & 6.54893 \\
\hline 7.61803 & 6.14829 & 8.63496 & 8.8758 & 7.9314 & 9.60707 & 5.00066 & 7.58104 & 5.66064 & 9.93244 \\
\hline 5.57868 & 5.24022 & 5.72411 & 6.26774 & 8.5974 & 5.7903 & 7.58716 & 5.77288 & 5.09175 & 8.00952 \\
\hline 8.12611 & 8.39449 & 8.11326 & 6.8039 & 8.3532 & 7.67104 & 6.30606 & 5.45776 & 7.22413 & 6.9665 \\
\hline 5.75729 & 7.44862 & 7.50849 & 7.644 & 8.2103 & 7.36702 & 7.35778 & 7.11629 & 6.27391 & 6.1399 \\
\hline 7.2263 & 7.95143 & 5.89386 & 9.66861 & 5.9897 & 9.25666 & 7.40101 & 8.41466 & 8.2625 & 8.25507 \\
\hline 6.4719 & 8.81848 & 7.11683 & 7.84421 & 8.3013 & 5.77341 & 9.59417 & 6.11447 & 8.26719 & 8.2262 \\
\hline 8.25635 & 7.97871 & 8.26657 & 6.052 & 6.1524 & 5.90646 & 6.29579 & 7.28931 & 9.12862 & 9.76343 \\
\hline 7.51749 & 5.81842 & 7.451 & 8.28588 & 9.4695 & 6.95256 & 6.12368 & 5.36801 & 8.03506 & 6.80507 \\
\hline 6.8456 & 8.53818 & 8.42014 & 9.15292 & 5.9858 & 7.41112 & 7.8785 & 7.60355 & 9.95089 & 7.14803 \\
\hline 7.46963 & 6.60374 & 8.86763 & 6.0438 & 8.804 & 7.80519 & 7.81696 & 7.96352 & 7.8157 & 6.97771 \\
\hline 5.12147 & 7.4682 & 5.39092 & 6.26042 & 9.4348 & 7.26604 & 7.43905 & 9.63743 & 5.49536 & 8.62098 \\
\hline 5.90402 & 5.93774 & 9.24483 & 8.04131 & 7.4353 & 5.46798 & 8.953 & 9.56017 & 9.80873 & 8.75339 \\
\hline 7.26553 & 9.14071 & 6.7082 & 9.19207 & 5.0085 & 8.63621 & 9.71324 & 5.33261 & 6.608 & 6.61845 \\
\hline 6.69629 & 5.42626 & 7.93621 & 7.49468 & 6.562 & 8.36234 & 6.31127 & 7.08913 & 9.29109 & 6.91747 \\
\hline 6.22081 & 7.37053 & 8.67953 & 7.06509 & 7.426 & 8.90101 & 5.2426 & 6.2947 & 6.72996 & 7.27462 \\
\hline 9.69949 & 5.51725 & 9.0679 & 6.41517 & 8.6747 & 9.46768 & 7.47333 & 8.89542 & 7.17449 & 7.19151 \\
\hline 5.73183 & 6.3377 & 5.29299 & 7.00573 & 6.2077 & 9.07925 & 8.31147 & 9.63636 & 8.7862 & 8.12202 \\
\hline 6.81631 & 8.57068 & 6.78897 & 7.74131 & 5.1666 & 9.86332 & 7.72579 & 8.10177 & 8.54152 & 5.05227 \\
\hline 5.52755 & 9.28046 & 8.1645 & 6.69749 & 7.8452 & 5.53572 & 5.87355 & 6.78089 & 6.07446 & 5.74197 \\
\hline 6.62158 & 5.44138 & 7.23956 & 9.61705 & 7.1616 & 6.22685 & 6.72456 & 8.43433 & 9.84138 & 6.82374 \\
\hline 9.72252 & 8.27665 & 8.09126 & 6.84545 & 7.3639 & 7.77444 & 7.78668 & 5.32349 & 7.39328 & 9.89427 \\
\hline
\end{tabular}




\begin{tabular}{|c|c|c|c|c|c|c|c|c|c|}
\hline 5.32557 & 7.14012 & 7.89246 & 7.02957 & 8.7941 & 5.63638 & 7.73279 & 5.52783 & 8.55309 & 5.77849 \\
\hline 7.44546 & 7.66052 & 5.47908 & 6.17161 & 9.1419 & 6.64577 & 7.6545 & 6.74936 & 8.00272 & 5.11153 \\
\hline 7.89587 & 7.11172 & 8.53037 & 5.68772 & 7.6342 & 5.5546 & 8.43349 & 9.9478 & 5.4087 & 9.52389 \\
\hline 6.83903 & 5.14611 & 6.73316 & 7.30832 & 7.605 & 6.56172 & 5.58905 & 6.02784 & 6.02485 & 8.2161 \\
\hline 5.63146 & 5.39738 & 9.97097 & 9.10903 & 9.7046 & 8.55917 & 5.71887 & 7.70168 & 6.16477 & 7.02549 \\
\hline 8.32613 & 9.37987 & 7.02287 & 7.08365 & 8.4832 & 9.86445 & 8.88024 & 5.78705 & 8.95264 & 9.63638 \\
\hline 7.5828072 & 7.351035 & 7.4916724 & 7.2433333 & 7.675299 & 7.4377727 & 7.4395483 & 7.3125242 & 7.5052924 & 7.3758685 \\
\hline 5.06715 & 5.0073 & 5.05439 & 5.07621 & 5.0085 & 5.09222 & 5.00066 & 5.02457 & 5.04674 & 5.05149 \\
\hline 9.99918 & 9.86785 & 9.98652 & 9.98078 & 9.9999 & 9.90656 & 9.93527 & 9.98926 & 9.95491 & 9.93244 \\
\hline 7.509625 & 7.45841 & 7.50715 & 7.15367 & 7.74805 & 7.38907 & 7.494365 & 7.279245 & 7.576205 & 7.217535 \\
\hline 7.509625 & 7.45841 & 7.547335 & 7.15367 & 7.70095 & 7.35847 & 7.45619 & 7.284305 & 7.576205 & 7.217535 \\
\hline 5.0178215 & 4.957373 & 5.0049339 & 5.0269721 & 4.958585 & 5.0431422 & 4.9506666 & 4.9748157 & 4.9972074 & 5.0020049 \\
\hline 9.91718 & -3.34715 & 8.63852 & 8.05878 & 9.9899 & 0.56256 & 3.46227 & 8.91526 & 5.44591 & 3.17644 \\
\hline 5.1656144 & 4.70207 & 4.9833448 & 4.4866666 & 5.350598 & 4.8755454 & 4.8790966 & 4.6250484 & 5.0105848 & 4.751737 \\
\hline 5.01925 & 4.91682 & 5.09467 & 4.30734 & 5.4019 & 4.71694 & 4.91238 & 4.56861 & 5.15241 & 4.43507 \\
\hline 5.01925 & 4.91682 & 5.0143 & 4.30734 & 5.4961 & 4.77814 & 4.98873 & 4.55849 & 5.15241 & 4.43507 \\
\hline 10.0491718 & 9.9165285 & 10.0363852 & 10.0305878 & 10.049899 & 9.9556256 & 9.9846227 & 10.0391526 & 10.0044591 & 9.9817644 \\
\hline 11.78215 & 5.7373 & 10.49339 & 12.69721 & 5.8585 & 14.31422 & 5.06666 & 7.48157 & 9.72074 & 10.20049 \\
\hline 10.1656144 & 9.70207 & 9.9833448 & 9.4866666 & 10.350598 & 9.8755454 & 9.8790966 & 9.6250484 & 10.0105848 & 9.751737 \\
\hline 10.01925 & 9.91682 & 10.09467 & 9.30734 & 10.4019 & 9.71694 & 9.91238 & 9.56861 & 10.15241 & 9.43507 \\
\hline 10.01925 & 9.91682 & 10.0143 & 9.30734 & 10.4961 & 9.77814 & 9.98873 & 9.55849 & 10.15241 & 9.43507 \\
\hline 4.881785185 & 4.819686164 & 4.868545695 & 4.891185639 & 4.820931257 & 4.907797257 & 4.812796649 & 4.83760513 & 4.860608226 & 4.86553672 \\
\hline 5.065900953 & 5.006035798 & 5.053137722 & 5.074963247 & 5.007236102 & 5.090977301 & 4.999394117 & 5.023310171 & 5.045485785 & 5.050236987 \\
\hline 10.00044584 & 9.869082589 & 9.987782637 & 9.982041184 & 10.00116603 & 9.907802391 & 9.93651966 & 9.990523331 & 9.956164633 & 9.933688944 \\
\hline 10.18703734 & 10.05077227 & 10.17390161 & 10.16794591 & 10.18778439 & 10.0909369 & 10.12072575 & 10.17674457 & 10.14110378 & 10.11778941 \\
\hline
\end{tabular}

A Simulation Study on A Uniform Ditribution Over the Interval $(5,10)$ (continued)

\begin{tabular}{|c|c|c|c|c|c|c|c|c|c|}
\hline Sample 21 & Sample 22 & Sample 23 & Sample 24 & Sample 25 & Sample 26 & Sample 27 & Sample 28 & Sample 29 & Sample 30 \\
\hline 8.32569 & 5.75325 & 6.13597 & 7.53555 & 5.11664 & 9.08224 & 9.99042 & 6.58396 & 7.01388 & 7.2635 \\
\hline 7.46212 & 5.33049 & 5.68595 & 7.10432 & 6.19317 & 5.77521 & 9.98285 & 5.6621 & 8.41163 & 6.76243 \\
\hline 9.59227 & 5.16567 & 6.52314 & 8.00479 & 5.28679 & 5.8814 & 6.61661 & 6.21335 & 9.86478 & 8.85571 \\
\hline 8.39591 & 6.02589 & 8.67845 & 7.88271 & 8.92604 & 8.0815 & 7.0593 & 8.38374 & 7.44662 & 7.85309 \\
\hline 8.3823 & 9.62654 & 7.39604 & 6.10528 & 5.07929 & 6.00122 & 5.65181 & 7.54661 & 7.17612 & 7.47679 \\
\hline 5.7627 & 5.79353 & 7.57989 & 7.81238 & 5.0122 & 8.893 & 7.58773 & 5.40331 & 8.23479 & 7.57756 \\
\hline 7.60945 & 6.29223 & 6.09938 & 6.84769 & 5.15296 & 9.68669 & 6.75935 & 8.80203 & 5.16679 & 8.16733 \\
\hline 8.15443 & 6.53956 & 7.80029 & 8.98717 & 7.0657 & 9.57708 & 5.17218 & 7.92756 & 6.41966 & 7.34651 \\
\hline 6.91606 & 7.18033 & 8.8917 & 7.64828 & 5.33753 & 5.28144 & 8.62498 & 6.78056 & 5.21995 & 7.71554 \\
\hline 7.39946 & 6.8039 & 6.81733 & 6.9729 & 5.72004 & 5.59521 & 8.73899 & 9.45348 & 8.13322 & 8.72174 \\
\hline 6.37475 & 9.04566 & 6.50417 & 5.16776 & 5.63193 & 9.43085 & 8.14344 & 5.93248 & 6.97278 & 6.21404 \\
\hline 8.45752 & 6.48389 & 5.3978 & 9.86514 & 6.7095 & 6.21591 & 9.3044 & 8.95939 & 5.72177 & 9.72006 \\
\hline 6.53484 & 8.202 & 9.93562 & 9.32914 & 7.70935 & 7.23305 & 5.49412 & 5.76469 & 9.14226 & 7.42846 \\
\hline 5.16489 & 7.20354 & 7.53374 & 9.35638 & 6.59767 & 7.89046 & 6.77294 & 9.83523 & 7.40732 & 7.95444 \\
\hline 9.47808 & 6.22206 & 5.58332 & 9.59478 & 7.99221 & 6.43917 & 5.89302 & 9.99247 & 6.9696 & 9.40442 \\
\hline 9.23631 & 6.72295 & 8.34206 & 5.1359 & 6.68982 & 5.0244 & 6.02004 & 6.71983 & 6.41408 & 9.16651 \\
\hline 5.81773 & 9.97844 & 8.1384 & 8.93292 & 5.36105 & 6.86311 & 8.6027 & 7.84583 & 8.10285 & 6.17235 \\
\hline 9.45194 & 7.1549 & 6.49622 & 6.78082 & 7.38425 & 8.18952 & 8.76817 & 8.62527 & 7.40127 & 7.8021 \\
\hline 6.58326 & 7.84476 & 9.88987 & 6.47946 & 8.34488 & 9.66706 & 5.26086 & 8.0864 & 9.83865 & 5.92278 \\
\hline 7.37079 & 5.95003 & 7.18485 & 9.11627 & 6.98097 & 5.31315 & 8.45788 & 5.00991 & 5.44174 & 7.21229 \\
\hline 9.77103 & 6.43369 & 9.70458 & 5.54783 & 9.44731 & 7.05563 & 8.59299 & 5.8155 & 6.67589 & 5.6197 \\
\hline 9.92275 & 9.19744 & 9.79383 & 9.83196 & 9.80004 & 9.38907 & 8.59939 & 7.13723 & 8.11409 & 9.57908 \\
\hline 8.63247 & 6.82916 & 9.66182 & 6.18629 & 7.51857 & 6.00483 & 5.62462 & 8.37601 & 8.01362 & 6.6603 \\
\hline
\end{tabular}




\begin{tabular}{|c|c|c|c|c|c|c|c|c|c|}
\hline 7.85437 & 5.12673 & 7.61048 & 9.86566 & 6.05878 & 9.01645 & 8.80219 & 6.26343 & 7.85254 & 8.15468 \\
\hline 9.85044 & 8.78166 & 9.54995 & 9.11415 & 7.20682 & 8.13201 & 5.20044 & 6.22073 & 5.5691 & 7.24427 \\
\hline 8.35803 & 7.53815 & 7.9768 & 7.10602 & 6.23081 & 9.8711 & 5.00736 & 7.50726 & 5.88478 & 6.58255 \\
\hline 5.21047 & 5.74051 & 8.55309 & 9.48773 & 7.79278 & 8.48275 & 9.79649 & 5.33199 & 8.9342 & 8.24775 \\
\hline 8.8132 & 6.39609 & 5.44752 & 5.73028 & 9.52593 & 8.56755 & 7.04515 & 6.68785 & 8.18693 & 8.33047 \\
\hline 8.0482 & 8.69522 & 6.08674 & 9.55419 & 9.87047 & 9.34426 & 6.79469 & 5.27031 & 7.55688 & 9.12237 \\
\hline 9.4708 & 9.06963 & 8.42509 & 5.92478 & 5.52015 & 6.31504 & 6.41845 & 6.69683 & 7.66046 & 6.37926 \\
\hline 8.37135 & 6.00814 & 8.77829 & 7.92755 & 6.22218 & 7.69534 & 7.95063 & 7.22383 & 5.67456 & 5.48947 \\
\hline 9.35921 & 8.63166 & 7.10174 & 6.6094 & 7.27742 & 8.72676 & 6.90121 & 8.3384 & 8.44562 & 8.29378 \\
\hline 8.13584 & 8.66406 & 7.05679 & 6.99214 & 8.53729 & 7.11442 & 6.47795 & 5.38802 & 8.41168 & 5.82682 \\
\hline 9.70577 & 9.51162 & 6.79132 & 5.17175 & 6.7689 & 6.9018 & 5.32815 & 9.06795 & 9.07235 & 8.12531 \\
\hline 6.30491 & 6.89919 & 9.57747 & 7.65716 & 5.16844 & 7.19381 & 5.5668 & 9.33529 & 6.08724 & 6.84524 \\
\hline 6.84694 & 8.09432 & 8.32904 & 8.74449 & 8.89727 & 6.79455 & 5.153 & 7.97058 & 7.67589 & 7.95755 \\
\hline 9.42759 & 9.86337 & 7.76314 & 6.66112 & 5.57856 & 8.14185 & 9.19218 & 7.50049 & 8.70145 & 9.26928 \\
\hline 8.44692 & 9.38785 & 9.37161 & 6.63592 & 9.18742 & 7.59484 & 5.88403 & 7.24741 & 9.72363 & 5.62789 \\
\hline 6.37371 & 9.42335 & 9.34725 & 8.29872 & 9.85391 & 5.69642 & 5.69619 & 8.42181 & 7.74294 & 6.05066 \\
\hline 5.08498 & 8.52938 & 6.14002 & 7.36046 & 6.28566 & 8.81382 & 5.32175 & 7.98748 & 6.66847 & 8.60827 \\
\hline 9.2007 & 7.24265 & 6.98993 & 9.79685 & 6.47539 & 8.7651 & 8.62978 & 7.87737 & 8.08682 & 9.34668 \\
\hline 5.09686 & 6.75718 & 7.2148 & 6.28526 & 5.9538 & 7.2025 & 8.78464 & 7.52633 & 7.38987 & 9.86544 \\
\hline 6.48897 & 7.24311 & 5.67578 & 6.59609 & 7.08098 & 5.97434 & 8.39302 & 7.84144 & 5.43232 & 8.13497 \\
\hline 8.0231 & 9.37684 & 8.38881 & 9.14406 & 6.11128 & 7.31805 & 6.17675 & 8.30275 & 9.40497 & 5.23083 \\
\hline 9.55717 & 7.9431 & 7.27392 & 6.21884 & 6.5125 & 8.70587 & 9.80154 & 6.98046 & 9.82169 & 9.87062 \\
\hline 9.28342 & 8.84346 & 5.15286 & 9.65465 & 7.21903 & 7.85329 & 8.03664 & 6.97107 & 5.14758 & 7.83208 \\
\hline 8.50767 & 6.95314 & 7.45182 & 8.6744 & 7.61861 & 7.82214 & 5.48193 & 8.27924 & 6.72037 & 7.42882 \\
\hline 8.28484 & 8.80668 & 9.05997 & 5.92591 & 8.72074 & 7.43228 & 6.00952 & 5.312 & 9.66738 & 6.76058 \\
\hline 6.57427 & 6.89189 & 8.5574 & 5.78803 & 8.39316 & 7.54053 & 9.95541 & 6.19337 & 6.21624 & 8.43987 \\
\hline 8.70755 & 8.29218 & 9.46167 & 6.70254 & 9.69068 & 8.41251 & 6.43353 & 9.86517 & 9.9937 & 5.16463 \\
\hline 5.95035 & 9.11406 & 8.32017 & 5.18303 & 7.51086 & 8.16715 & 7.39361 & 8.7723 & 7.97123 & 5.4851 \\
\hline 9.47106 & 6.90114 & 6.62021 & 6.92079 & 7.82887 & 8.96076 & 7.76304 & 5.006 & 5.59473 & 5.74209 \\
\hline 5.21334 & 7.91109 & 5.90649 & 5.6749 & 7.61417 & 8.77656 & 9.42714 & 7.91209 & 5.91617 & 8.24382 \\
\hline 5.08345 & 6.73298 & 9.46502 & 5.59773 & 6.33785 & 8.03829 & 6.6409 & 8.52277 & 7.77127 & 8.0021 \\
\hline 9.93153 & 5.9156 & 7.58929 & 7.70522 & 5.58872 & 9.29319 & 9.13734 & 5.27659 & 5.24541 & 6.10749 \\
\hline 6.72426 & 8.7903 & 8.00611 & 9.48693 & 7.75885 & 9.44562 & 7.66871 & 9.4551 & 5.14162 & 8.62176 \\
\hline 8.70934 & 8.26347 & 5.99589 & 6.88425 & 6.02726 & 8.07495 & 9.88452 & 7.10123 & 9.46598 & 6.69237 \\
\hline 6.63213 & 5.32896 & 9.22016 & 9.79235 & 8.26484 & 7.65199 & 7.94584 & 5.68903 & 7.0186 & 5.45011 \\
\hline 8.57051 & 5.35863 & 5.85238 & 6.22104 & 6.38446 & 5.79889 & 5.44857 & 7.31848 & 8.71663 & 7.20317 \\
\hline 8.80074 & 5.34427 & 8.79146 & 5.5691 & 9.72043 & 5.81353 & 8.40854 & 5.6765 & 6.85618 & 8.19874 \\
\hline 6.59686 & 7.80487 & 9.63725 & 6.29436 & 8.02882 & 5.55195 & 8.87062 & 8.73813 & 8.89183 & 7.3207 \\
\hline 9.28042 & 7.09206 & 8.95505 & 8.63971 & 8.69459 & 8.34788 & 9.11057 & 5.93923 & 8.88901 & 6.43554 \\
\hline 7.4718 & 9.70124 & 7.71218 & 9.22338 & 7.42276 & 5.80467 & 6.80978 & 5.3872 & 6.14053 & 9.00922 \\
\hline 5.36482 & 6.39633 & 5.20002 & 9.13003 & 6.68784 & 7.53523 & 5.44916 & 7.43923 & 6.73776 & 6.59053 \\
\hline 9.2361 & 5.36731 & 8.49363 & 6.19681 & 8.7512 & 6.38547 & 6.70923 & 9.57723 & 5.93882 & 6.84534 \\
\hline 7.76462 & 7.72537 & 6.11152 & 7.43202 & 6.53933 & 8.44879 & 6.75255 & 9.52321 & 5.65836 & 6.57105 \\
\hline 9.554 & 6.96495 & 9.97953 & 9.11587 & 7.46974 & 9.20809 & 6.30623 & 9.30795 & 9.57838 & 7.02036 \\
\hline 5.90198 & 7.28968 & 8.73938 & 6.21479 & 8.55203 & 9.28329 & 6.29008 & 5.15888 & 8.02672 & 7.32017 \\
\hline 9.56169 & 9.6815 & 8.74877 & 5.26943 & 6.62279 & 7.01126 & 6.66614 & 6.16267 & 7.01844 & 7.79521 \\
\hline 7.74022 & 6.33242 & 9.29239 & 6.29484 & 5.76628 & 9.56238 & 8.53247 & 5.87328 & 7.33305 & 5.90003 \\
\hline 7.94114 & 8.28761 & 8.31355 & 7.24938 & 5.22979 & 5.48585 & 5.15516 & 8.16651 & 6.85857 & 8.33192 \\
\hline 5.00681 & 9.0607 & 6.82394 & 8.66481 & 6.99464 & 7.13776 & 7.79786 & 7.52859 & 9.36286 & 5.62292 \\
\hline 9.41515 & 7.35261 & 8.15893 & 9.64423 & 6.52943 & 5.29951 & 8.9449 & 5.88656 & 5.92009 & 8.80299 \\
\hline 7.68255 & 8.11154 & 5.51103 & 8.84758 & 8.84668 & 5.1523 & 8.89712 & 8.69061 & 8.56185 & 8.68377 \\
\hline
\end{tabular}




\begin{tabular}{|c|c|c|c|c|c|c|c|c|c|}
\hline 9.16417 & 7.00771 & 5.0861 & 5.06758 & 5.69542 & 9.75588 & 7.57315 & 9.75201 & 6.54216 & 7.16394 \\
\hline 6.58181 & 5.37791 & 8.30916 & 6.1962 & 8.55578 & 9.70515 & 6.12634 & 9.11282 & 9.50384 & 8.09805 \\
\hline 6.88542 & 9.96593 & 9.59138 & 8.58082 & 9.87487 & 5.59857 & 5.64299 & 9.57104 & 7.14321 & 7.11006 \\
\hline 7.05921 & 6.86049 & 8.2182 & 6.85334 & 8.45328 & 5.07638 & 8.66818 & 9.81014 & 9.2333 & 5.44467 \\
\hline 9.348 & 9.26233 & 5.08559 & 6.75299 & 6.25372 & 8.38338 & 6.14941 & 9.83879 & 5.6177 & 6.8766 \\
\hline 6.15159 & 8.62186 & 5.55241 & 6.67253 & 8.33838 & 9.27209 & 9.85426 & 5.53322 & 8.97406 & 7.55892 \\
\hline 5.36697 & 7.70981 & 7.08366 & 6.84166 & 9.12528 & 7.79355 & 5.44239 & 9.11704 & 7.02016 & 5.17327 \\
\hline 5.67579 & 9.12153 & 5.957 & 6.52146 & 7.12082 & 8.13858 & 5.76206 & 8.2026 & 9.90825 & 6.42041 \\
\hline 8.26166 & 5.19902 & 9.58008 & 7.51785 & 7.92083 & 9.45899 & 7.30978 & 9.3532 & 8.51319 & 9.97358 \\
\hline 6.9554 & 7.96893 & 6.71263 & 5.93681 & 7.52358 & 9.4103 & 6.44313 & 5.71085 & 6.03751 & 7.08 \\
\hline 9.82566 & 7.733 & 7.61559 & 8.76653 & 6.35292 & 9.91858 & 6.72181 & 6.90514 & 5.38461 & 8.02552 \\
\hline 9.72762 & 6.63478 & 9.24999 & 6.13954 & 7.73999 & 8.36853 & 8.74962 & 6.49554 & 9.98583 & 8.80602 \\
\hline 8.043 & 9.03275 & 5.35129 & 7.17005 & 7.29054 & 8.46986 & 6.81885 & 8.00299 & 5.51275 & 9.64817 \\
\hline 6.92699 & 6.98176 & 6.86259 & 8.46919 & 5.85317 & 7.35454 & 9.39212 & 8.71494 & 5.65092 & 5.71094 \\
\hline 9.93362 & 5.78553 & 6.32483 & 8.88344 & 9.8555 & 5.20436 & 6.41424 & 8.26311 & 8.21389 & 9.79654 \\
\hline 7.76587 & 5.04796 & 8.08696 & 5.56381 & 9.6022 & 8.87437 & 6.83705 & 8.4564 & 7.94055 & 9.94701 \\
\hline 6.59597 & 5.90034 & 5.60437 & 5.94579 & 9.24471 & 6.03496 & 7.86729 & 5.6491 & 8.40195 & 8.91079 \\
\hline 5.94233 & 5.76951 & 5.31754 & 9.59859 & 7.35535 & 7.45189 & 8.1489 & 8.11002 & 5.15887 & 5.3046 \\
\hline 7.61879 & 6.59192 & 9.35701 & 6.82095 & 6.54584 & 9.11542 & 7.05358 & 8.74775 & 7.70798 & 7.67933 \\
\hline 6.145 & 9.31874 & 7.06257 & 8.60142 & 7.06093 & 8.81227 & 9.15257 & 8.27367 & 6.69281 & 6.72126 \\
\hline 8.71196 & 9.84293 & 6.83098 & 6.25536 & 6.61502 & 9.38405 & 8.17532 & 8.17673 & 9.06596 & 6.21218 \\
\hline 9.51708 & 7.98002 & 9.44205 & 5.99122 & 6.21299 & 5.01264 & 8.36691 & 7.75685 & 7.50646 & 7.32819 \\
\hline 6.61961 & 7.94988 & 5.00158 & 8.55421 & 7.88629 & 7.11178 & 8.58084 & 6.90012 & 5.48261 & 7.49717 \\
\hline 9.17986 & 5.09501 & 8.62308 & 6.22316 & 6.98922 & 6.48201 & 7.70858 & 5.7019 & 5.69332 & 6.62124 \\
\hline 9.49229 & 5.3171 & 9.72923 & 6.56501 & 9.68348 & 5.50995 & 5.32583 & 5.07636 & 9.15882 & 9.05931 \\
\hline 9.90961 & 6.35248 & 7.7217 & 5.64547 & 7.99413 & 5.77287 & 7.50229 & 6.77848 & 5.30039 & 6.16587 \\
\hline 7.8319418 & 7.4174899 & 7.6170964 & 7.3924143 & 7.3113334 & 7.6170689 & 7.3575578 & 7.4658598 & 7.4299821 & 7.4715701 \\
\hline 5.00681 & 5.04796 & 5.00158 & 5.06758 & 5.0122 & 5.01264 & 5.00736 & 5.006 & 5.14162 & 5.16463 \\
\hline 9.93362 & 9.97844 & 9.97953 & 9.86566 & 9.87487 & 9.91858 & 9.99042 & 9.99247 & 9.9937 & 9.97358 \\
\hline 8.0456 & 7.223095 & 7.663885 & 6.98252 & 7.212925 & 7.807845 & 7.18454 & 7.5376 & 7.42697 & 7.42864 \\
\hline 8.03305 & 7.24288 & 7.663885 & 6.98252 & 7.212925 & 7.807845 & 7.05644 & 7.65173 & 7.47654 & 7.452805 \\
\hline 4.9568781 & 4.9984396 & 4.9515958 & 5.0182558 & 4.962322 & 4.9627664 & 4.9574336 & 4.95606 & 5.0930362 & 5.1162763 \\
\hline 3.29562 & 7.82244 & 7.93253 & -3.56834 & -2.63813 & 1.77658 & 9.03242 & 9.23947 & 9.3637 & 7.33158 \\
\hline 5.6638836 & 4.8349798 & 5.2341928 & 4.7848286 & 4.6226668 & 5.2341378 & 4.7151156 & 4.9317196 & 4.8599642 & 4.9431402 \\
\hline 6.0661 & 4.48576 & 5.32777 & 3.96504 & 4.42585 & 5.61569 & 4.11288 & 5.30346 & 4.95308 & 4.90561 \\
\hline 6.0912 & 4.44619 & 5.32777 & 3.96504 & 4.42585 & 5.61569 & 4.36908 & 5.0752 & 4.85394 & 4.85728 \\
\hline 9.9829562 & 10.0282244 & 10.0293253 & 9.9143166 & 9.9236187 & 9.9677658 & 10.0403242 & 10.0423947 & 10.043637 & 10.0233158 \\
\hline 5.68781 & 9.84396 & 5.15958 & 11.82558 & 6.2322 & 6.27664 & 5.74336 & 5.606 & 19.30362 & 21.62763 \\
\hline 10.6638836 & 9.8349798 & 10.2341928 & 9.7848286 & 9.6226668 & 10.2341378 & 9.7151156 & 9.9317196 & 9.8599642 & 9.9431402 \\
\hline 11.0661 & 9.48576 & 10.32777 & 8.96504 & 9.42585 & 10.61569 & 9.11288 & 10.30346 & 9.95308 & 9.90561 \\
\hline 11.0912 & 9.44619 & 10.32777 & 8.96504 & 9.42585 & 10.61569 & 9.36908 & 10.0752 & 9.85394 & 9.85728 \\
\hline 4.819177751 & 4.861874071 & 4.81375122 & 4.882231344 & 4.824770295 & 4.825226829 & 4.819748419 & 4.818337313 & 4.959053591 & 4.982928253 \\
\hline 5.005545674 & 5.046706093 & 5.00031435 & 5.066331061 & 5.010937039 & 5.01137715 & 5.006095813 & 5.004735469 & 5.140389809 & 5.163405635 \\
\hline 9.934869242 & 9.979700591 & 9.980790867 & 9.866892034 & 9.876104366 & 9.919825434 & 9.991683625 & 9.993734144 & 9.994964455 & 9.974839361 \\
\hline 10.11901375 & 10.16551798 & 10.16664894 & 10.04849997 & 10.05805606 & 10.10340858 & 10.17794816 & 10.18007519 & 10.18135141 & 10.16047535 \\
\hline
\end{tabular}

A Simulation Study on A Uniform Ditribution Over the Interval $(5,10)$ (continued)

\begin{tabular}{|c|c|c|c|c|c|c|c|c|c|}
\hline Sample 31 & Sample 32 & Sample 33 & Sample 34 & Sample 35 & Sample 36 & Sample 37 & Sample 38 & Sample 39 & Sample 40 \\
\hline 7.50035 & 5.24713 & 9.8954 & 8.80255 & 8.8218 & 6.59927 & 9.76463 & 6.64735 & 9.00522 & 8.29796 \\
\hline 9.39137 & 9.34341 & 9.58363 & 6.57885 & 7.122 & 5.22716 & 9.74086 & 5.31393 & 7.85267 & 9.30098 \\
\hline 5.10678 & 8.88935 & 6.40569 & 8.50099 & 6.08443 & 9.05548 & 5.31031 & 5.81083 & 8.48287 & 8.96436 \\
\hline
\end{tabular}




\begin{tabular}{|c|c|c|c|c|c|c|c|c|c|}
\hline 6.93517 & 6.03544 & 5.52368 & 5.7983 & 7.48132 & 7.5783 & 8.14952 & 7.92606 & 9.73019 & 7.86092 \\
\hline 8.43921 & 8.46033 & 5.95257 & 7.20855 & 9.91838 & 7.85581 & 7.56823 & 6.4932 & 5.23424 & 9.52892 \\
\hline 8.47548 & 9.28135 & 7.33526 & 6.60288 & 6.83983 & 9.78701 & 7.11568 & 9.57081 & 9.11385 & 6.90428 \\
\hline 8.6267 & 8.89018 & 5.46819 & 9.79767 & 7.40833 & 8.58843 & 5.92564 & 7.16664 & 8.66639 & 6.22338 \\
\hline 6.16086 & 6.53802 & 5.32322 & 6.88292 & 8.2209 & 9.24749 & 5.9092 & 6.47112 & 8.98785 & 8.95951 \\
\hline 6.34905 & 8.34066 & 5.07714 & 7.48032 & 8.28914 & 9.1217 & 7.68114 & 9.83668 & 9.82768 & 9.0615 \\
\hline 6.36305 & 9.86537 & 5.17876 & 7.57962 & 5.06465 & 9.04939 & 9.92067 & 5.20862 & 7.54751 & 9.08712 \\
\hline 5.26247 & 7.6131 & 9.12762 & 9.04396 & 6.90057 & 5.38525 & 6.12297 & 5.275 & 5.05169 & 7.04878 \\
\hline 9.43292 & 9.65217 & 9.90705 & 6.54693 & 6.74766 & 7.84515 & 6.64887 & 5.14738 & 5.68784 & 7.15085 \\
\hline 5.10103 & 8.12979 & 8.61176 & 7.15245 & 7.79231 & 7.44 & 5.01203 & 6.88381 & 9.1343 & 8.0252 \\
\hline 8.36157 & 5.95266 & 6.90353 & 7.47758 & 5.73901 & 5.96571 & 5.75286 & 7.6891 & 5.99593 & 5.8456 \\
\hline 6.91612 & 8.41876 & 8.81934 & 8.39008 & 6.29432 & 8.80102 & 7.25997 & 9.86909 & 8.03123 & 9.21003 \\
\hline 7.31561 & 9.99863 & 8.20564 & 6.68536 & 6.72675 & 9.26345 & 7.23124 & 7.84323 & 7.41001 & 5.29254 \\
\hline 7.88559 & 5.52625 & 9.87828 & 5.73659 & 7.30404 & 5.73118 & 6.21262 & 9.99663 & 9.97311 & 9.6172 \\
\hline 8.75016 & 5.59702 & 8.89966 & 7.08961 & 7.71737 & 9.8482 & 6.99923 & 8.34049 & 6.04299 & 9.34101 \\
\hline 5.46755 & 7.50067 & 7.87756 & 6.4981 & 5.93183 & 7.43826 & 8.91837 & 9.84959 & 5.44741 & 6.37042 \\
\hline 8.78039 & 5.12196 & 5.25938 & 6.31369 & 5.18612 & 7.30355 & 8.7283 & 9.75394 & 9.80027 & 9.02646 \\
\hline 8.9755 & 6.3974 & 9.40572 & 8.536 & 8.01396 & 6.13468 & 7.41033 & 5.45015 & 6.90211 & 7.75888 \\
\hline 9.52359 & 8.49771 & 8.20356 & 5.22742 & 5.96029 & 7.67899 & 5.15104 & 6.18517 & 9.49309 & 7.19216 \\
\hline 8.19882 & 6.89299 & 6.7108 & 8.98305 & 5.76676 & 6.04506 & 9.14157 & 5.87919 & 9.42711 & 5.82084 \\
\hline 6.98004 & 9.3487 & 8.95231 & 8.31513 & 6.53649 & 9.72419 & 7.06336 & 9.17046 & 8.38205 & 9.94284 \\
\hline 5.61322 & 8.9436 & 6.26396 & 6.98272 & 7.14582 & 5.89077 & 7.97074 & 8.60369 & 9.18188 & 5.06491 \\
\hline 9.79137 & 5.79373 & 5.07575 & 6.28582 & 8.05085 & 7.78088 & 5.45468 & 5.49343 & 5.83014 & 8.67372 \\
\hline 6.22033 & 6.80948 & 5.91316 & 8.39016 & 7.26063 & 7.66103 & 9.6192 & 7.43953 & 9.73846 & 7.72983 \\
\hline 9.39838 & 8.97061 & 9.88289 & 7.07068 & 5.07646 & 5.07269 & 8.97085 & 5.81337 & 7.77438 & 9.63095 \\
\hline 7.39763 & 9.88705 & 6.60971 & 7.49315 & 8.75298 & 6.87313 & 7.52418 & 5.70439 & 5.93907 & 5.13677 \\
\hline 6.95253 & 7.52177 & 6.72597 & 9.05985 & 6.7729 & 9.3604 & 9.25821 & 5.07968 & 8.20215 & 7.81762 \\
\hline 8.75217 & 5.73752 & 8.77873 & 5.80632 & 9.00841 & 5.34608 & 5.63368 & 6.90172 & 9.47084 & 9.39468 \\
\hline 5.34833 & 6.46865 & 6.24724 & 5.57895 & 5.10476 & 7.38875 & 6.62421 & 6.46137 & 9.82975 & 6.13191 \\
\hline 8.502 & 9.21032 & 8.34483 & 9.87609 & 7.86116 & 6.27031 & 8.40452 & 8.82969 & 8.37235 & 8.57947 \\
\hline 5.61407 & 6.29874 & 5.7808 & 6.74338 & 7.70448 & 5.18031 & 5.63428 & 6.31334 & 9.30713 & 6.57546 \\
\hline 7.18887 & 6.50371 & 9.72908 & 5.75651 & 6.47396 & 6.80489 & 9.56272 & 6.96724 & 7.1687 & 6.33079 \\
\hline 8.08008 & 7.1872 & 6.66952 & 9.22244 & 6.24408 & 7.25462 & 5.54531 & 6.24603 & 8.92379 & 6.49622 \\
\hline 9.39534 & 8.63983 & 6.09715 & 5.44615 & 5.95364 & 8.11025 & 7.1534 & 7.50291 & 9.77039 & 7.03312 \\
\hline 7.36883 & 5.60201 & 6.13864 & 6.41581 & 7.9705 & 8.26183 & 5.86545 & 8.36105 & 6.44638 & 8.18014 \\
\hline 5.03076 & 6.5941 & 7.11916 & 7.6769 & 7.25937 & 6.27984 & 9.9761 & 9.44285 & 6.98838 & 5.64943 \\
\hline 7.48446 & 6.41826 & 6.8072 & 6.57661 & 5.74379 & 9.63576 & 5.07302 & 9.98667 & 6.14888 & 6.64932 \\
\hline 6.60067 & 5.36763 & 9.22689 & 6.26972 & 7.76929 & 8.41126 & 9.79231 & 5.16177 & 7.73309 & 6.69538 \\
\hline 9.87631 & 6.52021 & 6.68256 & 9.3851 & 7.88302 & 6.40151 & 8.59067 & 6.01476 & 9.065 & 5.3806 \\
\hline 6.93997 & 5.16642 & 6.81449 & 5.61354 & 8.11308 & 7.27037 & 9.49548 & 9.7027 & 9.46306 & 9.92547 \\
\hline 8.97711 & 6.75733 & 6.65952 & 8.77258 & 7.10883 & 6.69459 & 9.8485 & 9.17647 & 6.92013 & 8.2229 \\
\hline 9.01033 & 8.63543 & 9.31001 & 9.80727 & 6.28439 & 8.81035 & 6.22439 & 8.46489 & 8.44683 & 7.22277 \\
\hline 6.95119 & 7.676 & 7.10224 & 6.56012 & 9.11109 & 7.5507 & 8.46004 & 7.77055 & 7.19512 & 6.10208 \\
\hline 8.27998 & 8.28741 & 9.90079 & 7.56961 & 9.82754 & 7.57486 & 5.40428 & 5.63512 & 5.67407 & 5.21846 \\
\hline 7.52858 & 9.9158 & 5.50121 & 9.11535 & 5.63298 & 7.76186 & 8.69459 & 6.93366 & 7.44959 & 5.25495 \\
\hline 6.92018 & 7.95367 & 8.1118 & 7.62766 & 8.54468 & 6.59354 & 6.16161 & 5.60092 & 8.87817 & 7.11485 \\
\hline 7.698 & 5.39568 & 6.82866 & 7.16574 & 7.262 & 5.77043 & 7.10444 & 9.56848 & 7.29469 & 6.63726 \\
\hline 5.49964 & 6.30695 & 7.14232 & 6.97931 & 7.68499 & 7.56297 & 5.24927 & 6.16807 & 8.19343 & 9.20254 \\
\hline 5.80615 & 7.97755 & 6.29881 & 6.7675 & 7.38663 & 9.62251 & 5.33776 & 6.10377 & 9.51476 & 5.79857 \\
\hline 8.42811 & 5.91292 & 5.96541 & 7.37976 & 6.52555 & 5.27592 & 9.75478 & 6.4022 & 7.16116 & 6.77476 \\
\hline 9.40245 & 6.98585 & 5.50676 & 5.32682 & 9.12917 & 6.7105 & 8.67354 & 9.2487 & 5.61845 & 9.51702 \\
\hline
\end{tabular}




\begin{tabular}{|c|c|c|c|c|c|c|c|c|c|}
\hline 5.10785 & 9.54979 & 6.37485 & 7.34524 & 5.01416 & 5.06697 & 5.22 & 6.52407 & 9.78722 & 6.35788 \\
\hline 5.79286 & 7.63631 & 7.14124 & 9.19054 & 5.44655 & 9.55481 & 8.31342 & 8.24977 & 8.58574 & 8.7989 \\
\hline 6.00535 & 6.79061 & 6.69654 & 8.05914 & 8.89574 & 8.87154 & 5.66521 & 7.83562 & 5.21879 & 7.30689 \\
\hline 8.30135 & 7.94624 & 8.25281 & 5.27925 & 9.90575 & 8.61063 & 8.65035 & 8.92262 & 7.94457 & 6.81333 \\
\hline 5.8929 & 9.94946 & 6.47397 & 5.687 & 9.5043 & 6.8656 & 9.74509 & 5.18851 & 7.28214 & 8.57533 \\
\hline 5.12307 & 9.03214 & 6.56241 & 8.694 & 7.54733 & 9.32573 & 7.08729 & 5.54121 & 5.28525 & 7.85561 \\
\hline 9.09673 & 9.20852 & 5.63572 & 8.50909 & 5.74765 & 6.94556 & 7.71247 & 9.27144 & 8.69491 & 6.41712 \\
\hline 7.09896 & 7.1381 & 9.65995 & 7.07506 & 6.56871 & 7.11553 & 7.68939 & 6.15319 & 5.38714 & 9.03766 \\
\hline 6.70307 & 7.81267 & 7.3581 & 7.23356 & 7.5603 & 9.30252 & 6.37883 & 7.29669 & 9.64474 & 6.46283 \\
\hline 5.74119 & 9.65478 & 9.60155 & 7.77059 & 5.55502 & 7.4182 & 6.27061 & 8.99578 & 5.59539 & 8.09088 \\
\hline 5.63535 & 7.51114 & 7.80895 & 9.50728 & 6.28914 & 7.02888 & 8.29441 & 9.4568 & 9.36215 & 6.2991 \\
\hline 8.14417 & 9.07637 & 7.75014 & 8.67529 & 7.9587 & 5.98642 & 8.26501 & 7.13449 & 9.49481 & 9.57277 \\
\hline 5.0483 & 7.24265 & 8.55692 & 9.59615 & 7.70646 & 7.28453 & 5.34746 & 5.0828 & 7.90193 & 9.21734 \\
\hline 9.04833 & 8.72591 & 7.5159 & 8.79805 & 8.30448 & 7.46856 & 6.83016 & 6.2488 & 7.98167 & 8.42516 \\
\hline 5.49696 & 7.65268 & 9.10518 & 5.49842 & 6.38216 & 7.12359 & 5.64852 & 6.74658 & 5.78611 & 8.29595 \\
\hline 6.90834 & 5.12963 & 6.64125 & 6.34092 & 7.73763 & 7.05909 & 8.96289 & 5.39704 & 8.93044 & 9.99625 \\
\hline 7.72702 & 7.28399 & 8.16153 & 9.91849 & 9.47267 & 6.17608 & 5.08349 & 6.52262 & 5.57163 & 8.30925 \\
\hline 9.48972 & 7.25995 & 7.32795 & 6.41024 & 6.46112 & 6.35974 & 6.09765 & 7.58295 & 7.9226 & 8.25202 \\
\hline 8.15677 & 7.71819 & 7.66333 & 6.31399 & 8.45629 & 6.38887 & 6.11225 & 5.10857 & 8.51196 & 8.93596 \\
\hline 9.43355 & 8.2281 & 5.77918 & 5.34246 & 7.68658 & 7.09204 & 7.95314 & 7.36108 & 8.06914 & 9.01574 \\
\hline 8.57609 & 6.20476 & 9.18717 & 5.87335 & 9.88776 & 7.97843 & 8.792 & 7.35794 & 9.63873 & 8.24092 \\
\hline 6.65405 & 8.65733 & 7.66848 & 7.85896 & 9.91895 & 9.34843 & 6.69237 & 9.96433 & 7.56576 & 9.12607 \\
\hline 9.53108 & 8.47913 & 5.83892 & 6.07332 & 5.35934 & 5.71528 & 9.78231 & 8.48638 & 7.70619 & 9.43349 \\
\hline 7.90557 & 7.61717 & 7.67768 & 8.91528 & 8.44884 & 6.30982 & 7.49963 & 7.61643 & 9.52309 & 5.12784 \\
\hline 8.60646 & 9.79069 & 9.70913 & 5.49767 & 7.58413 & 6.0019 & 8.24264 & 9.08876 & 9.14056 & 6.11842 \\
\hline 6.98536 & 5.44063 & 7.7044 & 8.87764 & 7.12364 & 8.91802 & 7.78517 & 8.29459 & 5.30002 & 7.69069 \\
\hline 6.0887 & 6.75514 & 9.3903 & 5.93204 & 5.18948 & 9.99587 & 5.19588 & 6.36054 & 6.35852 & 7.12462 \\
\hline 5.1886 & 5.64261 & 5.80165 & 8.53785 & 9.71369 & 9.85396 & 9.6512 & 5.81206 & 5.97774 & 6.56621 \\
\hline 6.31121 & 6.67925 & 9.34305 & 5.37089 & 7.89786 & 5.99516 & 5.58288 & 8.43911 & 9.35734 & 7.08135 \\
\hline 6.392 & 6.07415 & 6.96554 & 5.02307 & 5.53499 & 5.89981 & 9.12609 & 5.07862 & 8.29857 & 9.68776 \\
\hline 7.7541 & 8.46677 & 6.76784 & 9.39449 & 6.22619 & 8.45301 & 9.12479 & 8.46252 & 6.7461 & 8.73015 \\
\hline 6.48263 & 9.76504 & 5.36303 & 6.56167 & 6.16899 & 9.06327 & 7.78414 & 7.43133 & 8.82988 & 7.50803 \\
\hline 6.22499 & 7.84079 & 7.9608 & 5.02901 & 6.94961 & 8.33897 & 5.37405 & 9.62391 & 9.31231 & 8.01537 \\
\hline 6.63061 & 6.31841 & 9.77621 & 5.84552 & 7.26944 & 7.84017 & 8.09963 & 8.85667 & 9.23926 & 6.73126 \\
\hline 9.79488 & 6.1229 & 9.91444 & 5.83367 & 8.80545 & 6.28899 & 5.90027 & 7.79591 & 6.09259 & 8.51372 \\
\hline 8.85675 & 6.81318 & 7.85959 & 6.27168 & 9.31587 & 7.51534 & 9.88815 & 9.76111 & 9.39197 & 9.90941 \\
\hline 8.4955 & 7.61686 & 5.12982 & 9.27654 & 5.41725 & 9.66808 & 9.84191 & 5.55896 & 8.4537 & 8.91896 \\
\hline 5.83202 & 7.30084 & 8.99787 & 5.61518 & 5.8903 & 8.57491 & 9.3326 & 8.4068 & 7.92917 & 7.74168 \\
\hline 7.2049 & 7.77536 & 7.74656 & 9.77974 & 7.71381 & 6.25353 & 9.5249 & 9.76764 & 9.629 & 9.91017 \\
\hline 6.62744 & 7.4541 & 6.16705 & 8.05253 & 8.89099 & 5.8907 & 8.08662 & 8.02769 & 7.79993 & 7.12726 \\
\hline 7.70593 & 9.29906 & 6.04552 & 9.03362 & 6.75863 & 8.08085 & 7.18897 & 8.7436 & 6.15168 & 7.0943 \\
\hline 5.36637 & 8.00875 & 8.70935 & 5.40784 & 6.81813 & 9.39201 & 7.8435 & 7.44429 & 8.12506 & 8.24254 \\
\hline 6.95076 & 8.39484 & 5.6508 & 7.47934 & 5.93032 & 5.95169 & 7.09552 & 8.56172 & 9.65427 & 8.64521 \\
\hline 8.6586 & 7.77499 & 8.46304 & 9.12469 & 6.87499 & 5.61357 & 7.33172 & 8.18191 & 7.13528 & 5.85543 \\
\hline 6.30913 & 7.79716 & 5.62634 & 6.97933 & 8.79659 & 5.61594 & 7.56762 & 6.6486 & 5.80945 & 7.8774 \\
\hline 7.26166 & 7.84167 & 9.04309 & 5.98448 & 5.08924 & 7.77815 & 5.48356 & 8.0683 & 7.06436 & 8.12468 \\
\hline 7.3470365 & 7.5746329 & 7.4431615 & 7.3082438 & 7.2629051 & 7.4527839 & 7.4694661 & 7.4333784 & 7.8811669 & 7.731526 \\
\hline 5.03076 & 5.12196 & 5.07575 & 5.02307 & 5.01416 & 5.06697 & 5.01203 & 5.07862 & 5.05169 & 5.06491 \\
\hline 9.87631 & 9.99863 & 9.91444 & 9.91849 & 9.91895 & 9.99587 & 9.9761 & 9.99663 & 9.97311 & 9.99625 \\
\hline 7.23328 & 7.62674 & 7.235135 & 7.12103 & 7.261315 & 7.42823 & 7.511905 & 7.43543 & 8.050185 & 7.858265 \\
\hline 7.196885 & 7.62674 & 7.14178 & 7.12103 & 7.261315 & 7.42823 & 7.511905 & 7.43543 & 8.050185 & 7.836615 \\
\hline
\end{tabular}




\begin{tabular}{|c|c|c|c|c|c|c|c|c|c|}
\hline 4.9810676 & 5.0731796 & 5.0265075 & 4.9733007 & 4.9643016 & 5.0176397 & 4.9621503 & 5.0294062 & 5.0022069 & 5.0155591 \\
\hline-2.49269 & 9.86163 & 1.35844 & 1.76749 & 1.81395 & 9.58287 & 7.5861 & 9.65963 & 7.28411 & 9.62125 \\
\hline 4.694073 & 5.1492658 & 4.886323 & 4.6164876 & 4.5258102 & 4.9055678 & 4.9389322 & 4.8667568 & 5.7623338 & 5.463052 \\
\hline 4.39377 & 5.25348 & 4.28356 & 4.24206 & 4.52263 & 4.85646 & 5.02381 & 4.87086 & 6.10037 & 5.67323 \\
\hline 4.46656 & 5.25348 & 4.47027 & 4.24206 & 4.52263 & 4.85646 & 5.02381 & 4.87086 & 6.10037 & 5.71653 \\
\hline 9.9250731 & 10.0486163 & 9.9635844 & 9.9676749 & 9.9681395 & 10.0458287 & 10.025861 & 10.0465963 & 10.0228411 & 10.0462125 \\
\hline 8.10676 & 17.31796 & 12.65075 & 7.33007 & 6.43016 & 11.76397 & 6.21503 & 12.94062 & 10.22069 & 11.55591 \\
\hline 9.694073 & 10.1492658 & 9.886323 & 9.6164876 & 9.5258102 & 9.9055678 & 9.9389322 & 9.8667568 & 10.7623338 & 10.463052 \\
\hline 9.39377 & 10.25348 & 9.28356 & 9.24206 & 9.52263 & 9.85646 & 10.02381 & 9.87086 & 11.10037 & 10.67323 \\
\hline 9.46656 & 10.25348 & 9.47027 & 9.24206 & 9.52263 & 9.85646 & 10.02381 & 9.87086 & 11.10037 & 10.71653 \\
\hline 4.844027735 & 4.938654815 & 4.890708353 & 4.836048763 & 4.826803947 & 4.881598421 & 4.824593906 & 4.893686201 & 4.865744235 & 4.879461011 \\
\hline 5.029501738 & 5.120724831 & 5.07450313 & 5.021809791 & 5.012897535 & 5.065720907 & 5.010766996 & $\mathbf{5 . 0 7 7 3 7 3 8 5 7}$ & 5.050437038 & 5.063660385 \\
\hline 9.877544731 & 9.999895704 & 9.915684386 & 9.919735411 & 9.920195528 & 9.997135005 & 9.977359999 & 9.997895197 & 9.974369242 & 9.997515101 \\
\hline 10.05955018 & 10.18646667 & 10.09911301 & 10.1033152 & 10.10379249 & 10.18360296 & 10.16309005 & 10.18439152 & 10.15998769 & 10.18399724 \\
\hline
\end{tabular}

A Simulation Study on A Uniform Ditribution Over the Interval (5,10) (continued)

\begin{tabular}{|c|c|c|c|c|c|c|c|c|c|}
\hline Sample 41 & Sample 42 & Sample 43 & Sample 44 & Sample 45 & Sample 46 & Sample 47 & Sample 48 & Sample 49 & Sample 50 \\
\hline 7.43951 & 8.24635 & 6.9857 & 9.16292 & 6.89561 & 7.05886 & 7.51524 & 6.7313 & 5.95412 & 6.07004 \\
\hline 7.57102 & 6.56106 & 8.23664 & 7.00731 & 5.59381 & 8.01754 & 7.0963 & 7.57462 & 9.82414 & 6.72801 \\
\hline 6.77325 & 5.08852 & 7.98663 & 9.61054 & 8.49726 & 6.19844 & 9.21696 & 8.84272 & 5.92339 & 7.96327 \\
\hline 9.79135 & 8.41533 & 5.04852 & 9.38471 & 6.89829 & 9.90602 & 6.06974 & 5.90194 & 6.96043 & 5.49204 \\
\hline 6.57138 & 5.62135 & 6.74215 & 8.10656 & 5.95605 & 9.29961 & 5.79917 & 7.64465 & 7.42631 & 8.85193 \\
\hline 9.09014 & 5.55481 & 6.01842 & 7.29108 & 6.05688 & 5.97353 & 8.62974 & 6.39139 & 6.2272 & 5.3403 \\
\hline 5.51461 & 8.87642 & 5.60244 & 5.28958 & 5.46722 & 6.99885 & 5.37584 & 6.79277 & 6.13681 & 8.23701 \\
\hline 7.8468 & 8.89368 & 7.24821 & 8.91456 & 8.31677 & 7.62211 & 6.27116 & 9.86692 & 5.34224 & 5.56701 \\
\hline 8.6792 & 6.3491 & 5.62216 & 5.37401 & 6.01975 & 5.19198 & 8.29397 & 9.70763 & 5.75499 & 6.55636 \\
\hline 6.87539 & 7.61707 & 9.59302 & 8.56879 & 8.14354 & 7.35214 & 8.67519 & 8.25598 & 5.13631 & 9.40228 \\
\hline 6.18459 & 5.94701 & 5.5542 & 5.79957 & 8.90951 & 7.75874 & 5.79837 & 6.51989 & 9.80317 & 8.57684 \\
\hline 5.23315 & 7.54473 & 8.16906 & 9.75204 & 8.43854 & 5.53767 & 7.69683 & 8.41953 & 8.12961 & 5.312 \\
\hline 8.90708 & 7.6082 & 8.72935 & 6.24769 & 9.70217 & 8.3059 & 5.17356 & 7.97678 & 6.79428 & 5.3863 \\
\hline 5.83295 & 9.99723 & 9.49626 & 7.50843 & 9.83234 & 9.10314 & 6.51401 & 6.45117 & 7.75835 & 9.38284 \\
\hline 9.32006 & 9.63741 & 5.70256 & 8.53486 & 7.29334 & 7.78092 & 8.35213 & 9.38985 & 5.96788 & 7.80611 \\
\hline 9.3104 & 8.40734 & 7.62382 & 8.57436 & 7.67231 & 5.87987 & 5.76888 & 8.0549 & 9.74297 & 5.70827 \\
\hline 5.67176 & 7.42233 & 5.60536 & 9.96176 & 9.27612 & 6.77939 & 5.30697 & 7.36826 & 9.06778 & 8.97023 \\
\hline 9.79551 & 7.14915 & 6.50241 & 9.04957 & 8.81878 & 7.91764 & 5.65608 & 5.25155 & 5.74336 & 8.56599 \\
\hline 5.21052 & 5.88984 & 9.82599 & 5.37411 & 5.8502 & 8.07446 & 6.6818 & 8.20686 & 8.95578 & 8.86164 \\
\hline 5.9953 & 8.86643 & 9.24647 & 6.23347 & 8.56669 & 9.12914 & 5.33939 & 7.31624 & 7.48825 & 5.50116 \\
\hline 6.65791 & 6.78691 & 6.25997 & 6.60135 & 7.94366 & 7.50505 & 7.18998 & 7.8403 & 8.35399 & 9.19841 \\
\hline 8.56078 & 8.90699 & 6.64405 & 9.17714 & 7.94625 & 7.57455 & 7.17983 & 8.69869 & 6.37955 & 9.5397 \\
\hline 6.73062 & 5.20994 & 9.31602 & 9.05614 & 9.44758 & 9.03226 & 8.52391 & 7.94971 & 6.63326 & 5.89869 \\
\hline 9.41529 & 8.17317 & 7.07624 & 5.47399 & 7.36347 & 6.31873 & 8.94606 & 6.31982 & 9.9166 & 9.01191 \\
\hline 6.07554 & 9.62733 & 9.58128 & 6.70003 & 8.14372 & 8.76728 & 8.5526 & 5.69285 & 6.89756 & 9.84497 \\
\hline 8.01566 & 8.71194 & 6.23515 & 6.0835 & 9.74107 & 6.5938 & 5.55883 & 6.67754 & 5.19569 & 6.8125 \\
\hline 6.83475 & 9.07548 & 6.13443 & 5.9505 & 9.18959 & 5.89701 & 5.87925 & 6.99587 & 7.23445 & 8.32718 \\
\hline 7.9589 & 7.65341 & 7.72308 & 5.95331 & 7.29536 & 7.89416 & 9.46205 & 6.49586 & 8.3046 & 5.76099 \\
\hline 8.51384 & 5.63138 & 8.96095 & 9.78814 & 9.09645 & 6.34093 & 6.77067 & 7.38832 & 7.46259 & 8.61816 \\
\hline 8.92311 & 7.69951 & 6.51392 & 5.03922 & 5.66252 & 9.50021 & 9.15549 & 9.35 & 8.93735 & 7.82585 \\
\hline 5.1192 & 6.56533 & 9.03573 & 6.73994 & 6.30135 & 6.24742 & 8.20792 & 7.84861 & 9.00198 & 7.86626 \\
\hline 9.4041 & 5.73872 & 5.11939 & 7.01022 & 8.02802 & 7.16684 & 7.16019 & 8.01223 & 8.73716 & 7.42041 \\
\hline 9.94098 & 9.7951 & 6.69433 & 7.07338 & 8.9592 & 8.07521 & 6.66061 & 6.96828 & 7.05565 & 5.14813 \\
\hline 5.90571 & 7.03409 & 5.65142 & 8.92985 & 7.9998 & 8.93696 & 7.60167 & 5.83995 & 7.42758 & 9.59906 \\
\hline
\end{tabular}




\begin{tabular}{|c|c|c|c|c|c|c|c|c|c|}
\hline 5.38793 & 9.25165 & 5.04133 & 5.51345 & 8.69423 & 5.53007 & 7.197 & 5.49402 & 5.13618 & 7.88444 \\
\hline 9.07852 & 5.97285 & 9.82933 & 5.29632 & 6.7193 & 8.02162 & 9.6299 & 6.24232 & 9.40428 & 6.01784 \\
\hline 9.75015 & 8.81171 & 5.31653 & 6.94139 & 7.83926 & 8.14834 & 5.57836 & 9.22661 & 5.47605 & 9.75275 \\
\hline 6.76399 & 9.1946 & 9.32238 & 8.47283 & 5.35729 & 5.60662 & 7.7814 & 8.73219 & 7.61303 & 7.46602 \\
\hline 6.02291 & 5.11834 & 5.91528 & 9.12003 & 8.71052 & 9.90778 & 9.39268 & 5.95808 & 6.63507 & 6.41813 \\
\hline 5.25138 & 8.81288 & 5.9573 & 5.49332 & 6.87346 & 5.64291 & 5.07731 & 5.60835 & 9.47975 & 7.35195 \\
\hline 6.25045 & 7.1705 & 7.26168 & 6.15644 & 5.92243 & 8.75308 & 6.50225 & 5.93999 & 7.38841 & 5.44004 \\
\hline 7.33451 & 6.53746 & 9.63119 & 9.74086 & 6.03286 & 5.42392 & 8.05853 & 7.09627 & 8.34702 & 8.67129 \\
\hline 9.20082 & 7.83462 & 9.31918 & 9.26425 & 7.06266 & 8.93879 & 9.39374 & 9.63792 & 6.30204 & 8.00283 \\
\hline 9.97497 & 7.09639 & 7.92596 & 5.88119 & 6.85013 & 6.01892 & 6.99948 & 9.70543 & 7.45196 & 8.67672 \\
\hline 9.53287 & 9.41457 & 9.11055 & 8.86196 & 5.03096 & 6.46716 & 5.10722 & 7.29534 & 8.3244 & 7.7181 \\
\hline 5.84091 & 7.65869 & 5.44099 & 9.90768 & 7.47179 & 9.37263 & 6.66334 & 7.57088 & 7.95822 & 7.39396 \\
\hline 9.18119 & 7.21974 & 8.51952 & 5.89365 & 5.88786 & 6.52954 & 6.95884 & 5.76143 & 6.84519 & 9.2574 \\
\hline 6.96248 & 6.3832 & 9.70854 & 5.70285 & 5.24924 & 9.15932 & 7.35008 & 9.51421 & 5.76095 & 7.19165 \\
\hline 5.24717 & 6.48257 & 6.62552 & 8.78999 & 6.20273 & 5.52388 & 7.71603 & 5.84104 & 9.59808 & 6.978 \\
\hline 6.41804 & 9.61701 & 9.65573 & 6.44594 & 5.06977 & 8.21815 & 7.5542 & 5.66472 & 7.65665 & 8.02003 \\
\hline 5.66935 & 8.12661 & 7.20189 & 6.86661 & 6.76592 & 6.13275 & 8.78086 & 5.73257 & 6.02749 & 9.12578 \\
\hline 7.47293 & 5.9308 & 8.20636 & 5.09093 & 5.0075 & 7.94199 & 5.88725 & 5.21135 & 9.67117 & 9.2848 \\
\hline 6.57728 & 6.9568 & 9.94126 & 7.16476 & 9.93408 & 9.88816 & 5.27527 & 6.09702 & 9.18018 & 6.79695 \\
\hline 5.11535 & 7.28443 & 5.64122 & 9.61041 & 5.81708 & 9.63919 & 9.11772 & 8.69824 & 8.41551 & 6.03511 \\
\hline 9.5647 & 6.64926 & 6.33472 & 9.07005 & 8.39516 & 8.86788 & 7.67557 & 8.43853 & 9.04269 & 8.1958 \\
\hline 5.35054 & 6.61546 & 8.239 & 9.00267 & 6.06365 & 9.02672 & 8.31096 & 9.10896 & 7.50541 & 8.59496 \\
\hline 9.34019 & 8.65791 & 6.64103 & 9.51511 & 6.48266 & 7.0065 & 5.40455 & 7.42353 & 9.82435 & 7.5418 \\
\hline 6.95715 & 6.33275 & 9.39788 & 8.53616 & 7.71581 & 6.27857 & 6.87064 & 7.22672 & 8.38683 & 9.87279 \\
\hline 8.555 & 9.39175 & 9.05629 & 9.31881 & 6.08377 & 8.03252 & 8.79059 & 8.26784 & 9.44529 & 6.7277 \\
\hline 5.51522 & 8.65433 & 6.59797 & 7.79202 & 5.82398 & 7.00401 & 6.32255 & 6.61919 & 7.32542 & 7.63007 \\
\hline 6.98757 & 5.39166 & 6.97215 & 6.52214 & 9.80779 & 8.902 & 8.36381 & 7.18559 & 8.63179 & 7.93261 \\
\hline 8.72711 & 6.21024 & 7.63766 & 8.87125 & 8.26993 & 9.9263 & 7.644 & 7.96486 & 5.08055 & 6.36137 \\
\hline 9.9309 & 5.12694 & 6.95474 & 6.35751 & 9.14504 & 6.60382 & 6.32103 & 5.89882 & 5.65283 & 7.62126 \\
\hline 7.13662 & 9.22069 & 8.40073 & 8.81758 & 8.9091 & 6.74108 & 5.85272 & 5.13041 & 9.11331 & 5.09419 \\
\hline 8.51499 & 9.92393 & 8.20698 & 8.82897 & 8.11063 & 6.18418 & 8.03629 & 9.6953 & 9.97321 & 9.41404 \\
\hline 6.08532 & 9.06559 & 5.81364 & 6.48222 & 9.83561 & 7.95791 & 5.81131 & 9.81686 & 5.28384 & 5.26181 \\
\hline 7.9058 & 7.03432 & 5.36941 & 6.31882 & 6.13581 & 6.9675 & 6.07276 & 5.78281 & 8.53467 & 9.8859 \\
\hline 8.96789 & 7.67053 & 8.83847 & 7.64882 & 8.6043 & 9.35202 & 8.08925 & 6.17718 & 5.58029 & 6.45991 \\
\hline 8.22032 & 9.57666 & 8.98342 & 5.79114 & 7.02505 & 9.10283 & 5.18413 & 5.70371 & 6.85401 & 6.72302 \\
\hline 6.27195 & 8.22608 & 7.36932 & 5.56267 & 6.39519 & 6.76235 & 5.13912 & 5.18073 & 6.08412 & 8.43374 \\
\hline 9.96244 & 7.96592 & 5.40733 & 5.57619 & 6.68363 & 6.26084 & 7.68457 & 5.04036 & 9.17757 & 8.13467 \\
\hline 8.28219 & 6.41225 & 7.00406 & 9.32312 & 6.84285 & 9.86887 & 8.0585 & 7.24348 & 9.72408 & 7.39015 \\
\hline 5.88895 & 5.03994 & 6.60156 & 6.67636 & 6.82369 & 5.47815 & 7.0661 & 9.36929 & 6.5768 & 5.37066 \\
\hline 7.34311 & 9.75218 & 8.88316 & 5.26618 & 8.6049 & 6.27582 & 5.14785 & 8.36003 & 5.03886 & 8.05789 \\
\hline 8.97547 & 9.71587 & 9.3962 & 9.65488 & 5.55818 & 6.35421 & 7.37417 & 8.68283 & 6.32769 & 8.28047 \\
\hline 5.08764 & 8.62187 & 7.78397 & 5.70435 & 8.50117 & 8.23416 & 6.96361 & 8.50785 & 6.34794 & 5.05514 \\
\hline 8.34194 & 8.76304 & 9.21736 & 9.33399 & 9.68556 & 6.18819 & 6.93515 & 5.56871 & 8.41116 & 7.58874 \\
\hline 7.5373 & 7.68996 & 6.42551 & 8.80661 & 7.07111 & 5.92674 & 9.76143 & 8.65908 & 9.61686 & 7.11489 \\
\hline 7.94285 & 5.71637 & 7.79367 & 5.27705 & 9.51942 & 5.86369 & 5.65145 & 6.85014 & 8.1267 & 5.81606 \\
\hline 8.05711 & 7.86758 & 8.41685 & 7.60925 & 8.59745 & 5.57227 & 5.42199 & 9.42663 & 6.74462 & 9.74792 \\
\hline 9.82766 & 7.72996 & 8.79661 & 7.00486 & 5.18294 & 8.47841 & 7.12482 & 9.22974 & 7.83256 & 5.55829 \\
\hline 8.09716 & 7.31975 & 7.75975 & 7.13936 & 8.29423 & 9.9513 & 5.81243 & 7.28782 & 5.23318 & 9.08026 \\
\hline 8.61072 & 9.23133 & 5.7962 & 8.28836 & 5.00868 & 5.38611 & 7.28355 & 7.09029 & 5.32676 & 6.14384 \\
\hline 7.20674 & 7.78688 & 5.68589 & 9.00133 & 9.56598 & 7.97488 & 7.78226 & 5.05105 & 5.1623 & 9.97439 \\
\hline 7.7513 & 7.95667 & 5.4114 & 8.85065 & 7.28274 & 5.19199 & 5.75819 & 7.66748 & 5.3924 & 9.95346 \\
\hline
\end{tabular}




\begin{tabular}{|c|c|c|c|c|c|c|c|c|c|}
\hline 9.40413 & 8.94034 & 5.5852 & 9.99187 & 5.37086 & 6.81897 & 8.73733 & 7.86887 & 9.95678 & 9.62016 \\
\hline 5.77088 & 5.53858 & 6.27369 & 6.48469 & 6.09167 & 5.50229 & 9.99484 & 5.17116 & 5.11626 & 7.69289 \\
\hline 5.23131 & 5.44623 & 6.08715 & 6.07144 & 9.142 & 5.57742 & 7.46513 & 5.84182 & 9.05438 & 9.0399 \\
\hline 9.99467 & 9.89436 & 9.15455 & 8.05391 & 8.86884 & 9.12612 & 7.9147 & 9.11843 & 9.85325 & 9.4755 \\
\hline 9.19098 & 9.97195 & 8.06076 & 9.69721 & 5.42229 & 8.01647 & 9.3464 & 5.48021 & 9.51128 & 6.28591 \\
\hline 7.49843 & 5.13288 & 9.63548 & 6.36749 & 5.84569 & 7.47162 & 8.90698 & 6.34547 & 7.9542 & 9.87033 \\
\hline 7.50273 & 7.78533 & 5.61616 & 7.97225 & 9.77321 & 6.64285 & 6.17817 & 8.69537 & 5.60874 & 9.81662 \\
\hline 5.90043 & 9.26598 & 6.68498 & 7.95444 & 8.23315 & 5.50794 & 8.61171 & 5.0466 & 5.76493 & 9.56114 \\
\hline 6.11802 & 7.16928 & 7.13987 & 6.7704 & 6.07435 & 6.71233 & 6.38886 & 9.68132 & 6.73007 & 9.03472 \\
\hline 7.25812 & 6.67925 & 7.27082 & 6.39514 & 8.22222 & 8.51572 & 8.83161 & 9.69489 & 5.34264 & 8.60396 \\
\hline 8.34532 & 5.19177 & 6.70078 & 8.97641 & 8.99011 & 7.90163 & 8.92595 & 5.87858 & 8.58469 & 6.57678 \\
\hline 7.4234 & 5.35588 & 7.41259 & 7.92591 & 7.30127 & 9.71997 & 6.73075 & 7.7946 & 5.51657 & 5.25574 \\
\hline 5.36923 & 8.01188 & 7.1629 & 6.66586 & 5.49855 & 6.34009 & 7.96571 & 5.30967 & 5.05268 & 6.83756 \\
\hline 6.10584 & 7.90877 & 8.89931 & 8.93062 & 5.34218 & 7.85024 & 8.8389 & 9.78126 & 9.30543 & 8.08921 \\
\hline 8.46396 & 8.70763 & 9.60679 & 8.30677 & 6.70285 & 9.51303 & 8.00947 & 9.86259 & 6.94866 & 8.39986 \\
\hline 7.5122486 & 7.5949586 & 7.4454103 & 7.5605038 & 7.4072754 & 7.4553969 & 7.2466371 & 7.3699762 & 7.4200566 & 7.6781372 \\
\hline 5.08764 & 5.03994 & 5.04133 & 5.03922 & 5.0075 & 5.19198 & 5.07731 & 5.04036 & 5.03886 & 5.05514 \\
\hline 9.99467 & 9.99723 & 9.94126 & 9.99187 & 9.93408 & 9.9513 & 9.99484 & 9.86692 & 9.97321 & 9.97439 \\
\hline 7.48568 & 7.680245 & 7.254945 & 7.55884 & 7.298315 & 7.488335 & 7.19349 & 7.30579 & 7.426945 & 7.846055 \\
\hline 7.48568 & 7.66461 & 7.254945 & 7.399755 & 7.33237 & 7.488335 & 7.184905 & 7.30579 & 7.43977 & 7.846055 \\
\hline 5.0385164 & 4.9903394 & 4.9917433 & 4.9896122 & 4.957575 & 5.1438998 & 5.0280831 & 4.9907636 & 4.9892486 & 5.0056914 \\
\hline 9.46167 & 9.72023 & 4.06726 & 9.17887 & 3.34208 & 5.0813 & 9.47884 & -3.44108 & 7.29421 & 7.41339 \\
\hline 5.0244972 & 5.1899172 & 4.8908206 & 5.1210076 & 4.8145508 & 4.9107938 & 4.4932742 & 4.7399524 & 4.8401132 & 5.3562744 \\
\hline 4.97136 & 5.32922 & 4.50989 & 4.79951 & 4.66474 & 4.97667 & 4.36981 & 4.61158 & 4.87954 & 5.69211 \\
\hline 4.97136 & 5.36049 & 4.50989 & 5.11768 & 4.59663 & 4.97667 & 4.38698 & 4.61158 & 4.85389 & 5.69211 \\
\hline 10.0446167 & 10.0472023 & 9.9906726 & 10.0417887 & 9.9834208 & 10.000813 & 10.0447884 & 9.9155892 & 10.0229421 & 10.0241339 \\
\hline 13.85164 & 9.03394 & 9.17433 & 8.96122 & 5.7575 & 24.38998 & 12.80831 & 9.07636 & 8.92486 & 10.56914 \\
\hline 10.0244972 & 10.1899172 & 9.8908206 & 10.1210076 & 9.8145508 & 9.9107938 & 9.4932742 & 9.7399524 & 9.8401132 & 10.3562744 \\
\hline 9.97136 & 10.32922 & 9.50989 & 9.79951 & 9.66474 & 9.97667 & 9.36981 & 9.61158 & 9.87954 & 10.69211 \\
\hline 9.97136 & 10.36049 & 9.50989 & 10.11768 & 9.59663 & 9.97667 & 9.38698 & 9.61158 & 9.85389 & 10.69211 \\
\hline 4.903045151 & 4.853552698 & 4.854994931 & 4.852805642 & 4.81989368 & 5.011306001 & 4.892326974 & 4.853988481 & 4.852432114 & 4.869323878 \\
\hline 5.086396141 & 5.038684063 & 5.040074415 & 5.03796388 & 5.006235849 & 5.190762561 & 5.076063525 & 5.039104169 & 5.037603789 & 5.053887912 \\
\hline 9.995934701 & 9.998495349 & $\mathbf{9 . 9 4 2 5 1 1 1 7 7}$ & 9.993133992 & 9.935329359 & 9.952553719 & 9.996104744 & 9.868152353 & 9.974469267 & 9.975649566 \\
\hline 10.18235786 & 10.18501406 & 10.12694084 & 10.17945265 & 10.11949104 & 10.13735812 & 10.18253425 & 10.04980732 & 10.16009145 & 10.16131579 \\
\hline
\end{tabular}

A Simulation Study on A Uniform Ditribution Over the Interval $(5,10)$ (continued)

\begin{tabular}{|c|c|c|c|c|c|c|c|c|c|}
\hline Sample 51 & Sample 52 & Sample 53 & Sample 54 & Sample 55 & Sample 56 & Sample 57 & Sample 58 & Sample 59 & Sample 60 \\
\hline 6.18441 & 9.77813 & 8.39031 & 9.65477 & 5.86795 & 8.38988 & 6.12309 & 8.57511 & 7.95065 & 5.16081 \\
\hline 7.52938 & 8.11911 & 5.41477 & 5.11005 & 8.66089 & 8.90848 & 7.80516 & 8.0271 & 8.08311 & 9.85174 \\
\hline 5.7298 & 7.77614 & 5.75094 & 7.25955 & 8.35071 & 6.34104 & 7.45682 & 8.94139 & 7.65799 & 8.71809 \\
\hline 5.65407 & 6.21417 & 7.48042 & 9.61853 & 6.2657 & 5.50628 & 5.15738 & 8.23405 & 5.0672 & 7.01167 \\
\hline 6.5132 & 7.78595 & 9.72915 & 9.85962 & 9.95137 & 7.0434 & 5.32205 & 6.69915 & 9.42174 & 7.80554 \\
\hline 9.15284 & 8.32317 & 7.41974 & 6.26129 & 8.59548 & 8.90681 & 8.82022 & 5.16808 & 5.96558 & 6.9585 \\
\hline 6.89479 & 6.23736 & 7.28764 & 8.06862 & 7.65048 & 8.65787 & 7.43745 & 8.99241 & 5.19813 & 7.17669 \\
\hline 6.18415 & 5.26976 & 5.31304 & 8.36516 & 7.54241 & 8.16157 & 8.14659 & 5.7749 & 5.26771 & 5.08625 \\
\hline 5.09403 & 8.29187 & 5.5948 & 6.40877 & 6.96558 & 9.45925 & 7.6921 & 7.50975 & 9.2622 & 9.73171 \\
\hline 5.33161 & 8.53094 & 8.40545 & 7.06276 & 8.58653 & 8.99499 & 8.0419 & 8.66307 & 7.2801 & 7.30383 \\
\hline 9.7353 & 8.62911 & 6.89256 & 8.69667 & 6.03224 & 6.58653 & 9.93124 & 5.46704 & 9.76989 & 8.63773 \\
\hline 6.15844 & 9.44765 & 9.463 & 8.25846 & 6.04034 & 7.40368 & 6.68005 & 5.10564 & 6.69407 & 6.60476 \\
\hline 8.6694 & 8.23546 & 7.00588 & 9.23245 & 7.61833 & 5.96345 & 6.88131 & 7.93937 & 7.49138 & 9.76985 \\
\hline 5.11945 & 7.36746 & 6.33881 & 9.8266 & 8.858 & 9.70288 & 6.76729 & 9.0635 & 9.56054 & 7.13157 \\
\hline
\end{tabular}




\begin{tabular}{|c|c|c|c|c|c|c|c|c|c|}
\hline 6.23517 & 8.16178 & 7.25906 & 6.74165 & 9.75388 & 6.12064 & 5.58344 & 6.87522 & 7.58093 & 8.05827 \\
\hline 7.459 & 9.85782 & 5.42105 & 7.91471 & 5.29669 & 7.81313 & 5.53362 & 6.29903 & 7.54815 & 5.36101 \\
\hline 6.15282 & 6.12379 & 8.10728 & 9.70583 & 9.26756 & 6.41615 & 9.18958 & 7.03666 & 7.1987 & 5.93602 \\
\hline 9.04239 & 7.09456 & 6.04791 & 5.88338 & 9.59331 & 6.60862 & 6.76693 & 5.99125 & 6.27664 & 7.29312 \\
\hline 9.03538 & 9.69882 & 9.82677 & 5.72132 & 9.03176 & 9.57339 & 6.97337 & 7.90027 & 7.77838 & 7.97781 \\
\hline 7.6511 & 8.3023 & 8.18709 & 6.88171 & 5.95244 & 9.5369 & 5.28247 & 6.55677 & 7.28901 & 8.93189 \\
\hline 9.09275 & 7.85247 & 9.18903 & 5.85384 & 5.91196 & 8.43459 & 6.64867 & 9.75891 & 6.79576 & 5.64979 \\
\hline 9.75152 & 9.62568 & 8.47905 & 7.79395 & 9.8703 & 8.12302 & 8.84026 & 8.61556 & 5.74694 & 8.86874 \\
\hline 9.68698 & 9.98707 & 7.59699 & 6.1366 & 8.92272 & 6.54508 & 6.90207 & 7.0073 & 5.50345 & 7.58442 \\
\hline 9.05751 & 5.42718 & 7.37152 & 8.79267 & 6.81113 & 7.50593 & 8.33312 & 9.12354 & 7.27304 & 7.31634 \\
\hline 8.91537 & 6.86456 & 8.01292 & 7.35785 & 7.20574 & 8.8904 & 5.75667 & 5.57835 & 8.59046 & 7.94288 \\
\hline 9.97589 & 9.21938 & 9.62568 & 6.36609 & 6.08722 & 7.97244 & 5.71647 & 6.68945 & 6.11918 & 6.63354 \\
\hline 8.27349 & 9.7458 & 5.75795 & 7.79634 & 7.13689 & 8.4753 & 7.53441 & 5.6029 & 8.30421 & 5.18881 \\
\hline 9.71917 & 5.41594 & 9.31332 & 8.58671 & 6.83474 & 8.97424 & 9.89658 & 6.8172 & 8.73106 & 9.23008 \\
\hline 7.60242 & 8.57215 & 9.75298 & 7.68112 & 7.80213 & 7.05231 & 7.74282 & 6.40577 & 9.84369 & 5.15754 \\
\hline 5.68316 & 5.83958 & 5.47859 & 6.92632 & 5.58554 & 5.19551 & 7.44682 & 5.19873 & 8.94484 & 9.31304 \\
\hline 9.71791 & 7.00554 & 8.3692 & 8.45708 & 7.74591 & 6.45849 & 9.84977 & 6.91658 & 9.29768 & 9.33689 \\
\hline 9.46993 & 5.51622 & 8.4123 & 9.65682 & 8.2902 & 6.75682 & 7.54487 & 7.99848 & 8.70995 & 6.43359 \\
\hline 7.20811 & 6.91121 & 5.49088 & 8.82913 & 9.56287 & 7.67468 & 5.95003 & 5.31318 & 6.39004 & 9.40255 \\
\hline 7.47061 & 5.69742 & 9.38112 & 8.34972 & 7.04619 & 6.63872 & 9.08162 & 6.81247 & 9.54834 & 8.34652 \\
\hline 9.60131 & 8.62992 & 8.85575 & 9.7735 & 9.68311 & 8.02502 & 9.32612 & 8.00922 & 7.39809 & 5.01968 \\
\hline 8.03263 & 7.5373 & 7.12965 & 6.4923 & 8.59243 & 6.24727 & 7.88883 & 5.11067 & 8.45522 & 8.00539 \\
\hline 8.25582 & 5.46626 & 8.6622 & 5.62291 & 6.38463 & 9.26127 & 8.33275 & 7.99774 & 7.79466 & 8.03671 \\
\hline 9.25829 & 8.41607 & 9.73467 & 7.69665 & 7.40422 & 7.86851 & 9.63212 & 6.09523 & 6.48612 & 7.50582 \\
\hline 9.37412 & 7.437 & 8.5678 & 8.45972 & 7.98068 & 5.0729 & 8.47921 & 5.10394 & 7.90408 & 5.00043 \\
\hline 8.67304 & 6.79355 & 7.89504 & 6.77702 & 7.6832 & 9.479 & 7.09153 & 5.27925 & 8.31455 & 8.14435 \\
\hline 6.91964 & 9.50101 & 9.64066 & 8.56392 & 9.18037 & 6.60794 & 9.17862 & 7.56436 & 9.43766 & 8.46242 \\
\hline 5.44453 & 9.21792 & 9.83241 & 8.5164 & 6.45945 & 6.89076 & 5.71788 & 8.09432 & 6.92333 & 8.59856 \\
\hline 9.65478 & 5.55052 & 9.23615 & 8.99227 & 8.73246 & 9.51937 & 7.25563 & 9.00115 & 6.81002 & 5.37905 \\
\hline 8.48216 & 9.58579 & 5.0599 & 8.42569 & 7.91049 & 5.47587 & 5.77437 & 7.82368 & 9.80094 & 7.23265 \\
\hline 7.84001 & 7.9974 & 7.89337 & 7.97349 & 9.97144 & 8.57502 & 9.02201 & 5.83154 & 9.124 & 9.02202 \\
\hline 8.02645 & 7.43669 & 8.99841 & 5.82342 & 7.19501 & 6.65335 & 7.64623 & 9.47926 & 7.8075 & 5.01488 \\
\hline 8.75322 & 9.70789 & 8.17372 & 8.88025 & 7.19618 & 9.69254 & 7.99807 & 5.4627 & 6.46246 & 9.15872 \\
\hline 8.07078 & 5.08714 & 5.98375 & 8.73024 & 8.87049 & 6.2613 & 9.58124 & 8.68369 & 7.81575 & 8.32141 \\
\hline 6.36179 & 7.86828 & 7.98099 & 5.4947 & 9.4247 & 9.97324 & 5.01158 & 9.41777 & 9.51792 & 6.86094 \\
\hline 7.01405 & 5.14188 & 9.98821 & 7.14922 & 7.63093 & 7.99576 & 9.5162 & 8.58829 & 9.70551 & 5.38763 \\
\hline 8.73944 & 6.71643 & 9.54226 & 7.91017 & 6.23884 & 8.38594 & 5.28207 & 7.3914 & 9.91407 & 5.34603 \\
\hline 6.68406 & 9.69955 & 9.07839 & 6.20943 & 8.43589 & 8.60686 & 8.63083 & 5.0309 & 6.33025 & 7.08278 \\
\hline 6.73343 & 5.52304 & 5.09884 & 6.18011 & 9.73911 & 9.54162 & 5.58246 & 7.42872 & 8.92022 & 7.4024 \\
\hline 8.13158 & 8.06549 & 9.81985 & 5.53223 & 6.35963 & 6.95308 & 6.91183 & 7.69949 & 8.84785 & 9.97761 \\
\hline 9.36368 & 9.57943 & 7.61263 & 8.48922 & 5.90058 & 9.02667 & 9.49098 & 8.6505 & 6.27251 & 9.92686 \\
\hline 9.1309 & 5.43246 & 5.14575 & 5.94565 & 8.13965 & 5.32671 & 6.5524 & 5.95253 & 9.57085 & 9.51143 \\
\hline 9.0303 & 9.8424 & 8.99437 & 5.88844 & 9.00089 & 5.33275 & 8.37706 & 9.71518 & 5.21969 & 6.87416 \\
\hline 8.45743 & 8.78069 & 7.37784 & 6.54968 & 5.81084 & 7.72496 & 5.30164 & 7.65904 & 8.38205 & 8.47167 \\
\hline 8.63807 & 7.21463 & 7.46972 & 7.21539 & 8.74623 & 7.28296 & 9.98552 & 7.16415 & 9.75178 & 5.55264 \\
\hline 7.79227 & 5.4264 & 9.90541 & 8.2935 & 5.33786 & 9.49702 & 5.22564 & 8.38841 & 7.50006 & 9.59656 \\
\hline 6.05257 & 9.60174 & 9.95031 & 9.3927 & 7.29248 & 5.68345 & 5.41758 & 6.73481 & 5.14385 & 9.47486 \\
\hline 7.94833 & 6.82022 & 9.76159 & 6.24078 & 6.71651 & 9.72959 & 9.75539 & 8.28903 & 9.93662 & 8.88633 \\
\hline 6.11134 & 5.43339 & 9.56345 & 5.74677 & 6.45726 & 9.8596 & 9.23968 & 5.89602 & 7.43733 & 8.05441 \\
\hline 6.07547 & 5.69045 & 6.67957 & 9.81997 & 9.18793 & 8.37938 & 7.93922 & 8.69046 & 9.55115 & 6.03941 \\
\hline 7.91549 & 8.96779 & 6.30639 & 5.58669 & 9.4096 & 8.4019 & 7.34804 & 8.62317 & 7.18481 & 7.02732 \\
\hline
\end{tabular}




\begin{tabular}{|c|c|c|c|c|c|c|c|c|c|}
\hline 8.06379 & 8.24225 & 6.75746 & 9.62221 & 7.84568 & 7.45376 & 5.34074 & 6.12272 & 5.98859 & 5.00399 \\
\hline 7.84529 & 8.52141 & 8.80603 & 7.27211 & 6.58523 & 6.54437 & 6.46756 & 6.22431 & 5.79068 & 6.99358 \\
\hline 6.45022 & 7.44071 & 7.13344 & 6.08751 & 8.90721 & 5.83905 & 7.00844 & 6.18728 & 8.2809 & 8.33084 \\
\hline 8.52234 & 6.98186 & 9.83986 & 7.46406 & 6.08123 & 8.96479 & 9.59466 & 8.76763 & 9.35811 & 7.36208 \\
\hline 5.88729 & 6.89956 & 5.58168 & 6.9496 & 9.26844 & 9.2954 & 5.26689 & 5.00364 & 9.74425 & 9.12353 \\
\hline 5.77397 & 9.0892 & 8.8798 & 8.21308 & 9.85811 & 5.23356 & 8.7956 & 7.17591 & 9.33207 & 8.2156 \\
\hline 5.69758 & 9.89164 & 9.35386 & 8.88636 & 7.32812 & 6.38681 & 9.9186 & 7.68886 & 5.53153 & 7.41705 \\
\hline 9.08985 & 6.41588 & 7.70854 & 9.24809 & 5.74499 & 9.13703 & 7.18093 & 9.963 & 6.58589 & 8.65935 \\
\hline 9.94847 & 6.33626 & 6.05126 & 5.84515 & 5.81283 & 6.49815 & 6.20016 & 7.44447 & 8.21266 & 8.8851 \\
\hline 7.10877 & 5.2211 & 8.27271 & 5.7172 & 8.44278 & 5.49426 & 6.826 & 8.35574 & 7.15198 & 5.04134 \\
\hline 6.7451 & 6.52118 & 8.5777 & 7.07207 & 8.44876 & 7.01084 & 6.52394 & 6.06689 & 8.91099 & 6.2181 \\
\hline 5.60918 & 7.01379 & 7.90532 & 7.68582 & 9.75914 & 5.82628 & 6.52274 & 7.42524 & 5.45111 & 6.97763 \\
\hline 5.93603 & 9.35532 & 7.96868 & 8.10172 & 8.51139 & 6.48598 & 7.02645 & 8.40771 & 7.29297 & 7.1425 \\
\hline 5.9479 & 6.24595 & 6.3582 & 6.35956 & 9.0359 & 6.77507 & 5.47117 & 6.32987 & 7.15242 & 8.19139 \\
\hline 8.49392 & 8.35634 & 8.64854 & 8.74886 & 6.90622 & 5.44751 & 7.06064 & 5.26536 & 5.91695 & 9.73825 \\
\hline 9.0644 & 5.97243 & 9.50727 & 8.08037 & 8.4277 & 6.43048 & 9.13293 & 5.75442 & 6.0637 & 7.92024 \\
\hline 9.17538 & 7.7193 & 7.7696 & 9.60945 & 9.48412 & 7.72512 & 8.02189 & 9.31978 & 6.69851 & 6.98321 \\
\hline 7.26528 & 9.90616 & 5.2074 & 5.73383 & 8.96302 & 5.51179 & 9.9519 & 5.31713 & 6.59807 & 6.25088 \\
\hline 9.89833 & 9.70411 & 6.02907 & 6.56174 & 5.80383 & 5.76764 & 8.04739 & 9.70067 & 6.24077 & 6.38908 \\
\hline 6.07587 & 9.48139 & 7.22251 & 7.48862 & 9.28034 & 8.53885 & 9.82253 & 5.00517 & 7.68063 & 5.04631 \\
\hline 7.48619 & 6.67631 & 8.52172 & 8.45061 & 7.29266 & 9.69726 & 6.91933 & 8.20324 & 7.24522 & 8.52147 \\
\hline 9.41638 & 6.76598 & 5.67395 & 5.11093 & 5.56395 & 6.71959 & 8.03859 & 6.72585 & 7.71852 & 6.0629 \\
\hline 5.03674 & 7.97037 & 8.47232 & 8.68307 & 5.32326 & 6.87318 & 5.51448 & 5.98817 & 6.55812 & 8.39528 \\
\hline 6.17799 & 5.50626 & 6.83114 & 5.39618 & 6.46491 & 5.90433 & 8.43892 & 7.13247 & 9.12011 & 6.48164 \\
\hline 8.73003 & 8.24729 & 6.55087 & 8.86766 & 6.88535 & 7.25075 & 9.8524 & 6.44582 & 5.80534 & 8.23346 \\
\hline 6.07742 & 9.83916 & 8.36661 & 5.69918 & 7.70954 & 5.83577 & 7.51845 & 8.33377 & 9.22778 & 9.99889 \\
\hline 6.13614 & 9.85828 & 5.34886 & 7.74584 & 7.00925 & 8.65152 & 7.2892 & 5.44521 & 9.83676 & 8.69858 \\
\hline 6.99859 & 7.06494 & 5.21203 & 5.98081 & 5.99386 & 8.39643 & 7.58576 & 6.46653 & 6.00821 & 8.00733 \\
\hline 6.00444 & 5.61538 & 9.96913 & 7.2796 & 9.8521 & 9.39541 & 8.95235 & 8.94116 & 5.94177 & 6.94007 \\
\hline 8.79535 & 5.19535 & 8.30057 & 9.69229 & 7.7574 & 7.23484 & 5.5718 & 5.51957 & 5.18564 & 7.06545 \\
\hline 8.4928 & 8.593 & 8.66926 & 8.98707 & 8.25488 & 9.19378 & 6.20976 & 5.54518 & 6.39157 & 5.7834 \\
\hline 6.14125 & 6.84861 & 5.95863 & 6.53861 & 8.98047 & 7.56509 & 6.75978 & 9.15693 & 7.75378 & 8.9516 \\
\hline 5.89027 & 8.47176 & 5.69369 & 6.96692 & 7.70542 & 6.92462 & 6.33232 & 5.00285 & 7.14195 & 8.84821 \\
\hline 7.40216 & 5.35465 & 8.01998 & 9.8892 & 6.41571 & 8.75031 & 9.48606 & 9.06446 & 8.4417 & 8.79506 \\
\hline 8.65471 & 7.53784 & 7.83349 & 9.24779 & 8.98193 & 6.70062 & 8.34643 & 7.31231 & 8.06331 & 8.35888 \\
\hline 7.6375795 & 7.619553 & 7.7650743 & 7.5877174 & 7.7445401 & 7.5558432 & 7.5032576 & 7.2032412 & 7.622842 & 7.5631899 \\
\hline 5.03674 & 5.08714 & 5.0599 & 5.11005 & 5.29669 & 5.0729 & 5.01158 & 5.00285 & 5.0672 & 5.00043 \\
\hline 9.97589 & 9.98707 & 9.98821 & 9.8892 & 9.97144 & 9.97324 & 9.98552 & 9.963 & 9.93662 & 9.99889 \\
\hline 7.81614 & 7.74772 & 7.974835 & 7.691235 & 7.727725 & 7.479845 & 7.45182 & 7.17003 & 7.56454 & 7.69498 \\
\hline 7.81614 & 7.74772 & 7.974835 & 7.68347 & 7.727725 & 7.479845 & 7.45182 & 7.14831 & 7.524105 & 7.69498 \\
\hline 4.9871074 & 5.0380114 & 5.010499 & 5.0611505 & 5.2496569 & 5.023629 & 4.9616958 & 4.9528785 & 5.017872 & 4.9504343 \\
\hline 7.56489 & 8.69407 & 8.80921 & -1.1908 & 7.11544 & 7.29724 & 8.53752 & 6.263 & 3.59862 & 9.88789 \\
\hline 5.275159 & 5.239106 & 5.5301486 & 5.1754348 & 5.4890802 & 5.1116864 & 5.0065152 & 4.4064824 & 5.245684 & 5.1263798 \\
\hline 5.63228 & 5.49544 & 5.94967 & 5.36694 & 5.45545 & 4.95969 & 4.90364 & 4.29662 & 5.04821 & 5.38996 \\
\hline 5.63228 & 5.49544 & 5.94967 & 5.38247 & 5.45545 & 4.95969 & 4.90364 & 4.34006 & 5.12908 & 5.38996 \\
\hline 10.0256489 & 10.0369407 & 10.0380921 & 9.938092 & 10.0211544 & 10.0229724 & 10.0353752 & 10.01263 & 9.9859862 & 10.0488789 \\
\hline 8.71074 & 13.80114 & 11.0499 & 16.11505 & 34.96569 & 12.3629 & 6.16958 & 5.28785 & 11.7872 & 5.04343 \\
\hline 10.275159 & 10.239106 & 10.5301486 & 10.1754348 & 10.4890802 & 10.1116864 & 10.0065152 & 9.4064824 & 10.245684 & 10.1263798 \\
\hline 10.63228 & 10.49544 & 10.94967 & 10.36694 & 10.45545 & 9.95969 & 9.90364 & 9.29662 & 10.04821 & 10.38996 \\
\hline 10.63228 & 10.49544 & 10.94967 & 10.38247 & 10.45545 & 9.95969 & 9.90364 & 9.34006 & 10.12908 & 10.38996 \\
\hline 4.85023245 & 4.902526362 & 4.874262748 & 4.926297266 & 5.119950755 & 4.887751257 & 4.824126996 & 4.815068944 & 4.881837064 & 4.812558006 \\
\hline
\end{tabular}




\begin{tabular}{|c|c|c|c|c|c|c|c|c|c|}
\hline 5.035483252 & 5.085896014 & 5.058649117 & 5.108811815 & 5.295499074 & 5.071652409 & 5.010316882 & 5.001584671 & 5.065950965 & 4.999164058 \\
\hline 9.977149946 & 9.988332776 & 9.989473065 & 9.890437995 & 9.972698819 & 9.974499275 & 9.986782384 & 9.964256682 & 9.937870002 & 10.00015577 \\
\hline 10.16287216 & 10.17447227 & 10.17565511 & 10.07292455 & 10.15825494 & 10.16012258 & 10.17286403 & 10.14949778 & 10.12212648 & 10.18673644 \\
\hline
\end{tabular}

A Simulation Study on A Uniform Ditribution Over the Interval $(5,10)$ (continued)

\begin{tabular}{|c|c|c|c|c|c|c|c|c|c|}
\hline Sample 61 & Sample 62 & Sample 63 & Sample 64 & Sample 65 & Sample 66 & Sample 67 & Sample 68 & Sample 69 & Sample 70 \\
\hline 8.33386 & 8.68324 & 6.06569 & 5.71666 & 9.47765 & 9.64388 & 7.85257 & 8.53535 & 6.9447 & 7.51992 \\
\hline 5.22478 & 6.66672 & 9.24647 & 8.52855 & 7.42982 & 5.91314 & 9.79971 & 6.09779 & 9.84141 & 9.88765 \\
\hline 7.48819 & 5.68176 & 6.86125 & 6.59853 & 7.1176 & 8.90473 & 9.98461 & 8.18308 & 6.11857 & 8.85597 \\
\hline 5.5882 & 7.30471 & 6.48496 & 6.38709 & 8.08738 & 7.4811 & 6.21575 & 8.14733 & 8.65565 & 5.94725 \\
\hline 5.96085 & 7.61 & 7.11843 & 9.27331 & 5.5232 & 6.13069 & 9.88163 & 5.6077 & 9.21344 & 6.15824 \\
\hline 8.56892 & 8.28776 & 6.39237 & 8.06907 & 5.24572 & 6.37339 & 8.76857 & 8.04366 & 8.47271 & 5.91623 \\
\hline 8.97896 & 5.08579 & 5.9067 & 6.5079 & 7.25708 & 6.73493 & 5.04228 & 8.13771 & 5.64076 & 5.0382 \\
\hline 8.63581 & 7.31938 & 7.69707 & 5.39935 & 8.38331 & 8.88774 & 9.96271 & 5.64265 & 5.75171 & 9.21659 \\
\hline 8.41999 & 8.35568 & 8.50346 & 8.05188 & 5.6169 & 5.58187 & 8.41413 & 8.78208 & 9.89354 & 9.00375 \\
\hline 9.78084 & 5.67967 & 5.90973 & 6.18974 & 5.29252 & 6.17131 & 8.56936 & 8.25032 & 8.54631 & 8.04344 \\
\hline 7.66588 & 8.25381 & 7.05925 & 5.09671 & 5.25951 & 7.08656 & 5.81537 & 6.7704 & 9.90095 & 8.30653 \\
\hline 6.92399 & 6.8226 & 8.71176 & 9.89 & 8.64355 & 8.75183 & 9.91843 & 6.24668 & 8.8615 & 8.68202 \\
\hline 9.69364 & 6.14229 & 6.24036 & 7.22335 & 9.51305 & 8.18923 & 8.28014 & 6.94117 & 8.95644 & 9.05494 \\
\hline 8.07553 & 8.2379 & 6.89813 & 6.1323 & 5.20797 & 5.4124 & 8.24999 & 6.88675 & 5.75946 & 6.48923 \\
\hline 7.13678 & 7.19783 & 7.17464 & 6.53436 & 7.77828 & 8.99647 & 9.93548 & 5.47309 & 9.73718 & 7.18899 \\
\hline 5.46275 & 6.68039 & 9.61244 & 9.95482 & 6.4129 & 7.81069 & 7.59375 & 8.57916 & 5.68632 & 7.90829 \\
\hline 6.66038 & 9.71753 & 8.51766 & 8.26063 & 6.53211 & 7.87249 & 9.16573 & 6.43857 & 7.87597 & 7.84278 \\
\hline 9.62912 & 7.73406 & 9.11933 & 8.01549 & 7.97769 & 7.60608 & 5.79438 & 6.26158 & 9.68509 & 7.04703 \\
\hline 8.83282 & 9.94711 & 8.43322 & 7.78444 & 6.06722 & 7.6925 & 9.85857 & 9.88778 & 9.53893 & 5.43782 \\
\hline 7.03567 & 7.52122 & 8.1134 & 7.92752 & 5.06866 & 9.39389 & 6.54179 & 8.64031 & 7.60922 & 5.00381 \\
\hline 6.42294 & 5.56357 & 9.88218 & 5.26424 & 5.97506 & 9.10147 & 7.95108 & 7.06334 & 8.93199 & 6.85971 \\
\hline 5.98707 & 8.87558 & 5.85202 & 9.65681 & 6.80356 & 6.43161 & 8.64349 & 9.90858 & 5.11364 & 8.55038 \\
\hline 5.70708 & 9.87361 & 5.3156 & 9.31189 & 8.02122 & 6.86439 & 7.59885 & 8.81479 & 6.84802 & 7.67056 \\
\hline 7.04436 & 7.07852 & 6.29023 & 6.4295 & 8.1344 & 7.75136 & 8.06189 & 6.2419 & 6.03001 & 7.14341 \\
\hline 8.02537 & 8.66816 & 6.84858 & 8.40387 & 7.65541 & 7.20096 & 6.42981 & 7.09314 & 6.9006 & 6.04064 \\
\hline 8.33408 & 9.05791 & 7.1658 & 9.71832 & 5.9763 & 7.16651 & 9.71811 & 6.69745 & 8.735 & 7.49907 \\
\hline 9.42628 & 8.03253 & 9.34731 & 8.91699 & 5.66612 & 7.47388 & 9.6291 & 8.63777 & 5.11638 & 8.67552 \\
\hline 9.00173 & 8.99835 & 8.32397 & 5.98463 & 5.33446 & 5.86943 & 9.62623 & 9.87488 & 7.67189 & 8.22861 \\
\hline 9.05446 & 6.00548 & 8.77921 & 6.77116 & 5.99397 & 9.22114 & 5.73214 & 7.75917 & 8.59905 & 9.7921 \\
\hline 6.69387 & 9.41124 & 6.02981 & 7.00684 & 6.0964 & 8.86051 & 7.0662 & 9.13609 & 8.35805 & 5.10605 \\
\hline 7.67799 & 9.72483 & 9.95558 & 8.23076 & 6.76612 & 5.81739 & 9.38686 & 6.73117 & 5.72705 & 5.02207 \\
\hline 6.16507 & 6.0107 & 6.70852 & 7.44478 & 6.21869 & 6.8646 & 5.21042 & 8.92047 & 8.23157 & 9.92693 \\
\hline 6.58252 & 7.89609 & 5.5003 & 8.53104 & 5.69627 & 8.77941 & 7.47998 & 8.73833 & 5.95011 & 9.61862 \\
\hline 5.81891 & 8.85887 & 9.18572 & 8.81609 & 6.10179 & 5.06915 & 8.14501 & 6.62071 & 9.63842 & 7.77983 \\
\hline 6.65354 & 5.58709 & 8.74049 & 7.1361 & 7.39563 & 9.53942 & 6.69487 & 8.76193 & 8.53949 & 6.5275 \\
\hline 8.76265 & 9.31455 & 6.54088 & 7.28148 & 7.12965 & 7.73246 & 8.48712 & 5.51892 & 5.79084 & 6.75708 \\
\hline 5.33782 & 8.09744 & 6.18606 & 5.98701 & 5.07749 & 6.28199 & 7.2714 & 6.32978 & 8.89635 & 5.996 \\
\hline 6.18056 & 6.14572 & 5.53232 & 8.04446 & 9.80377 & 6.44218 & 8.50149 & 6.16586 & 5.3252 & 6.64179 \\
\hline 5.24491 & 8.81609 & 8.39977 & 9.23049 & 6.00862 & 5.34227 & 7.52978 & 9.9794 & 9.12934 & 9.57183 \\
\hline 8.12176 & 9.36103 & 7.62953 & 7.55252 & 7.50491 & 5.46081 & 5.83626 & 7.39493 & 6.97651 & 6.31754 \\
\hline 8.46138 & 5.54397 & 7.60296 & 6.51692 & 8.83076 & 6.72617 & 5.41345 & 5.35451 & 7.37735 & 9.29448 \\
\hline 7.73663 & 8.01723 & 9.6759 & 7.55959 & 5.89533 & 8.91035 & 8.85853 & 8.22519 & 9.72636 & 6.76011 \\
\hline 7.691 & 7.16922 & 9.5918 & 6.82171 & 9.23448 & 5.95217 & 5.84331 & 5.54358 & 7.73501 & 7.78302 \\
\hline 9.6719 & 9.63236 & 6.66733 & 5.16886 & 8.06586 & 9.43375 & 8.80321 & 9.69027 & 5.44459 & 8.32776 \\
\hline 5.95845 & 8.62762 & 8.8266 & 7.10326 & 6.40169 & 9.85486 & 9.02197 & 5.81885 & 7.15705 & 9.67611 \\
\hline
\end{tabular}




\begin{tabular}{|c|c|c|c|c|c|c|c|c|c|}
\hline 9.6524 & 7.98828 & 5.33652 & 8.88738 & 6.48217 & 8.15836 & 7.70143 & 9.91359 & 6.43033 & 5.59025 \\
\hline 8.57258 & 9.67486 & 5.3964 & 7.99901 & 9.44993 & 6.7269 & 5.66166 & 8.8892 & 5.9972 & 7.22957 \\
\hline 6.71769 & 7.51228 & 8.44983 & 8.91037 & 6.1252 & 9.2289 & 6.59422 & 8.22895 & 7.44405 & 5.30495 \\
\hline 7.10466 & 9.44375 & 9.23458 & 5.7317 & 6.08369 & 8.10902 & 5.56866 & 9.85803 & 8.35293 & 8.20143 \\
\hline 7.37815 & 7.97606 & 6.45987 & 9.7838 & 6.94581 & 5.47738 & 9.60638 & 5.24008 & 6.69014 & 8.3159 \\
\hline 6.89403 & 9.90241 & 9.37102 & 5.91808 & 9.29558 & 7.23408 & 8.59309 & 9.45139 & 8.45893 & 8.27796 \\
\hline 8.07724 & 5.11798 & 6.25738 & 8.99032 & 9.18478 & 9.72427 & 8.68152 & 7.43384 & 9.52185 & 5.16785 \\
\hline 9.26927 & 5.80991 & 6.73841 & 7.21946 & 5.07955 & 9.26349 & 6.64582 & 7.55995 & 6.61926 & 5.80286 \\
\hline 7.87828 & 5.86586 & 6.70286 & 9.79774 & 9.65022 & 7.41293 & 5.0511 & 6.71409 & 5.60402 & 7.60797 \\
\hline 9.08609 & 5.12224 & 7.77928 & 8.84423 & 7.32385 & 5.63107 & 5.40632 & 9.53479 & 6.0485 & 8.9394 \\
\hline 6.45729 & 8.85233 & 9.25147 & 7.11062 & 5.39416 & 6.61645 & 5.52122 & 7.56912 & 5.92528 & 5.96898 \\
\hline 9.82111 & 8.33257 & 8.00819 & 8.30246 & 9.57709 & 8.45468 & 5.06487 & 8.60241 & 6.35751 & 7.7519 \\
\hline 5.10976 & 7.49392 & 9.20066 & 7.00983 & 8.68987 & 6.56298 & 7.7176 & 7.06828 & 8.23075 & 6.63957 \\
\hline 5.56465 & 8.90742 & 5.56905 & 7.77406 & 8.05317 & 9.82249 & 6.45705 & 8.01073 & 9.04162 & 7.23699 \\
\hline 9.68031 & 7.0323 & 7.74441 & 6.99614 & 7.24063 & 9.27084 & 5.83705 & 9.13224 & 8.9959 & 7.68466 \\
\hline 9.09526 & 9.29029 & 7.70087 & 8.3534 & 5.62464 & 5.09632 & 8.94895 & 5.9616 & 9.68823 & 6.34422 \\
\hline 6.06885 & 7.20337 & 7.60429 & 7.75066 & 5.13508 & 9.11684 & 8.83786 & 5.5959 & 8.85802 & 9.76222 \\
\hline 5.6508 & 7.47264 & 6.49127 & 9.87601 & 9.02235 & 9.26011 & 7.84568 & 8.04311 & 6.4105 & 9.43895 \\
\hline 8.7526 & 8.41672 & 5.86018 & 5.39356 & 6.74129 & 6.42119 & 8.73636 & 7.9601 & 6.46076 & 8.89411 \\
\hline 6.03644 & 5.51168 & 9.45856 & 6.21985 & 5.39194 & 7.28389 & 5.43208 & 6.13526 & 5.60217 & 9.9389 \\
\hline 7.14275 & 8.02242 & 6.92747 & 8.18737 & 6.02965 & 6.11066 & 7.47058 & 8.68924 & 6.10157 & 9.09118 \\
\hline 6.62505 & 5.80263 & 7.83477 & 5.88136 & 5.77446 & 6.97625 & 6.49447 & 5.66641 & 8.75575 & 8.08966 \\
\hline 7.68236 & 7.55089 & 8.41003 & 8.4743 & 7.75783 & 9.69339 & 8.2436 & 7.72624 & 6.15137 & 7.6 \\
\hline 9.45172 & 7.32735 & 7.37356 & 6.70466 & 8.93945 & 7.59987 & 8.35586 & 6.76774 & 6.02844 & 8.19393 \\
\hline 6.80846 & 5.63651 & 9.36106 & 7.67725 & 9.67556 & 5.68883 & 5.15961 & 8.96271 & 6.7943 & 9.72937 \\
\hline 6.94879 & 6.85435 & 8.45135 & 8.32445 & 6.15589 & 7.16063 & 6.13695 & 7.86882 & 9.95457 & 6.36688 \\
\hline 8.26429 & 9.78912 & 8.92117 & 7.39889 & 6.80224 & 8.74128 & 9.40541 & 5.02507 & 6.51274 & 5.54716 \\
\hline 7.04287 & 6.50473 & 8.63012 & 7.90356 & 8.45161 & 7.76939 & 6.76933 & 6.48022 & 5.28502 & 8.65768 \\
\hline 7.39116 & 9.08849 & 9.43567 & 8.86959 & 9.91994 & 7.66516 & 7.47777 & 9.54715 & 5.75461 & 5.72837 \\
\hline 9.35261 & 7.51313 & 8.9968 & 6.17384 & 8.62934 & 7.00489 & 9.77151 & 7.08121 & 5.0327 & 7.58114 \\
\hline 8.3701 & 7.01248 & 8.26069 & 8.77181 & 5.60648 & 6.16315 & 9.77179 & 9.59441 & 5.5967 & 6.07523 \\
\hline 5.22734 & 8.57884 & 7.23122 & 7.42776 & 6.8791 & 6.07049 & 7.39752 & 8.22299 & 9.83072 & 8.35479 \\
\hline 9.88564 & 5.24448 & 8.49086 & 9.50934 & 6.52388 & 8.48588 & 8.50987 & 6.42765 & 8.33584 & 7.70413 \\
\hline 8.82171 & 6.27211 & 7.36852 & 9.26275 & 7.70492 & 8.16528 & 8.53653 & 6.5852 & 9.39095 & 6.74791 \\
\hline 7.4494 & 5.56244 & 7.28072 & 9.14924 & 8.77848 & 5.95936 & 7.40984 & 8.51579 & 7.09682 & 8.46664 \\
\hline 7.54878 & 8.99541 & 7.26423 & 5.75228 & 5.502 & 8.62439 & 8.9575 & 6.86228 & 6.76772 & 9.48803 \\
\hline 5.40644 & 8.73727 & 6.89228 & 6.90719 & 6.31984 & 7.31535 & 8.33738 & 7.66226 & 7.72646 & 7.8487 \\
\hline 5.09355 & 5.16451 & 5.73235 & 9.22466 & 7.76055 & 7.44975 & 9.55632 & 5.57597 & 9.28418 & 5.86881 \\
\hline 7.48512 & 9.85053 & 8.05566 & 9.59532 & 8.48906 & 9.73986 & 7.39477 & 8.89912 & 8.1804 & 9.76484 \\
\hline 6.95781 & 9.22177 & 9.34607 & 6.85383 & 6.28402 & 9.03032 & 5.56995 & 6.90687 & 5.06927 & 5.71306 \\
\hline 8.42643 & 5.16787 & 5.51558 & 5.72018 & 5.67606 & 6.7517 & 6.76827 & 9.87248 & 7.63406 & 5.3556 \\
\hline 7.56975 & 9.78318 & 8.04863 & 9.59108 & 9.70017 & 7.41699 & 5.6624 & 9.01987 & 9.4891 & 7.51072 \\
\hline 7.71975 & 9.21024 & 9.78256 & 5.12396 & 5.59603 & 8.501 & 5.03121 & 7.93347 & 8.5607 & 8.38311 \\
\hline 8.29868 & 6.57621 & 7.73692 & 9.94762 & 9.98483 & 8.57225 & 8.96507 & 9.5054 & 8.07876 & 9.58421 \\
\hline 6.13798 & 7.70321 & 8.81989 & 7.52177 & 6.64854 & 8.88791 & 9.69224 & 6.75112 & 8.19998 & 7.53558 \\
\hline 8.9742 & 9.79037 & 9.33454 & 6.93985 & 9.50659 & 9.18756 & 8.78541 & 5.00258 & 7.04669 & 5.47221 \\
\hline 7.82483 & 7.80348 & 7.25222 & 9.15928 & 6.09933 & 5.63777 & 9.06008 & 9.3655 & 6.91488 & 5.51542 \\
\hline 5.97426 & 6.62957 & 9.47866 & 8.8637 & 6.24502 & 8.3928 & 7.21228 & 6.65438 & 5.48703 & 7.40783 \\
\hline 7.50132 & 8.98723 & 6.62637 & 6.8612 & 8.44655 & 5.4352 & 6.83272 & 7.53405 & 8.19802 & 8.50561 \\
\hline 8.73236 & 6.57317 & 7.14614 & 8.86055 & 9.10463 & 7.65611 & 9.46024 & 7.78907 & 6.04717 & 9.76321 \\
\hline 5.59234 & 7.2335 & 7.93035 & 9.76666 & 6.554 & 9.39217 & 5.09808 & 8.90024 & 7.67306 & 6.23286 \\
\hline
\end{tabular}




\begin{tabular}{|c|c|c|c|c|c|c|c|c|c|}
\hline 6.98817 & 6.63232 & 5.9989 & 6.58405 & 5.91854 & 5.60315 & 9.11419 & 7.60936 & 7.77501 & 9.83863 \\
\hline 5.02637 & 6.44252 & 6.80486 & 7.21482 & 5.11322 & 6.00649 & 5.70567 & 7.35227 & 7.35518 & 6.06713 \\
\hline 9.84123 & 7.0811 & 7.99674 & 7.61441 & 7.64477 & 6.36329 & 9.84873 & 8.20754 & 5.66138 & 7.11614 \\
\hline 8.2317 & 7.89465 & 8.17666 & 8.26099 & 6.81355 & 7.71657 & 5.32406 & 8.2478 & 7.6208 & 8.39845 \\
\hline 7.5052207 & 7.6446216 & 7.6617351 & 7.6937927 & 7.1320721 & 7.5259049 & 7.6778164 & 7.6428931 & 7.4876963 & 7.5551826 \\
\hline 5.02637 & 5.08579 & 5.3156 & 5.09671 & 5.06866 & 5.06915 & 5.03121 & 5.00258 & 5.0327 & 5.00381 \\
\hline 9.88564 & 9.94711 & 9.95558 & 9.95482 & 9.98483 & 9.85486 & 9.98461 & 9.9794 & 9.95457 & 9.9389 \\
\hline 7.52505 & 7.656605 & 7.69897 & 7.713955 & 6.8029 & 7.47749 & 7.849125 & 7.742705 & 7.61501 & 7.639265 \\
\hline 7.494755 & 7.580445 & 7.69897 & 7.713955 & 6.78418 & 7.461815 & 7.89838 & 7.69425 & 7.62164 & 7.639265 \\
\hline 4.9766337 & 5.0366479 & 5.268756 & 5.0476771 & 5.0193466 & 5.0198415 & 4.9815221 & 4.9526058 & 4.983027 & 4.9538481 \\
\hline-1.55036 & 4.65811 & 5.51358 & 5.43682 & 8.46783 & -4.65914 & 8.44561 & 7.9194 & 5.41157 & 3.8289 \\
\hline 5.0104414 & 5.2892432 & 5.3234702 & 5.3875854 & 4.2641442 & 5.0518098 & 5.3556328 & 5.2857862 & 4.9753926 & 5.1103652 \\
\hline 4.98951 & 5.16089 & 5.39794 & 5.42791 & 3.56836 & 4.92363 & 5.79676 & 5.3885 & 5.24328 & 5.27853 \\
\hline 5.0501 & 5.31321 & 5.39794 & 5.42791 & 3.6058 & 4.95498 & 5.69825 & 5.48541 & 5.23002 & 5.27853 \\
\hline 9.9344964 & 9.9965811 & 10.0051358 & 10.0043682 & 10.0346783 & 9.9034086 & 10.0344561 & 10.029194 & 10.0041157 & 9.988289 \\
\hline 7.66337 & 13.66479 & 36.8756 & 14.76771 & 11.93466 & 11.98415 & 8.15221 & 5.26058 & 8.3027 & 5.38481 \\
\hline 10.0104414 & 10.2892432 & 10.3234702 & 10.3875854 & 9.2641442 & 10.0518098 & 10.3556328 & 10.2857862 & 9.9753926 & 10.1103652 \\
\hline 9.98951 & 10.16089 & 10.39794 & 10.42791 & 8.56836 & 9.92363 & 10.79676 & 10.3885 & 10.24328 & 10.27853 \\
\hline 10.0501 & 10.31321 & 10.39794 & 10.42791 & 8.6058 & 9.95498 & 10.69825 & 10.48541 & 10.23002 & 10.27853 \\
\hline 4.83947277 & 4.901125632 & 5.139571348 & 4.91245598 & 4.883351928 & 4.883860341 & 4.844494645 & 4.814788798 & 4.846040636 & 4.816065018 \\
\hline 5.025110627 & 5.084545672 & 5.314413863 & 5.095468437 & 5.067411335 & 5.067901459 & 5.029951852 & 5.001314603 & 5.031442229 & 5.002544914 \\
\hline 9.886877093 & 9.948362658 & 9.956834803 & 9.95607461 & 9.986092209 & 9.8560893 & 9.985872154 & 9.980660834 & 9.955824547 & 9.940150579 \\
\hline 10.06923077 & 10.13301067 & 10.14179895 & 10.1410104 & 10.1721481 & 10.03729414 & 10.17191983 & 10.16651405 & 10.140751 & 10.12449216 \\
\hline
\end{tabular}

A Simulation Study on A Uniform Ditribution Over the Interval $(5,10)$ (continued)

\begin{tabular}{|c|c|c|c|c|c|c|c|c|c|}
\hline Sample 71 & Sample 72 & Sample 73 & Sample 74 & Sample 75 & Sample 76 & Sample 77 & Sample 78 & Sample 79 & Sample 80 \\
\hline 5.29534 & 5.4051 & 7.56133 & 9.96908 & 7.53051 & 8.40439 & 9.44397 & 8.96029 & 7.45155 & 5.21829 \\
\hline 8.13335 & 8.64351 & 6.27441 & 9.64264 & 5.01363 & 9.65657 & 8.45304 & 7.76633 & 5.66789 & 8.14464 \\
\hline 8.40473 & 7.07537 & 6.75974 & 9.26804 & 9.55263 & 6.24114 & 9.85276 & 6.6641 & 5.73502 & 7.88671 \\
\hline 6.42903 & 9.7191 & 8.46917 & 5.33117 & 8.09644 & 7.67821 & 7.15306 & 9.30058 & 9.63164 & 8.03104 \\
\hline 7.35789 & 7.35105 & 6.01939 & 9.11603 & 9.53862 & 6.39918 & 9.87777 & 6.37648 & 9.99317 & 8.3246 \\
\hline 9.07897 & 7.43879 & 6.43919 & 8.78856 & 7.98232 & 6.28716 & 5.43209 & 8.01208 & 5.36995 & 6.85959 \\
\hline 5.19804 & 7.19301 & 6.06461 & 9.7701 & 8.14539 & 7.11155 & 7.20384 & 7.92299 & 9.99581 & 7.60249 \\
\hline 9.3609 & 9.69603 & 9.43531 & 9.99537 & 8.61534 & 5.23147 & 7.56725 & 7.12656 & 7.62644 & 8.53846 \\
\hline 7.32324 & 8.08261 & 8.69594 & 5.5765 & 6.54041 & 5.59485 & 7.65413 & 6.99637 & 6.86903 & 5.08915 \\
\hline 6.49733 & 9.70384 & 5.80952 & 9.18232 & 9.37328 & 6.36741 & 6.33203 & 6.42279 & 8.59711 & 6.35949 \\
\hline 8.2815 & 8.06264 & 8.25435 & 7.31431 & 6.05274 & 8.3838 & 7.62851 & 6.35988 & 6.27148 & 8.37625 \\
\hline 7.08181 & 6.73147 & 7.39243 & 5.56838 & 9.20858 & 5.42332 & 9.84077 & 6.5585 & 8.82488 & 5.43078 \\
\hline 8.22865 & 9.55817 & 8.86825 & 6.11046 & 8.70642 & 5.46827 & 8.27236 & 7.72211 & 7.35678 & 7.11766 \\
\hline 6.86189 & 9.02345 & 6.80547 & 7.11598 & 9.04168 & 9.10847 & 7.49522 & 5.0822 & 6.85479 & 5.02253 \\
\hline 9.20473 & 5.46532 & 6.4591 & 5.58171 & 8.50471 & 9.07961 & 8.12461 & 9.31261 & 8.39975 & 7.63533 \\
\hline 8.82527 & 8.59435 & 7.22584 & 7.95044 & 8.47582 & 8.88789 & 8.73111 & 7.02911 & 9.67798 & 6.35092 \\
\hline 6.24431 & 6.49082 & 7.59455 & 9.8452 & 5.98017 & 8.59638 & 7.40956 & 5.47912 & 9.96034 & 5.99075 \\
\hline 7.3967 & 8.70318 & 8.04278 & 7.32597 & 5.70607 & 5.76139 & 5.73786 & 9.07153 & 5.551 & 7.04882 \\
\hline 8.45104 & 7.43454 & 5.98673 & 8.97214 & 5.06472 & 5.72077 & 6.36085 & 7.52864 & 5.69736 & 6.36641 \\
\hline 8.29331 & 9.44632 & 5.2041 & 6.91434 & 8.56621 & 5.9415 & 8.58338 & 8.45853 & 8.31169 & 9.32085 \\
\hline 5.69956 & 6.75171 & 7.9774 & 8.9045 & 8.58249 & 6.93127 & 6.92932 & 6.96405 & 8.13372 & 5.41516 \\
\hline 5.64788 & 9.6392 & 9.73921 & 6.1765 & 6.1255 & 6.0984 & 6.74669 & 8.81287 & 7.67933 & 8.0871 \\
\hline 8.76535 & 7.59807 & 7.07306 & 9.5935 & 6.51772 & 9.22505 & 8.75101 & 9.44544 & 5.35319 & 6.24701 \\
\hline 6.17504 & 9.83485 & 9.55313 & 6.68909 & 5.90225 & 8.10396 & 6.06039 & 9.08815 & 5.63077 & 5.62519 \\
\hline 5.46677 & 5.17498 & 8.69409 & 6.84149 & 7.12229 & 5.49817 & 7.52367 & 7.18283 & 9.24113 & 5.15548 \\
\hline
\end{tabular}




\begin{tabular}{|c|c|c|c|c|c|c|c|c|c|}
\hline 9.30876 & 9.23359 & 6.7705 & 6.87347 & 5.13853 & 9.25815 & 9.84215 & 6.82382 & 6.26363 & 6.6716 \\
\hline 5.01886 & 9.18277 & 7.43306 & 6.11176 & 5.99072 & 7.22315 & 9.37119 & 8.65214 & 7.63824 & 5.00687 \\
\hline 6.78122 & 8.23928 & 5.29579 & 8.17785 & 6.02131 & 7.74553 & 5.4462 & 6.29907 & 9.74905 & 5.68926 \\
\hline 6.32199 & 8.35536 & 5.22901 & 8.50337 & 5.97678 & 9.52302 & 6.97347 & 6.65162 & 6.2988 & 7.71424 \\
\hline 9.19983 & 9.29061 & 7.81481 & 6.2644 & 7.24424 & 7.08041 & 7.07182 & 6.03148 & 5.29219 & 9.65871 \\
\hline 7.43294 & 6.77657 & 7.64186 & 5.41858 & 8.88235 & 8.45258 & 8.06013 & 5.00188 & 8.14174 & 7.36088 \\
\hline 7.24019 & 6.9096 & 6.07778 & 5.55532 & 9.49193 & 9.3221 & 6.85112 & 9.30964 & 9.79674 & 5.69013 \\
\hline 6.39516 & 9.2279 & 6.75409 & 5.0645 & 7.9859 & 9.95811 & 6.06917 & 5.41132 & 9.07126 & 9.94433 \\
\hline 9.43418 & 6.20271 & 5.08832 & 6.26543 & 7.33277 & 6.90591 & 8.80166 & 7.72543 & 9.21388 & 6.42476 \\
\hline 5.39403 & 8.01287 & 9.03566 & 9.75881 & 5.44664 & 5.04796 & 7.886 & 9.22552 & 6.01976 & 6.36335 \\
\hline 9.49724 & 9.1196 & 7.08968 & 5.16515 & 5.41742 & 6.44899 & 6.27698 & 8.03042 & 8.7927 & 8.15088 \\
\hline 6.68395 & 8.48663 & 7.25289 & 9.67279 & 6.6861 & 9.68156 & 8.70112 & 5.46472 & 8.97166 & 9.53605 \\
\hline 9.48292 & 9.56944 & 8.9656 & 5.20857 & 7.92094 & 5.85746 & 9.97093 & 7.80934 & 8.39553 & 9.02484 \\
\hline 7.69481 & 5.52114 & 9.72543 & 6.95667 & 6.36609 & 8.44411 & 9.18805 & 9.90656 & 5.4816 & 9.78365 \\
\hline 6.97065 & 5.86121 & 7.48428 & 8.96358 & 8.62657 & 5.69585 & 9.66506 & 7.44959 & 9.47504 & 7.52832 \\
\hline 7.84423 & 5.97416 & 6.38899 & 6.01195 & 6.18012 & 6.66692 & 5.72219 & 8.07083 & 8.04304 & 5.65626 \\
\hline 8.67949 & 5.83938 & 8.02349 & 8.15237 & 7.08351 & 7.65652 & 7.21907 & 8.33413 & 7.58703 & 7.63994 \\
\hline 7.59177 & 5.42143 & 6.7227 & 6.29164 & 8.29775 & 5.8229 & 5.28866 & 9.01964 & 7.797 & 7.57027 \\
\hline 5.83353 & 5.66829 & 5.75984 & 7.9705 & 9.27981 & 8.07329 & 7.90288 & 6.90913 & 7.73381 & 8.5249 \\
\hline 7.57259 & 8.71823 & 7.68973 & 5.17854 & 5.48182 & 6.29955 & 9.29068 & 5.53494 & 9.04982 & 8.44965 \\
\hline 8.72939 & 8.84012 & 5.26645 & 8.74519 & 7.58978 & 5.11911 & 6.0014 & 6.88681 & 5.60474 & 8.34064 \\
\hline 7.61519 & 6.71546 & 8.03027 & 7.77736 & 9.46543 & 5.11278 & 8.00496 & 7.18887 & 6.44058 & 7.30406 \\
\hline 6.61552 & 7.09991 & 7.25714 & 6.12316 & 5.79652 & 7.72331 & 9.40073 & 5.45892 & 7.79656 & 9.41226 \\
\hline 7.50569 & 7.62317 & 5.57397 & 9.19012 & 8.3348 & 7.68349 & 9.49536 & 5.07539 & 7.69256 & 6.05531 \\
\hline 7.40309 & 6.92016 & 5.87198 & 5.45002 & 5.2735 & 5.71202 & 9.77996 & 7.40817 & 7.93739 & 6.72863 \\
\hline 7.13899 & 5.14875 & 6.63998 & 7.23377 & 7.90643 & 8.35001 & 7.01171 & 7.63708 & 9.28511 & 9.9608 \\
\hline 7.32827 & 5.68753 & 7.11705 & 5.43795 & 6.82817 & 6.72536 & 6.93648 & 5.97862 & 5.42306 & 5.10638 \\
\hline 8.37098 & 9.23052 & 7.50225 & 9.40986 & 9.7416 & 8.24445 & 5.92922 & 5.37878 & 9.15302 & 9.84264 \\
\hline 6.26015 & 9.42748 & 7.81313 & 5.673 & 6.60734 & 9.9132 & 9.48578 & 5.75398 & 9.69997 & 7.18737 \\
\hline 7.26795 & 7.48105 & 6.27431 & 5.48448 & 6.98519 & 8.55639 & 9.54436 & 7.62911 & 6.27758 & 6.62238 \\
\hline 6.39533 & 9.02117 & 9.54595 & 5.28121 & 9.74012 & 5.34607 & 9.14299 & 7.52073 & 5.95138 & 9.0428 \\
\hline 6.94118 & 5.38779 & 9.53261 & 7.22368 & 9.09579 & 6.1336 & 9.1414 & 7.79254 & 9.20972 & 6.21807 \\
\hline 9.24588 & 6.50671 & 9.95373 & 8.23706 & 6.93321 & 6.28683 & 7.54942 & 8.97603 & 9.89806 & 6.68969 \\
\hline 7.18791 & 6.2994 & 7.84705 & 5.05981 & 9.03845 & 5.07346 & 8.01857 & 7.97768 & 7.84839 & 5.08693 \\
\hline 6.55135 & 7.19112 & 9.36113 & 7.01901 & 7.65375 & 5.23822 & 9.39849 & 9.40422 & 8.45605 & 6.53516 \\
\hline 8.92805 & 6.08762 & 5.79588 & 6.70499 & 6.46709 & 6.80786 & 7.30701 & 8.88747 & 8.72765 & 8.15458 \\
\hline 9.41864 & 5.48922 & 6.24726 & 8.41119 & 6.74134 & 5.82158 & 7.84158 & 5.4823 & 7.58557 & 6.04208 \\
\hline 9.2922 & 8.6476 & 5.40192 & 8.90916 & 6.26551 & 9.62164 & 5.32289 & 9.40668 & 9.9148 & 9.36673 \\
\hline 8.13926 & 5.44262 & 5.20668 & 5.04055 & 5.51972 & 5.19939 & 6.69828 & 7.99052 & 6.84556 & 7.19024 \\
\hline 9.84548 & 9.98231 & 7.16447 & 9.30051 & 5.64217 & 5.85639 & 9.07011 & 9.1436 & 9.50644 & 7.72295 \\
\hline 8.67036 & 8.00156 & 5.29431 & 9.26896 & 5.38636 & 9.80744 & 9.60842 & 8.81366 & 5.98002 & 9.12609 \\
\hline 8.40559 & 6.43515 & 9.5363 & 7.20404 & 8.64601 & 6.07981 & 6.25599 & 9.46481 & 5.16439 & 8.17782 \\
\hline 5.29963 & 6.6301 & 7.56778 & 5.67911 & 7.85268 & 6.98859 & 6.96277 & 6.06743 & 5.9182 & 9.13599 \\
\hline 6.76764 & 7.13432 & 5.93226 & 8.49848 & 5.07038 & 6.54919 & 6.47136 & 6.84704 & 7.86795 & 5.49791 \\
\hline 9.51112 & 5.32433 & 7.59292 & 6.52796 & 5.01114 & 6.23841 & 5.35863 & 9.75385 & 9.88559 & 8.64466 \\
\hline 5.31713 & 8.5887 & 5.52494 & 5.21867 & 6.02918 & 7.02132 & 5.16717 & 7.54588 & 5.51899 & 9.64977 \\
\hline 7.15063 & 9.75176 & 9.62152 & 6.38017 & 5.68669 & 9.83771 & 9.65791 & 9.4539 & 9.27902 & 5.33515 \\
\hline 6.3164 & 6.99577 & 6.83246 & 5.7129 & 7.62097 & 8.81163 & 5.18835 & 9.44224 & 6.30472 & 6.27389 \\
\hline 6.13585 & 5.63179 & 9.86054 & 6.56385 & 9.99129 & 8.48545 & 6.24447 & 5.67577 & 7.77488 & 6.33034 \\
\hline 6.61098 & 8.03654 & 6.97019 & 9.04619 & 6.47781 & 7.72583 & 9.63305 & 8.63675 & 5.79467 & 6.38956 \\
\hline 5.16782 & 5.54582 & 8.91361 & 8.69885 & 7.7997 & 5.67967 & 5.19931 & 9.79648 & 9.81905 & 9.76787 \\
\hline
\end{tabular}




\begin{tabular}{|c|c|c|c|c|c|c|c|c|c|}
\hline 9.16174 & 8.92778 & 8.74183 & 8.93347 & 9.36532 & 9.8992 & 8.14076 & 7.90319 & 5.38274 & 5.53606 \\
\hline 6.37182 & 6.70585 & 7.40478 & 9.83507 & 7.10131 & 7.91901 & 7.2765 & 9.69637 & 8.21908 & 9.7551 \\
\hline 6.53468 & 5.95207 & 9.07178 & 5.23307 & 7.11999 & 8.66149 & 7.31296 & 8.70332 & 5.88623 & 9.15518 \\
\hline 5.71213 & 5.64929 & 7.97101 & 6.31872 & 5.67335 & 9.56325 & 6.10776 & 8.41779 & 6.14299 & 5.76685 \\
\hline 6.46098 & 7.71468 & 9.0266 & 9.02425 & 7.4055 & 7.71833 & 7.08935 & 9.87207 & 8.7474 & 5.75401 \\
\hline 8.76856 & 9.64445 & 6.92235 & 6.83029 & 9.65275 & 5.57511 & 5.5319 & 7.38721 & 7.30692 & 5.05861 \\
\hline 6.46077 & 9.95203 & 9.81265 & 6.93899 & 8.26572 & 7.0478 & 7.88366 & 6.75284 & 7.42808 & 7.34822 \\
\hline 7.46201 & 5.16277 & 8.00212 & 6.51628 & 7.01669 & 7.30998 & 5.59729 & 6.89631 & 5.26573 & 6.3456 \\
\hline 9.26282 & 9.40311 & 6.9458 & 7.95059 & 6.9822 & 9.04668 & 7.81417 & 7.84466 & 9.63677 & 5.14765 \\
\hline 8.0931 & 6.4848 & 9.70243 & 5.27327 & 5.69362 & 5.33762 & 5.90408 & 6.80198 & 8.58794 & 5.56341 \\
\hline 7.80421 & 6.68389 & 9.01429 & 5.67524 & 6.54805 & 7.21557 & 9.28523 & 9.78314 & 5.91936 & 9.07652 \\
\hline 5.24953 & 5.36996 & 7.28766 & 7.36066 & 6.77175 & 9.23456 & 5.79048 & 8.33375 & 7.59024 & 9.95954 \\
\hline 6.19756 & 8.29288 & 6.70654 & 7.82556 & 7.38983 & 9.21878 & 9.0311 & 5.39392 & 5.21681 & 6.73822 \\
\hline 5.18959 & 6.86035 & 9.25425 & 8.17053 & 6.4326 & 7.98001 & 7.25519 & 8.93437 & 8.11309 & 9.23046 \\
\hline 7.73343 & 6.09152 & 5.17068 & 7.27204 & 5.05283 & 8.0638 & 9.66958 & 8.56582 & 5.91282 & 6.88254 \\
\hline 7.2929 & 5.96746 & 5.21514 & 7.24319 & 6.74777 & 9.21517 & 7.55252 & 7.77992 & 9.99945 & 9.04506 \\
\hline 9.89837 & 7.81718 & 7.4805 & 6.01874 & 7.59569 & 7.20281 & 7.44432 & 5.46889 & 8.58833 & 5.81268 \\
\hline 6.83321 & 5.5267 & 7.69138 & 9.66579 & 5.3794 & 9.10553 & 6.04799 & 7.19692 & 9.03809 & 6.38052 \\
\hline 9.92199 & 9.99422 & 8.23611 & 9.60179 & 6.93213 & 9.81284 & 6.03293 & 9.38214 & 7.37826 & 6.17689 \\
\hline 8.47842 & 7.83244 & 5.77491 & 9.24496 & 7.82089 & 7.04397 & 7.29091 & 8.97133 & 6.52339 & 6.90199 \\
\hline 8.05264 & 8.28855 & 6.24129 & 9.25083 & 7.08711 & 9.53095 & 6.37915 & 6.59197 & 6.66995 & 9.40555 \\
\hline 6.60906 & 5.8014 & 8.29275 & 6.61656 & 6.71756 & 5.52905 & 6.10093 & 9.26536 & 7.02692 & 5.39336 \\
\hline 6.81741 & 7.12191 & 7.66751 & 7.42123 & 6.55887 & 8.11209 & 5.1328 & 5.77956 & 8.31003 & 8.93714 \\
\hline 7.40876 & 8.64889 & 7.81112 & 6.70283 & 6.98624 & 8.79452 & 5.3233 & 5.43018 & 6.09286 & 7.15431 \\
\hline 7.4339031 & 7.4862798 & 7.4113813 & 7.3654428 & 7.2229253 & 7.4025258 & 7.5258677 & 7.6293368 & 7.6223947 & 7.2909781 \\
\hline 5.01886 & 5.14875 & 5.08832 & 5.04055 & 5.01114 & 5.04796 & 5.1328 & 5.00188 & 5.16439 & 5.00687 \\
\hline 9.92199 & 9.99422 & 9.95373 & 9.99537 & 9.99129 & 9.95811 & 9.97093 & 9.90656 & 9.99945 & 9.9608 \\
\hline 7.34308 & 7.392795 & 7.41892 & 7.21386 & 7.0501 & 7.21936 & 7.42694 & 7.72377 & 7.713185 & 7.135985 \\
\hline 7.34308 & 7.392795 & 7.398605 & 7.21386 & 7.0501 & 7.20919 & 7.42694 & 7.72377 & 7.754345 & 7.152515 \\
\hline 4.9690486 & 5.1002375 & 5.0392032 & 4.9909555 & 4.9612514 & 4.9984396 & 5.084128 & 4.9518988 & 5.1160339 & 4.9569387 \\
\hline 2.12099 & 9.41622 & 5.32673 & 9.53237 & 9.12029 & 5.76911 & 7.06393 & 0.56256 & 9.94445 & 6.0408 \\
\hline 4.8678062 & 4.9725596 & 4.8227626 & 4.7308856 & 4.4458506 & 4.8050516 & 5.0517354 & 5.2586736 & 5.2447894 & 4.5819562 \\
\hline 4.68616 & 4.78559 & 4.79721 & 4.42772 & 4.1002 & 4.41838 & 4.85388 & 5.44754 & 5.50869 & 4.30503 \\
\hline 4.68616 & 4.78559 & 4.83784 & 4.42772 & 4.1002 & 4.43872 & 4.85388 & 5.44754 & 5.42637 & 4.27197 \\
\hline 9.9712099 & 10.0441622 & 10.0032673 & 10.0453237 & 10.0412029 & 10.0076911 & 10.0206393 & 9.9556256 & 10.0494445 & 10.010408 \\
\hline 6.90486 & 20.02375 & 13.92032 & 9.09555 & 6.12514 & 9.84396 & 18.4128 & 5.18988 & 21.60339 & 5.69387 \\
\hline 9.8678062 & 9.9725596 & 9.8227626 & 9.7308856 & 9.4458506 & 9.8050516 & 10.0517354 & 10.2586736 & 10.2447894 & 9.5819562 \\
\hline 9.68616 & 9.78559 & 9.79721 & 9.42772 & 9.1002 & 9.41838 & 9.85388 & 10.44754 & 10.50869 & 9.30503 \\
\hline 9.68616 & 9.78559 & 9.83784 & 9.42772 & 9.1002 & 9.43872 & 9.85388 & 10.44754 & 10.42637 & 9.27197 \\
\hline 4.831680562 & 4.96645152 & 4.903750704 & 4.85418562 & 4.823670462 & 4.861874071 & 4.949902157 & 4.814062493 & 4.982679234 & 4.819240006 \\
\hline 5.017598725 & 5.147521615 & 5.087076313 & 5.039294217 & 5.00987677 & 5.046706093 & 5.131567576 & 5.000614426 & 5.163165575 & 5.005605689 \\
\hline 9.923236298 & 9.995484587 & $\mathbf{9 . 9 5 4 9 8 4 3 3 4}$ & 9.996634878 & 9.992553845 & 9.959365444 & 9.97218869 & 9.907802391 & 10.00071591 & 9.962056125 \\
\hline 10.10694672 & 10.18189095 & 10.13987944 & 10.18308417 & 10.17885085 & 10.14442403 & 10.15772577 & 10.0909369 & 10.18731748 & 10.14721511 \\
\hline
\end{tabular}

A Simulation Study on A Uniform Ditribution Over the Interval $(5,10)$ (continued)

\begin{tabular}{|c|c|c|c|c|c|c|c|c|c|}
\hline Sample 81 & Sample 82 & Sample 83 & Sample 84 & Sample 85 & Sample 86 & Sample 87 & Sample 88 & Sample 89 & Sample 90 \\
\hline 9.07633 & 6.22709 & 8.93142 & 5.47879 & 6.10295 & 8.43147 & 7.2103 & 6.31087 & 9.48339 & 7.79791 \\
\hline 7.46731 & 8.72773 & 5.75841 & 9.42928 & 9.83704 & 9.53598 & 8.15276 & 6.07963 & 7.78439 & 8.53862 \\
\hline 9.54953 & 6.3204 & 7.05294 & 5.08947 & 9.129 & 7.80631 & 8.99794 & 6.12574 & 6.73973 & 5.4044 \\
\hline 8.66916 & 6.54669 & 9.65735 & 8.9706 & 8.08936 & 8.84479 & 5.2176 & 8.43857 & 7.93393 & 8.05026 \\
\hline 7.51807 & 8.65697 & 7.78018 & 9.56596 & 9.241 & 6.38432 & 7.57764 & 8.03424 & 8.46759 & 5.00375 \\
\hline
\end{tabular}




\begin{tabular}{|c|c|c|c|c|c|c|c|c|c|}
\hline 9.96887 & 5.04526 & 9.53446 & 8.64266 & 7.15972 & 7.0115 & 7.83808 & 6.70294 & 9.18522 & 8.87111 \\
\hline 8.38447 & 8.38428 & 8.45902 & 9.86237 & 5.54774 & 5.17332 & 8.92935 & 5.85837 & 7.97042 & 7.83184 \\
\hline 9.38132 & 6.99789 & 9.25827 & 9.4047 & 8.60551 & 9.31576 & 6.95524 & 9.38951 & 6.30501 & 7.14173 \\
\hline 8.36909 & 5.31679 & 8.42918 & 6.30254 & 7.89914 & 8.26666 & 8.75799 & 7.99675 & 9.14121 & 8.11533 \\
\hline 7.77458 & 8.37596 & 5.97245 & 8.10285 & 7.79995 & 9.42587 & 9.51766 & 7.26872 & 9.86295 & 8.04864 \\
\hline 6.90223 & 8.95205 & 9.77229 & 7.80857 & 9.80924 & 6.70765 & 9.91338 & 7.35814 & 5.009 & 6.08199 \\
\hline 5.82569 & 9.4997 & 9.09686 & 8.74921 & 9.60492 & 5.60625 & 5.32293 & 8.13181 & 6.07887 & 7.19997 \\
\hline 7.18163 & 7.20617 & 6.23193 & 8.92224 & 9.94783 & 8.37003 & 6.53386 & 5.96027 & 8.94818 & 5.16562 \\
\hline 8.05876 & 8.4156 & 8.61153 & 6.18517 & 5.20607 & 7.26223 & 8.90202 & 7.8827 & 5.79591 & 6.77074 \\
\hline 5.87638 & 7.28407 & 7.19134 & 6.04779 & 9.52896 & 5.91006 & 9.66275 & 5.54028 & 6.20219 & 7.7689 \\
\hline 9.10323 & 8.84522 & 8.4695 & 9.078 & 8.89641 & 7.40409 & 6.57756 & 7.35032 & 9.74279 & 6.11562 \\
\hline 9.90434 & 9.42639 & 9.9566 & 9.83461 & 9.88311 & 5.71111 & 8.74113 & 5.5964 & 8.56765 & 8.07186 \\
\hline 8.5548 & 9.00009 & 8.08694 & 5.61178 & 8.26934 & 7.72908 & 7.07537 & 6.99856 & 6.9398 & 7.65009 \\
\hline 9.6689 & 9.37422 & 8.89694 & 7.5591 & 7.09802 & 5.69839 & 8.76348 & 6.16627 & 9.72531 & 8.38185 \\
\hline 9.41526 & 7.44896 & 8.10825 & 5.51063 & 9.25145 & 7.51541 & 7.30225 & 9.98795 & 5.50996 & 6.3232 \\
\hline 7.95134 & 7.79711 & 5.10071 & 6.09557 & 7.74172 & 5.94703 & 6.43373 & 6.03625 & 7.4332 & 5.91577 \\
\hline 6.38257 & 6.69303 & 8.5814 & 9.62237 & 5.10002 & 5.14785 & 9.04101 & 8.30514 & 8.19413 & 6.38583 \\
\hline 9.328 & 5.34615 & 9.44272 & 8.90035 & 7.74304 & 5.6909 & 5.44873 & 9.05099 & 9.89202 & 8.88471 \\
\hline 8.63928 & 7.43681 & 8.18744 & 5.89068 & 8.72103 & 5.17332 & 5.76046 & 8.09659 & 5.8363 & 8.34229 \\
\hline 6.36035 & 5.93942 & 6.74264 & 8.29132 & 9.87873 & 5.68508 & 6.05138 & 6.26417 & 6.53717 & 7.18014 \\
\hline 9.1778 & 7.34451 & 8.38875 & 5.70707 & 6.09284 & 8.0057 & 8.72497 & 9.57313 & 8.0811 & 6.88493 \\
\hline 7.84861 & 9.60068 & 6.06075 & 5.63802 & 9.32194 & 5.63218 & 5.61786 & 7.04585 & 5.54464 & 5.2163 \\
\hline 7.42323 & 7.14332 & 6.73482 & 7.12835 & 5.29601 & 9.64634 & 7.94475 & 7.99345 & 5.24456 & 9.67143 \\
\hline 8.38202 & 6.26412 & 9.76778 & 7.3215 & 7.91254 & 6.75369 & 9.53715 & 8.36737 & 8.36967 & 9.402 \\
\hline 8.59521 & 5.1949 & 7.71135 & 5.92932 & 6.72905 & 6.66515 & 7.30941 & 7.41085 & 7.22859 & 5.09414 \\
\hline 9.14654 & 5.70358 & 6.94673 & 7.97583 & 8.30065 & 7.25863 & 8.9871 & 6.10168 & 5.22925 & 9.04202 \\
\hline 6.48628 & 9.7911 & 8.64303 & 9.98503 & 7.96757 & 9.18777 & 7.13643 & 5.74402 & 9.18795 & 6.21718 \\
\hline 9.72725 & 6.04504 & 6.15704 & 6.06796 & 5.79659 & 8.32379 & 5.51952 & 6.67743 & 9.22809 & 6.2912 \\
\hline 9.86748 & 6.26325 & 8.65568 & 8.59173 & 5.00006 & 8.74246 & 8.50563 & 8.06583 & 6.79274 & 9.79693 \\
\hline 7.78651 & 6.64519 & 9.82113 & 5.63412 & 7.50837 & 8.60388 & 8.36815 & 7.63878 & 8.30585 & 9.00874 \\
\hline 5.33484 & 9.06685 & 7.7032 & 7.25081 & 6.83956 & 6.92026 & 6.74168 & 7.2335 & 5.54603 & 9.94135 \\
\hline 5.61297 & 7.24213 & 5.7096 & 8.694 & 7.07634 & 7.93477 & 5.71694 & 8.19622 & 6.66306 & 7.8148 \\
\hline 7.65203 & 8.21584 & 8.1421 & 5.74423 & 9.88209 & 7.33768 & 9.06258 & 9.91354 & 8.76814 & 6.50362 \\
\hline 8.54449 & 9.56977 & 5.7193 & 6.16355 & 6.8599 & 8.43264 & 8.57104 & 6.77622 & 8.88427 & 6.22265 \\
\hline 5.60707 & 9.10991 & 5.31822 & 5.22034 & 9.89144 & 5.24844 & 8.68051 & 8.13552 & 7.51829 & 7.05126 \\
\hline 8.67859 & 6.90821 & 7.81467 & 9.83428 & 5.78034 & 8.35468 & 6.40224 & 5.7264 & 9.55292 & 5.62758 \\
\hline 5.10407 & 7.28666 & 5.55307 & 7.11502 & 9.58049 & 8.44556 & 8.87585 & 7.38827 & 7.81382 & 8.15318 \\
\hline 9.75566 & 7.65528 & 9.63027 & 5.88568 & 6.24805 & 8.20787 & 5.63741 & 8.69578 & 8.71738 & 8.83658 \\
\hline 7.70969 & 9.88787 & 5.97344 & 5.23668 & 8.66446 & 5.19056 & 9.77257 & 9.38541 & 7.02226 & 6.37527 \\
\hline 8.00113 & 8.60451 & 7.56841 & 8.46417 & 6.78324 & 8.15205 & 6.46249 & 9.45046 & 5.2789 & 6.67137 \\
\hline 8.54376 & 7.26556 & 9.45059 & 7.24374 & 6.10959 & 8.01725 & 6.82612 & 6.00114 & 9.018 & 5.23073 \\
\hline 8.57918 & 8.50753 & 6.11825 & 5.04661 & 8.89777 & 9.38925 & 8.19598 & 7.53332 & 6.68263 & 8.92021 \\
\hline 9.07103 & 7.97208 & 5.77974 & 6.20954 & 9.06049 & 5.37434 & 7.77542 & 7.54264 & 6.76916 & 8.79001 \\
\hline 9.26989 & 7.73261 & 7.14549 & 6.76127 & 6.52816 & 8.37528 & 6.8716 & 5.30078 & 8.10329 & 5.84675 \\
\hline 5.56392 & 8.52953 & 8.75197 & 7.62647 & 9.63035 & 7.71954 & 5.03854 & 6.76783 & 6.69635 & 9.04063 \\
\hline 7.18519 & 8.48438 & 5.79384 & 9.12714 & 8.79869 & 8.59304 & 5.48052 & 9.83521 & 9.55592 & 5.26622 \\
\hline 8.93471 & 9.51167 & 9.86274 & 7.45957 & 7.4274 & 8.81161 & 5.75054 & 9.13476 & 7.4893 & 7.40221 \\
\hline 7.23625 & 9.13255 & 8.91991 & 9.03222 & 6.7582 & 7.68769 & 8.8713 & 5.32851 & 6.29813 & 6.11422 \\
\hline 7.30418 & 9.70645 & 7.00452 & 5.11274 & 9.93808 & 6.3142 & 7.21214 & 6.25381 & 8.79968 & 8.78798 \\
\hline 5.70224 & 7.12702 & 5.70682 & 9.64889 & 7.44847 & 6.63399 & 6.27472 & 6.68976 & 9.02834 & 7.3203 \\
\hline 7.8031 & 9.26786 & 9.00692 & 7.05711 & 5.37352 & 5.44855 & 5.96876 & 6.8745 & 8.90409 & 9.49288 \\
\hline
\end{tabular}




\begin{tabular}{|c|c|c|c|c|c|c|c|c|c|}
\hline 8.22638 & 9.99282 & 5.99494 & 7.98889 & 5.80891 & 9.84553 & 9.91278 & 9.39287 & 5.21566 & 6.76083 \\
\hline 8.98805 & 5.33242 & 6.29257 & 6.61824 & 5.03523 & 8.11681 & 9.22959 & 9.48408 & 9.17563 & 8.75787 \\
\hline 9.18522 & 7.26111 & 5.63285 & 5.38287 & 8.97046 & 9.3195 & 9.35613 & 8.79452 & 8.61452 & 9.60473 \\
\hline 5.37536 & 7.18578 & 7.1684 & 9.36659 & 7.06847 & 8.24906 & 6.87939 & 5.35755 & 7.6583 & 9.85092 \\
\hline 8.69515 & 9.27406 & 8.42417 & 6.15972 & 8.5622 & 9.09463 & 9.76368 & 5.58893 & 5.77243 & 6.57097 \\
\hline 6.18468 & 6.67535 & 8.82219 & 9.80014 & 7.28625 & 9.92803 & 7.46238 & 8.487 & 9.92058 & 8.59864 \\
\hline 9.015 & 7.41978 & 5.10039 & 9.17704 & 5.06473 & 5.11896 & 6.98448 & 8.92485 & 7.82697 & 9.20877 \\
\hline 6.23037 & 6.52546 & 7.27584 & 9.11296 & 8.22946 & 7.45643 & 7.73959 & 7.05418 & 7.0088 & 8.24658 \\
\hline 9.34539 & 8.41991 & 5.33893 & 8.64336 & 8.37211 & 7.97283 & 9.43204 & 6.66904 & 8.72435 & 7.84374 \\
\hline 8.48799 & 8.40256 & 8.45211 & 8.46259 & 5.94704 & 5.2207 & 6.55709 & 5.22487 & 9.85847 & 7.80304 \\
\hline 7.29593 & 7.37494 & 8.2561 & 6.75092 & 7.94508 & 7.91946 & 8.33038 & 7.13965 & 9.0289 & 6.9178 \\
\hline 8.67699 & 7.08657 & 7.12942 & 9.03059 & 8.87441 & 5.25413 & 6.12891 & 9.54423 & 7.47211 & 6.71345 \\
\hline 7.22994 & 7.42033 & 6.68438 & 6.61835 & 9.28037 & 7.6746 & 8.20667 & 9.26325 & 6.48228 & 6.87201 \\
\hline 7.78658 & 9.35449 & 5.25175 & 8.4785 & 6.78095 & 9.40123 & 5.56376 & 5.88447 & 7.66836 & 8.73826 \\
\hline 5.80629 & 6.34893 & 6.05232 & 7.49887 & 6.8357 & 5.75933 & 9.72439 & 8.37426 & 7.98882 & 6.40707 \\
\hline 7.30855 & 8.53711 & 6.31937 & 8.79807 & 8.26445 & 6.65658 & 6.90127 & 5.04827 & 7.39614 & 8.36559 \\
\hline 8.41501 & 5.77193 & 8.65249 & 9.96891 & 8.69938 & 6.99918 & 7.78425 & 6.39136 & 8.00812 & 6.41539 \\
\hline 5.98222 & 5.1469 & 9.71738 & 5.93387 & 5.10037 & 5.12661 & 8.92279 & 8.63533 & 8.98703 & 9.44222 \\
\hline 8.18762 & 7.95081 & 8.33455 & 7.31054 & 7.23103 & 8.19221 & 5.45183 & 7.4644 & 7.19731 & 9.62582 \\
\hline 9.21356 & 7.90997 & 9.68697 & 9.06071 & 9.2253 & 6.41638 & 5.1527 & 7.31179 & 8.54084 & 5.32475 \\
\hline 7.0458 & 6.62475 & 7.03171 & 6.55648 & 7.14657 & 6.08043 & 8.42496 & 6.84821 & 9.4327 & 5.7495 \\
\hline 5.5853 & 5.4036 & 9.48263 & 8.36331 & 5.9696 & 8.73767 & 5.10864 & 7.11747 & 8.14308 & 9.81236 \\
\hline 8.12823 & 8.13646 & 8.55005 & 7.41726 & 8.02554 & 9.05935 & 8.95674 & 6.87275 & 8.39471 & 8.17648 \\
\hline 9.56013 & 8.10491 & 8.64901 & 8.69259 & 7.8158 & 7.57775 & 9.75613 & 7.33403 & 5.03069 & 5.27356 \\
\hline 6.77433 & 7.00529 & 9.77188 & 9.04303 & 6.7092 & 7.16666 & 7.45141 & 5.79434 & 7.29223 & 5.45911 \\
\hline 8.12497 & 5.61858 & 7.8484 & 9.57103 & 5.62544 & 6.58559 & 6.34629 & 7.48521 & 8.14389 & 9.36212 \\
\hline 8.60444 & 5.44335 & 7.1199 & 6.53212 & 5.50608 & 5.19065 & 6.37115 & 6.97049 & 8.85598 & 8.77562 \\
\hline 7.61063 & 7.29401 & 7.30566 & 7.65378 & 8.95417 & 8.50668 & 6.69215 & 5.037 & 8.87605 & 5.01347 \\
\hline 5.39954 & 8.69618 & 7.3053 & 9.25626 & 7.27645 & 8.8889 & 5.82267 & 9.86805 & 8.90112 & 8.66835 \\
\hline 9.42613 & 7.43183 & 7.10642 & 7.62359 & 6.76323 & 9.17422 & 6.24667 & 5.59745 & 5.62909 & 9.16497 \\
\hline 9.57625 & 9.58287 & 5.35302 & 7.1753 & 6.65114 & 5.35956 & 5.59339 & 5.4509 & 6.65904 & 8.12805 \\
\hline 9.95597 & 7.2754 & 8.39801 & 5.11721 & 7.33697 & 8.85338 & 8.58702 & 5.04665 & 5.6132 & 8.20784 \\
\hline 7.31365 & 8.58626 & 9.68817 & 5.57323 & 5.95172 & 9.25482 & 8.73844 & 5.93622 & 6.93194 & 8.23233 \\
\hline 5.24302 & 9.94828 & 6.47925 & 5.73817 & 7.96237 & 8.05379 & 6.69024 & 9.32954 & 7.22746 & 9.64555 \\
\hline 9.96533 & 7.12839 & 7.67128 & 7.38048 & 9.1061 & 7.37199 & 9.51538 & 5.92175 & 6.49251 & 8.13069 \\
\hline 7.34558 & 8.00808 & 6.31561 & 5.76741 & 6.90026 & 5.02683 & 9.65726 & 9.8701 & 6.62672 & 9.11493 \\
\hline 6.71969 & 6.36953 & 9.81843 & 8.09429 & 8.34533 & 8.98084 & 5.5026 & 8.20076 & 8.83492 & 9.18321 \\
\hline 5.4686 & 9.56864 & 6.76206 & 5.09924 & 7.9987 & 6.67188 & 5.23925 & 8.71601 & 6.2656 & 9.31354 \\
\hline 7.41439 & 9.87928 & 5.30882 & 8.61303 & 7.39773 & 9.78339 & 9.65924 & 6.22194 & 9.36869 & 5.01302 \\
\hline 9.00601 & 5.08797 & 9.66356 & 8.11113 & 8.22486 & 7.66119 & 5.12186 & 9.0265 & 7.63114 & 8.17037 \\
\hline 5.44717 & 8.8959 & 6.73766 & 5.52687 & 5.91976 & 8.06607 & 9.04503 & 8.5356 & 6.61399 & 7.3298 \\
\hline 5.30261 & 8.55193 & 9.20313 & 8.29471 & 9.38746 & 6.28996 & 9.92739 & 5.42419 & 5.12521 & 5.42682 \\
\hline 9.4693 & 6.03858 & 7.75878 & 6.44164 & 7.99109 & 9.06713 & 8.60666 & 7.29492 & 5.51489 & 6.56011 \\
\hline 7.01584 & 8.98609 & 9.00099 & 7.42043 & 6.81574 & 7.5904 & 5.18286 & 8.72836 & 7.54818 & 8.53305 \\
\hline 7.840856 & 7.691014 & 7.6778775 & 7.5009392 & 7.6395676 & 7.4588462 & 7.5225205 & 7.3637649 & 7.6330737 & 7.5759014 \\
\hline 5.10407 & 5.04526 & 5.10039 & 5.04661 & 5.00006 & 5.02683 & 5.03854 & 5.037 & 5.009 & 5.00375 \\
\hline 9.96887 & 9.99282 & 9.9566 & 9.98503 & 9.94783 & 9.92803 & 9.92739 & 9.98795 & 9.92058 & 9.94135 \\
\hline 8.029945 & 7.55212 & 7.76948 & 7.47922 & 7.771495 & 7.681145 & 7.456895 & 7.303355 & 7.726375 & 7.82332 \\
\hline 8.029945 & 7.55212 & 7.735065 & 7.528985 & 7.807875 & 7.681145 & 7.52001 & 7.303355 & 7.726375 & 7.82332 \\
\hline 5.0551107 & 4.9957126 & 5.0513939 & 4.9970761 & 4.9500606 & 4.9770983 & 4.9889254 & 4.98737 & 4.95909 & 4.9537875 \\
\hline 6.85587 & 9.27482 & 5.6166 & 8.48803 & 4.73083 & 2.73103 & 2.66639 & 8.78295 & 1.97858 & 4.07635 \\
\hline
\end{tabular}




\begin{tabular}{|c|c|c|c|c|c|c|c|c|c|}
\hline 5.681712 & 5.382028 & 5.355755 & 5.0018784 & 5.2791352 & 4.9176924 & 5.045041 & 4.7275298 & 5.2661474 & 5.1518028 \\
\hline 6.05989 & 5.10424 & 5.47013 & 5.05797 & 5.61575 & 5.36229 & 5.04002 & 4.60671 & 5.45275 & 5.64664 \\
\hline 6.05989 & 5.10424 & 5.53896 & 4.95844 & 5.54299 & 5.36229 & 4.91379 & 4.60671 & 5.45275 & 5.64664 \\
\hline 10.0185587 & 10.0427482 & 10.006166 & 10.0348803 & 9.9973083 & 9.9773103 & 9.9766639 & 10.0378295 & 9.9697858 & 9.9907635 \\
\hline 15.51107 & 9.57126 & 15.13939 & 9.70761 & 5.00606 & 7.70983 & 8.89254 & 8.737 & 5.909 & 5.37875 \\
\hline 10.681712 & 10.382028 & 10.355755 & 10.0018784 & 10.2791352 & 9.9176924 & 10.045041 & 9.7275298 & 10.2661474 & 10.1518028 \\
\hline 11.05989 & 10.10424 & 10.47013 & 10.05797 & 10.61575 & 10.36229 & 10.04002 & 9.60671 & 10.45275 & 10.64664 \\
\hline 11.05989 & 10.10424 & 10.53896 & 9.95844 & 10.54299 & 10.36229 & 9.91379 & 9.60671 & 10.45275 & 10.64664 \\
\hline 4.920092551 & 4.859072611 & 4.916274266 & 4.860473341 & 4.812174102 & 4.839950055 & 4.852100089 & 4.85050222 & 4.821450046 & 4.816002764 \\
\hline 5.102830301 & 5.04400541 & 5.099149369 & $\mathbf{5 . 0 4 5 3 5 5 7 5 2}$ & 4.998793965 & 5.025570743 & $\mathbf{5 . 0 3 7 2 8 3 7 0 8}$ & $\mathbf{5 . 0 3 5 7 4 3 3 1 8}$ & 5.007736228 & 5.002484899 \\
\hline 9.970128168 & 9.994084232 & 9.957855061 & 9.98629226 & 9.949082841 & 9.929277827 & 9.928637665 & 9.989212999 & 9.921825941 & 9.9426012 \\
\hline 10.15558836 & 10.18043835 & 10.14285728 & 10.17235562 & 10.13375773 & 10.11321369 & 10.11254964 & 10.17538534 & 10.10548374 & 10.12703422 \\
\hline
\end{tabular}

A Simulation Study on A Uniform Ditribution Over the Interval $(5,10)$ (continued)

\begin{tabular}{|c|c|c|c|c|c|}
\hline Sample 91 & Sample 92 & Sample 93 & Sample 94 & Sample 95 & Sample 96 \\
\hline 5.86572 & 9.13582 & 9.92336 & 9.02606 & 8.32079 & 8.35625 \\
\hline 9.04363 & 5.6464 & 8.95248 & 6.20083 & 7.81803 & 5.15256 \\
\hline 8.66985 & 9.32944 & 9.54133 & 6.31972 & 6.33105 & 5.18741 \\
\hline 8.65893 & 6.23357 & 6.21029 & 6.07032 & 5.156 & 5.24429 \\
\hline 7.31984 & 9.96533 & 7.1128 & 6.08748 & 9.92731 & 8.64478 \\
\hline 6.13777 & 7.84763 & 8.37369 & 8.29732 & 5.56943 & 7.89334 \\
\hline 5.10856 & 6.2574 & 9.3278 & 9.06103 & 6.48706 & 5.16776 \\
\hline 8.3243 & 5.34829 & 6.04274 & 8.5992 & 8.47424 & 7.36028 \\
\hline 9.69419 & 6.37833 & 8.50027 & 8.37377 & 9.53429 & 8.6986 \\
\hline 7.33633 & 6.98037 & 8.411 & 6.71552 & 6.46917 & 7.80143 \\
\hline 8.43794 & 6.00619 & 6.10002 & 7.45449 & 5.59396 & 8.04843 \\
\hline 8.26051 & 7.41828 & 7.8871 & 9.89547 & 9.74559 & 6.92853 \\
\hline 8.35463 & 6.14928 & 9.62454 & 8.61974 & 8.01633 & 6.35648 \\
\hline 8.83131 & 5.92827 & 5.42501 & 8.09416 & 7.94183 & 8.87483 \\
\hline 8.47461 & 7.24523 & 7.95075 & 8.72966 & 6.82337 & 9.20996 \\
\hline 8.45167 & 9.26223 & 9.42486 & 8.51323 & 7.862 & 8.08856 \\
\hline 7.9084 & 6.13401 & 8.1216 & 8.31812 & 7.25036 & 8.75127 \\
\hline 7.50068 & 5.69459 & 6.18948 & 8.40196 & 8.81836 & 8.11296 \\
\hline 7.51844 & 8.6257 & 8.21662 & 7.221 & 8.87436 & 8.12392 \\
\hline 7.48881 & 5.28283 & 6.86155 & 5.97045 & 5.96877 & 8.68363 \\
\hline 8.9915 & 5.6318 & 6.04671 & 6.83479 & 6.12325 & 5.8468 \\
\hline 8.93084 & 6.88242 & 7.93402 & 6.53323 & 9.82366 & 8.93326 \\
\hline 6.7716 & 9.90217 & 9.31641 & 9.50011 & 5.39753 & 8.28776 \\
\hline 5.93379 & 7.26842 & 5.81641 & 6.92787 & 8.29695 & 8.69209 \\
\hline 8.10334 & 8.95291 & 7.82231 & 9.47038 & 7.45759 & 8.75926 \\
\hline 9.91705 & 8.11124 & 5.57305 & 7.79777 & 8.39773 & 6.67366 \\
\hline 9.18388 & 6.17943 & 5.67291 & 9.40558 & 8.84358 & 9.78651 \\
\hline 8.35432 & 5.15284 & 5.50311 & 5.44203 & 5.72602 & 6.91125 \\
\hline 7.51931 & 8.72996 & 8.43469 & 9.3203 & 9.64756 & 6.87958 \\
\hline 5.68985 & 7.14541 & 8.00684 & 5.64337 & 6.88585 & 5.84195 \\
\hline 9.04888 & 9.5941 & 7.00703 & 9.90737 & 8.348 & 8.11501 \\
\hline 7.33399 & 8.23909 & 5.56518 & 5.54059 & 6.08967 & 9.52512 \\
\hline 7.96985 & 7.04011 & 6.82435 & 5.59351 & 5.43279 & 7.42408 \\
\hline 9.26438 & 7.30276 & 9.85036 & 6.61649 & 7.64762 & 5.70815 \\
\hline 6.89913 & 8.74842 & 8.37378 & 8.67246 & 9.49837 & 7.95319 \\
\hline 7.65159 & 6.20507 & 9.23769 & 5.91164 & 6.26615 & 5.2339 \\
\hline
\end{tabular}




\begin{tabular}{|c|c|c|c|c|c|}
\hline 9.71752 & 7.43167 & 8.39145 & 5.43253 & 6.73935 & 9.32309 \\
\hline 8.15593 & 8.19124 & 6.00996 & 5.46631 & 7.32051 & 6.62743 \\
\hline 7.78713 & 5.17834 & 5.15968 & 8.07533 & 6.37032 & 9.47532 \\
\hline 7.84396 & 7.27345 & 9.77918 & 6.82975 & 5.95845 & 6.16939 \\
\hline 6.1413 & 7.46559 & 8.38298 & 5.74795 & 5.58061 & 5.88661 \\
\hline 8.81012 & 7.18406 & 9.68431 & 5.49727 & 6.05596 & 5.70989 \\
\hline 9.74056 & 9.09032 & 5.07699 & 8.9039 & 8.03717 & 9.37978 \\
\hline 7.5583 & 8.27282 & 8.92244 & 6.36074 & 7.30441 & 6.36012 \\
\hline 9.4902 & 6.5142 & 9.78872 & 6.9561 & 7.2033 & 8.63422 \\
\hline 6.60529 & 6.66159 & 6.96212 & 9.4697 & 7.3725 & 6.55171 \\
\hline 5.87705 & 7.892 & 5.33919 & 6.51344 & 6.46308 & 6.25676 \\
\hline 6.08031 & 8.28815 & 9.11105 & 8.2719 & 7.1645 & 9.85544 \\
\hline 7.84844 & 5.47504 & 6.16136 & 8.93055 & 8.52803 & 8.57674 \\
\hline 9.09614 & 8.07122 & 7.14447 & 9.2174 & 9.72313 & 9.20112 \\
\hline 7.70841 & 5.21424 & 9.35447 & 8.0174 & 9.65122 & 9.69076 \\
\hline 6.51518 & 7.69133 & 6.51391 & 8.00974 & 7.16047 & 8.55198 \\
\hline 5.07027 & 8.65295 & 5.1765 & 9.47664 & 7.93638 & 6.73614 \\
\hline 6.64277 & 7.02979 & 9.97077 & 7.6124 & 6.19742 & 8.39844 \\
\hline 8.44305 & 6.01492 & 8.14348 & 7.66167 & 5.29104 & 8.80795 \\
\hline 6.97692 & 8.53164 & 8.61289 & 8.8483 & 6.38396 & 6.81859 \\
\hline 5.5855 & 8.89811 & 8.58016 & 7.25241 & 9.33989 & 6.56283 \\
\hline 8.82062 & 5.1131 & 8.16859 & 5.63221 & 5.27245 & 7.67655 \\
\hline 8.33591 & 5.12164 & 7.05508 & 6.44184 & 7.02534 & 7.88164 \\
\hline 9.2979 & 8.68988 & 9.27228 & 9.34844 & 9.335 & 7.57438 \\
\hline 9.71805 & 6.04889 & 7.72917 & 5.06334 & 5.31684 & 8.79569 \\
\hline 7.6126 & 7.75411 & 5.57882 & 7.00005 & 8.99788 & 7.25569 \\
\hline 9.65659 & 7.94325 & 5.49581 & 6.0681 & 7.17909 & 5.34649 \\
\hline 6.71937 & 9.28436 & 6.81028 & 6.18412 & 8.20189 & 9.34453 \\
\hline 5.71262 & 9.02465 & 6.57436 & 8.25482 & 9.17856 & 8.40376 \\
\hline 8.39577 & 7.25704 & 8.6201 & 5.9777 & 7.34209 & 8.45199 \\
\hline 7.39871 & 5.14884 & 9.28114 & 7.78681 & 6.5373 & 5.00843 \\
\hline 5.22456 & 8.58504 & 9.6408 & 9.27244 & 8.08614 & 7.96656 \\
\hline 8.89719 & 8.81921 & 7.58059 & 5.10885 & 5.35376 & 6.98271 \\
\hline 6.84458 & 8.92073 & 6.55887 & 8.27278 & 5.29776 & 7.29433 \\
\hline 5.92116 & 8.37494 & 7.85216 & 9.68456 & 7.49348 & 8.25419 \\
\hline 8.56835 & 8.01447 & 5.30782 & 7.6975 & 9.61352 & 6.63628 \\
\hline 5.91579 & 9.57159 & 7.9622 & 9.60373 & 8.84222 & 6.83221 \\
\hline 5.10213 & 9.26724 & 9.67652 & 8.55332 & 5.24183 & 5.74419 \\
\hline 7.02156 & 8.08377 & 7.17982 & 6.10646 & 7.33902 & 6.95325 \\
\hline 6.21123 & 5.67665 & 5.05615 & 5.5194 & 7.92216 & 8.10212 \\
\hline 6.71114 & 9.27757 & 7.30917 & 6.88397 & 8.96757 & 9.10216 \\
\hline 6.36315 & 9.13549 & 7.71975 & 6.47669 & 6.02399 & 8.84095 \\
\hline 6.95813 & 9.89787 & 9.53642 & 8.83447 & 8.47992 & 9.13579 \\
\hline 5.10689 & 5.73173 & 5.97953 & 6.50463 & 6.33939 & 6.58964 \\
\hline 5.67011 & 9.23312 & 6.90265 & 8.1055 & 7.59999 & 5.02374 \\
\hline 8.53045 & 7.50885 & 5.77513 & 9.95609 & 5.09864 & 8.37032 \\
\hline 5.08424 & 5.09385 & 7.02799 & 7.33499 & 6.40422 & 7.95074 \\
\hline 9.75538 & 9.40583 & 7.72641 & 7.47409 & 5.49103 & 7.84894 \\
\hline 7.4949 & 7.93969 & 7.07998 & 7.16771 & 6.36073 & 8.6891 \\
\hline 6.43358 & 6.798 & 7.11137 & 6.3902 & 7.81645 & 9.46907 \\
\hline 9.31756 & 9.75418 & 7.67978 & 8.21611 & 6.76287 & 7.81748 \\
\hline
\end{tabular}




\begin{tabular}{|c|c|c|c|c|c|}
\hline 5.73522 & 8.71442 & 7.82624 & 8.38104 & 7.01808 & 8.26516 \\
\hline 6.53467 & 6.85715 & 8.85128 & 9.32542 & 7.40197 & 7.84465 \\
\hline 5.74598 & 8.78821 & 5.21513 & 5.60309 & 6.2175 & 8.49354 \\
\hline 8.22455 & 5.90085 & 8.03437 & 5.88735 & 9.68126 & 8.26842 \\
\hline 9.68162 & 8.91189 & 7.49925 & 6.36036 & 8.17312 & 5.74127 \\
\hline 6.30008 & 6.55256 & 9.84196 & 8.16357 & 5.97884 & 6.61848 \\
\hline 6.12873 & 8.92951 & 9.34479 & 5.07719 & 6.94334 & 5.1727 \\
\hline 7.07208 & 9.06227 & 7.6745 & 9.3218 & 5.45887 & 6.86871 \\
\hline 8.8666 & 7.43472 & 8.8224 & 9.27425 & 9.21156 & 6.6648 \\
\hline 5.14106 & 7.59071 & 5.66978 & 6.69994 & 6.65606 & 7.02232 \\
\hline 5.92786 & 9.75104 & 8.55321 & 8.47856 & 8.93543 & 6.29558 \\
\hline 7.92396 & 5.0605 & 8.51211 & 6.12383 & 8.32792 & 6.86106 \\
\hline 5.46176 & 5.96106 & 5.46517 & 5.55743 & 5.25943 & 6.62341 \\
\hline 7.5018816 & 7.5296848 & 7.6000715 & 7.488132 & 7.3283083 & 7.5491417 \\
\hline 5.07027 & 5.0605 & 5.05615 & 5.06334 & 5.09864 & 5.00843 \\
\hline 9.91705 & 9.96533 & 9.97077 & 9.95609 & 9.92731 & 9.85544 \\
\hline 7.538805 & 7.54978 & 7.77574 & 7.543245 & 7.277385 & 7.846795 \\
\hline 7.58545 & 7.54978 & 7.77574 & 7.543245 & 7.277385 & 7.846795 \\
\hline 5.0209727 & 5.011105 & 5.0067115 & 5.0139734 & 5.0496264 & 4.9585143 \\
\hline 1.62205 & 6.49833 & 7.04777 & 5.56509 & 2.65831 & -4.60056 \\
\hline 5.0037632 & 5.0593696 & 5.200143 & 4.976264 & 4.6566166 & 5.0982834 \\
\hline 5.1709 & 5.09956 & 5.55148 & 5.08649 & 4.55477 & 5.69359 \\
\hline 5.07761 & 5.09956 & 5.55148 & 5.08649 & 4.55477 & 5.69359 \\
\hline 9.9662205 & 10.0149833 & 10.0204777 & 10.0056509 & 9.9765831 & 9.9039944 \\
\hline 12.09727 & 11.1105 & 10.67115 & 11.39734 & 14.96264 & 5.85143 \\
\hline 10.0037632 & 10.0593696 & 10.200143 & 9.976264 & 9.6566166 & 10.0982834 \\
\hline 10.1709 & 10.09956 & 10.55148 & 10.08649 & 9.55477 & 10.69359 \\
\hline 10.07761 & 10.09956 & 10.55148 & 10.08649 & 9.55477 & 10.69359 \\
\hline 4.885022428 & 4.874885294 & 4.870371831 & 4.877832015 & 4.914458505 & 4.820858627 \\
\hline 5.069021743 & 5.059249269 & 5.054898167 & 5.062089988 & 5.097398926 & 5.007166084 \\
\hline 9.918295047 & 9.966587272 & 9.972028649 & 9.957344932 & 9.928557645 & 9.856669446 \\
\hline 10.10182109 & 10.15191534 & 10.15755976 & 10.14232812 & 10.11246663 & 10.03789593 \\
\hline
\end{tabular}

A Simulation Study on A Uniform Ditribution Over the Interval $(5,10)$ (continued)

\begin{tabular}{|c|c|c|c|}
\hline Sample 97 & Sample 98 & Sample 99 & Sample 100 \\
\hline 6.06981 & 7.196 & 6.26284 & 7.8831 \\
\hline 9.32288 & 6.3471 & 9.21827 & 8.70313 \\
\hline 7.48206 & 5.5979 & 5.66504 & 9.28785 \\
\hline 5.55559 & 8.9968 & 9.67986 & 5.68158 \\
\hline 6.947 & 7.4866 & 6.61298 & 5.95971 \\
\hline 5.91696 & 6.8486 & 6.75368 & 7.04876 \\
\hline 9.33183 & 5.4834 & 7.73034 & 9.27037 \\
\hline 6.13787 & 8.7877 & 8.30374 & 6.8351 \\
\hline 7.91341 & 7.3681 & 5.10907 & 8.98629 \\
\hline 5.61384 & 5.5579 & 7.51722 & 9.66273 \\
\hline 9.87432 & 5.1294 & 8.15625 & 8.05645 \\
\hline 9.92302 & 9.1291 & 8.57707 & 8.20657 \\
\hline 6.03787 & 7.27 & 9.22589 & 7.6988 \\
\hline 9.72749 & 6.2725 & 5.20106 & 6.38067 \\
\hline 8.09217 & 8.2146 & 7.54133 & 9.60089 \\
\hline 6.23279 & 7.2335 & 5.3949 & 7.06617 \\
\hline
\end{tabular}




\begin{tabular}{|c|c|c|c|}
\hline 8.43305 & 7.0493 & 7.31186 & 5.0671 \\
\hline 5.49295 & 8.5185 & 6.88068 & 7.80063 \\
\hline 8.56085 & 6.4932 & 9.53571 & 5.31537 \\
\hline 7.27365 & 6.4598 & 6.97175 & 5.88511 \\
\hline 6.90708 & 5.8214 & 7.61343 & 6.67453 \\
\hline 7.18443 & 7.6224 & 5.0642 & 9.90066 \\
\hline 9.4264 & 6.9188 & 9.54913 & 5.46897 \\
\hline 6.0879 & 6.3237 & 6.29712 & 7.24598 \\
\hline 5.66977 & 9.6747 & 9.38268 & 5.17785 \\
\hline 7.12944 & 6.8755 & 9.1996 & 8.46317 \\
\hline 5.70038 & 7.1885 & 7.23291 & 9.3592 \\
\hline 7.82778 & 5.7789 & 8.92291 & 7.85412 \\
\hline 7.29484 & 5.0599 & 9.63473 & 6.73056 \\
\hline 7.62466 & 6.9333 & 6.36501 & 9.20233 \\
\hline 5.83423 & 5.7463 & 5.39357 & 5.70454 \\
\hline 8.25272 & 5.2885 & 5.99405 & 5.09388 \\
\hline 5.51224 & 5.6281 & 9.57646 & 9.42176 \\
\hline 7.39638 & 5.9825 & 9.99017 & 7.65081 \\
\hline 8.83294 & 6.6391 & 7.7901 & 5.14826 \\
\hline 5.28796 & 8.035 & 9.48686 & 5.03304 \\
\hline 8.17813 & 9.0869 & 7.56432 & 8.14471 \\
\hline 7.64573 & 8.536 & 6.88738 & 8.72843 \\
\hline 7.70506 & 9.42 & 7.02482 & 6.23009 \\
\hline 6.16992 & 6.6216 & 8.55453 & 9.32639 \\
\hline 7.57258 & 8.2968 & 5.28664 & 7.84153 \\
\hline 8.7078 & 5.9088 & 5.65771 & 7.28903 \\
\hline 8.33025 & 9.3744 & 5.87207 & 5.27575 \\
\hline 5.72844 & 6.2502 & 6.4284 & 7.56508 \\
\hline 6.32623 & 7.8262 & 6.0977 & 8.174 \\
\hline 8.79575 & 9.3025 & 6.42639 & 7.77993 \\
\hline 7.9307 & 9.7544 & 9.99122 & 8.17368 \\
\hline 8.35479 & 7.9114 & 5.19999 & 6.1531 \\
\hline 5.90778 & 8.7225 & 7.94356 & 7.13526 \\
\hline 8.04123 & 8.9966 & 9.27531 & 5.6579 \\
\hline 7.37065 & 9.0767 & 8.66941 & 9.92382 \\
\hline 5.54969 & 9.6716 & 7.19476 & 7.44799 \\
\hline 7.85046 & 8.0562 & 6.08785 & 5.15208 \\
\hline 9.62641 & 7.4477 & 9.78405 & 8.02122 \\
\hline 7.68202 & 7.1621 & 9.04268 & 8.50513 \\
\hline 7.51337 & 7.3528 & 5.80391 & 6.21122 \\
\hline 5.69447 & 8.8631 & 6.96021 & 9.57822 \\
\hline 5.86206 & 9.6208 & 8.87941 & 6.69874 \\
\hline 6.61621 & 5.9569 & 5.5584 & 9.50193 \\
\hline 6.65144 & 8.4801 & 8.25181 & 6.95405 \\
\hline 8.36578 & 9.7359 & 8.23355 & 6.22647 \\
\hline 8.09951 & 5.133 & 7.71347 & 9.06748 \\
\hline 6.13185 & 7.0257 & 6.93728 & 7.48149 \\
\hline 5.87743 & 8.9391 & 8.12423 & 5.65091 \\
\hline 7.30891 & 9.3774 & 5.58766 & 6.79316 \\
\hline 7.10725 & 7.654 & 6.47934 & 9.08146 \\
\hline 9.83079 & 9.5668 & 6.35068 & 5.83882 \\
\hline
\end{tabular}




\begin{tabular}{|c|c|c|c|c|c|}
\hline 6.92512 & 6.6621 & 5.00661 & 9.82196 & & \\
\hline 9.11534 & 8.4111 & 8.17905 & 6.12704 & & \\
\hline 8.18818 & 9.0767 & 8.79637 & 8.13823 & & \\
\hline 6.48844 & 5.6448 & 7.49101 & 9.54837 & & \\
\hline 9.70725 & 5.3183 & 7.1652 & 7.82948 & & \\
\hline 9.6157 & 7.207 & 7.50207 & 9.77303 & & \\
\hline 6.259 & 8.6083 & 6.00227 & 7.87994 & & \\
\hline 8.22683 & 6.7875 & 6.34944 & 6.07921 & & \\
\hline 6.72646 & 6.0566 & 9.87433 & 7.84758 & & \\
\hline 5.47933 & 7.7005 & 6.65184 & 9.38024 & & \\
\hline 5.82503 & 8.0103 & 6.45383 & 5.42378 & & \\
\hline 9.36158 & 8.145 & 5.14453 & 9.9326 & & \\
\hline 9.37292 & 8.0809 & 6.31914 & 6.50298 & & \\
\hline 9.81604 & 7.4179 & 5.37785 & 5.25003 & & \\
\hline 5.98888 & 7.0782 & 7.88375 & 6.43828 & & \\
\hline 8.08137 & 5.4684 & 7.20134 & 7.69912 & & \\
\hline 7.16463 & 5.9356 & 6.76466 & 5.2247 & & \\
\hline 7.40421 & 5.7293 & 6.96203 & 6.99122 & & \\
\hline 8.51 & 8.3009 & 6.53925 & 9.4795 & & \\
\hline 6.22441 & 6.515 & 9.75635 & 8.72266 & & \\
\hline 8.6553 & 5.2525 & 6.88367 & 8.73144 & & \\
\hline 5.08693 & 5.9486 & 8.11424 & 5.93893 & & \\
\hline 6.74125 & 9.9999 & 7.10754 & 7.14105 & & \\
\hline 5.42597 & 6.3915 & 8.75378 & 5.44901 & & \\
\hline 7.10294 & 5.6972 & 8.27718 & 7.25408 & & \\
\hline 5.74783 & 8.5311 & 5.83878 & 8.9644 & & \\
\hline 6.35642 & 5.0193 & 9.85545 & 7.90506 & & \\
\hline 9.47188 & 5.0379 & 6.45491 & 6.85422 & & \\
\hline 5.25334 & 8.4727 & 7.26275 & 8.52135 & & \\
\hline 8.91317 & 5.0021 & 8.39853 & 5.36629 & & \\
\hline 7.91003 & 5.0652 & 9.01336 & 9.01209 & & \\
\hline 7.77594 & 6.9555 & 8.0859 & 9.74762 & & \\
\hline 9.78677 & 9.9591 & 7.20513 & 9.88968 & Mean & Var \\
\hline 7.3914974 & 7.315718 & 7.4168335 & 7.5100028 & 7.502282583 & 0.021270717 \\
\hline 5.08693 & 5.0021 & 5.00661 & 5.03304 & 5.0536892 & 0.003032216 \\
\hline 9.92302 & 9.9999 & 9.99122 & 9.9326 & 9.9556945 & 0.001421328 \\
\hline 7.383515 & 7.2015 & 7.21902 & 7.674805 & 7.5061214 & 0.06102876 \\
\hline 7.383515 & 7.19775 & 7.24783 & 7.607945 & 7.50175925 & 0.063832164 \\
\hline 5.0377993 & 4.952121 & 4.9566761 & 4.9833704 & 5.004226092 & 0.003093163 \\
\hline 2.22502 & 9.9899 & 9.11322 & 3.1926 & 5.5251445 & 14.49896702 \\
\hline 4.7829948 & 4.631436 & 4.833667 & 5.0200056 & 5.004565166 & 0.085082868 \\
\hline 4.76703 & 4.3955 & 4.49566 & 5.21589 & 5.0035185 & 0.255328655 \\
\hline 4.76703 & 4.403 & 4.43804 & 5.34961 & 5.0122428 & 0.244115039 \\
\hline 9.9722502 & 10.049899 & 10.0411322 & 9.981926 & 10.00525145 & $\mathbf{0 . 0 0 1 4 4 9 8 9 7}$ \\
\hline 13.77993 & 5.2121 & 5.66761 & 8.33704 & 10.4226092 & 30.93163269 \\
\hline 9.7829948 & 9.631436 & 9.833667 & 10.0200056 & 10.00456517 & 0.085082868 \\
\hline 9.76703 & 9.3955 & 9.49566 & 10.21589 & 10.0035185 & 0.255328655 \\
\hline 9.76703 & 9.403 & 9.43804 & 10.34961 & 10.0122428 & 0.244115039 \\
\hline 4.902308471 & 4.81429076 & 4.818970236 & 4.846393412 & & \\
\hline 5.085685961 & 5.000834481 & 5.005345623 & 5.031782316 & & \\
\hline 9.924266558 & 10.00116603 & 9.992483827 & 9.933848984 & & \\
\hline
\end{tabular}




\begin{tabular}{|l|l|l|l|l|l|}
\hline $\mathbf{1 0 . 1 0 8 0 1 5 4 3}$ & $\mathbf{1 0 . 1 8 7 7 8 4 3 9}$ & $\mathbf{1 0 . 1 7 8 7 7 8 2 2}$ & $\mathbf{1 0 . 1 1 7 9 5 5 4 2}$ & & \\
\hline
\end{tabular}

LCL=Lower Confidence Limit; UCL=Upper Confidence Limit; LB=Lower Bound ; UB= Upper Bound

\begin{tabular}{|c|c|c|}
\cline { 2 - 3 } \multicolumn{1}{c|}{} & Mean & Var \\
\hline Mean & 7.502282583 & 0.021270717 \\
\hline Man & 5.0536892 & 0.003032216 \\
\hline$M($ even $)$ & 9.9556945 & 0.001421328 \\
\hline$M($ odd $)$ & 7.5061214 & 0.06102876 \\
\hline$T 1$ & 7.50175925 & 0.063832164 \\
\hline$T 2$ & 5.004226092 & 0.003093163 \\
\hline$T 3$ & 5.5251445 & 14.49896702 \\
\hline$T 4$ & 5.004565166 & 0.085082868 \\
\hline$T 5$ & 5.0035185 & 0.255328655 \\
\hline$W 1$ & 5.0122428 & 0.244115039 \\
\hline$W 2$ & 10.4226092 & 30.93163269 \\
\hline$W 3$ & 10.00525145 & 0.001449897 \\
\hline$W 4$ & 10.00456517 & 0.085082868 \\
\hline$W 5$ & 10.0035185 & 0.255328655 \\
\hline & 10.0122428 & 0.244115039 \\
\hline
\end{tabular}

\section{REFERENCES}

[1] Balakrishnan, N. and Cohen, A. C. Order Statistics and Inference. New York: Academic Press, 1991.

[2] Balakrishnan, N. and Rao, C. R. (Eds.). Handbook of Statistics, Vol. 16: Order Statistics: Theory and Methods. Amsterdam, Netherlands: Elsevier, 1998.

[3] Balakrishnan, N. and Rao, C. R. (Eds.). Order Statistics: Applications. Amsterdam, Netherlands: Elsevier, 1998.

[4] David, H. A. Order Statistics, 2nd ed. New York: Wiley, 1981.

[5] Gibbons, J. D. and Chakraborti, S. (Eds.). Nonparametric Statistic Inference, 3rd ed. exp. rev. New York: Dekker, 1992.

[6] Hogg, R. V. and Craig, A. T. Introduction to Mathematical Statistics, 3rd ed. New York: Macmillan, 1970.

[7] Rose, C. and Smith, M. D. Order Statistics. New York: Springer-Verlag, pp. 311-322, 2002.

[8] Rose, C. and Smith, M. D. "Computational Order Statistics." Mathematica J". 9, 790-802, 2005.

[9] Arnold B. C., Balakrishnan N., and Nagaraja, H. N.(1992). A First Course in Order Statistics. John Wiley, New York.

[10] Coles, S. (2001). An Introduction to Statistical Modeling of Extreme Values. Springer Verlag, London.
[11] David H. A. and Nagaraja, H. N. (2003). Order Statistics, Third Edition. John Wiley, New York.

[12] Pickands, J. (1975). "Statistical inference using extreme order statistics". Annals of Statistics 3, 119-131.

[13] Bairamov, I and Parsi, S. (2011) "Order statistics from mixed exchangeable random variables." Journal of Computational and Applied Mathematics, 35, Issue 16, 4629-4638.

[14] Bairamov I (2010), Order Statistics. In: Penelope Peterson, Eva Baker, Barry McGaw, (Editors), International Encyclopedia of Education, Volume 7, pp. 360-365. Oxford: Elsevier.

[15] Bairamov, I. and Tanil, H. (2008) "Distributions of exceedances of generalized order statistics." Statistics, 42, Issue $1,67,76$.

[16] Bairamov, I. and Khan, M.K.(2007) "On exceedances of record and order statistics." Proceedings of the American Mathematical Society. Volume 135, Number 6, pp. 1935-1945.

[17] Bayramoglu, I. "Ordered Random Variables - Recent Developments," 14th International Congress on Computational and Applied Mathematics, Anakara University, Middle East Technical University, Izmir University of Economics, Turkish Central Bank, Antalya, Sept. 29- Oct. 2 , 2009.

[18] Bairamov, I. "Waiting times for exceedances based on order statistics", Conference on Ordered Statistical Data and Its Applications, RWTH Aachen University - Aachen, Germany, 7-8 March 2008 